\title{
The evaluation of normal and pathological bladder sensations
}

Citation for published version (APA):

Heeringa, R. (2012). The evaluation of normal and pathological bladder sensations. [Doctoral Thesis, Maastricht University]. Universitaire Pers Maastricht. https://doi.org/10.26481/dis.20121101rh

Document status and date:

Published: 01/01/2012

DOI:

10.26481/dis.20121101rh

Document Version:

Publisher's PDF, also known as Version of record

\section{Please check the document version of this publication:}

- A submitted manuscript is the version of the article upon submission and before peer-review. There can be important differences between the submitted version and the official published version of record.

People interested in the research are advised to contact the author for the final version of the publication, or visit the DOI to the publisher's website.

- The final author version and the galley proof are versions of the publication after peer review.

- The final published version features the final layout of the paper including the volume, issue and page numbers.

Link to publication

\footnotetext{
General rights rights.

- You may freely distribute the URL identifying the publication in the public portal. please follow below link for the End User Agreement:

www.umlib.nl/taverne-license

Take down policy

If you believe that this document breaches copyright please contact us at:

repository@maastrichtuniversity.nl

providing details and we will investigate your claim.
}

Copyright and moral rights for the publications made accessible in the public portal are retained by the authors and/or other copyright owners and it is a condition of accessing publications that users recognise and abide by the legal requirements associated with these

- Users may download and print one copy of any publication from the public portal for the purpose of private study or research.

- You may not further distribute the material or use it for any profit-making activity or commercial gain

If the publication is distributed under the terms of Article $25 \mathrm{fa}$ of the Dutch Copyright Act, indicated by the "Taverne" license above, 


\section{The Evaluation of Normal and Pathological Bladder Sensations}

Rhea Heeringa 
Drs. R. Heeringa

Maastricht 2012

The Evaluation of Normal and Pathological Bladder Sensations

Cover design: R. Heeringa and M.A.C. Smits

Lay-out: R. Heeringa

Production: Datawyse | Universitaire Pers Maastricht

ISBN: 9789461591784

Copyright $@ 2012$ R. Heeringa, Maastricht 


\title{
The Evaluation of Normal and Pathological Bladder Sensations
}

\author{
PROEFSCHRIFT
}

ter verkrijging van de graad van doctor

aan de Universiteit Maastricht,

op gezag van de Rector Magnificus, prof. dr. L.L.G. Soete

volgens het besluit van het College van Decanen,

in het openbaar te verdedigen

op donderdag 1 november 2012 om 10:00 uur

door

Rhea Heeringa

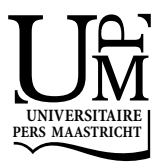




\section{Promotor:}

Prof. dr. Ph.E.V.A. van Kerrebroeck

Prof. dr. S.G.G. de Wachter; Universiteit Antwerpen

\section{Copromotor:}

Dr. G.A. van Koeveringe

\section{Beoordelingscommissie:}

Prof. dr. H. Steinbusch (voorzitter)

Prof. dr. R.A. de Bie

Dr. J.P.F.A. Heesakkers; Universitair Medisch Centrum Nijmegen

Prof. dr. J.G. Nijhuis

Prof. dr. J.J. Wyndaele; Universiteit Antwerpen

The production of this thesis was financially sponsored by: Astellas Pharma BV, Eurocept BV, Fast Guide BV, Ipsen Farmaceutica BV, Pfizer BV, WAMU (Wetenschappelijke Activiteiten Maastrichtse Urologie), Zambon Nederland B.V. 


\section{Contents}

$\begin{array}{lll}\text { Chapter } 1 & \text { Introduction }\end{array}$

$\begin{array}{lll}\text { Chapter } 2 & \text { On the nature of bladder sensation: } & 17\end{array}$

the concept of sensory modulation

Chapter $3 \quad$ Degree of urge, perception of bladder fullness 33

and bladder volume: how are they related?

Chapter $4 \quad$ "Focused introspection" during naturally increased 45

diuresis: description and repeatability of a new

method to study bladder sensation non-invasively

Chapter $5 \quad$ Normal bladder sensations in healthy volunteers:

a focus group investigation

Chapter $6 \quad$ Do patients with OAB experience bladder sensations in the same way as healthy volunteers?

A focus group investigation

Chapter $7 \quad$ General discussion

Chapter $8 \quad$ Summary / Samenvatting 89

$\begin{array}{lll}\text { Chapter } 9 & \text { CV \& publications - presentations } & 97\end{array}$

$\begin{array}{lll}\text { Chapter } 10 & \text { Acknowledgments / Dankwoord } & 101\end{array}$ 


$\begin{array}{ll}\text { List of abbreviations } \\ \text { CDV } & \text { Compelling Desire to Void } \\ \text { CNS } & \text { Central Nervous System } \\ \text { DO } & \text { Detrusor Overactivity } \\ \text { ICC } & \text { Intraclass correlation coefficient } \\ \text { ICS } & \text { International Continence Society } \\ \text { OAB } & \text { Overactive Bladder Syndrome } \\ \text { PPIUS } & \text { Patient's Perception of Intensity of Urgency Scale } \\ \text { SD } & \text { Standard Deviation } \\ \text { SR-BD } & \text { Sensation Related - Bladder Diary } \\ \text { VAS } & \text { Visual Analogue Scale }\end{array}$


Introduction 


\section{Introduction}

Individuals without lower urinary tract symptoms are not constantly bothered by what is happening in their bladder. They can assess their bladder sensations at any time (introspection) and can act upon these sensations if they have an absolute need to void or if they choose to do so (behaviour). However, there are individuals for whom the normal pattern of bladder sensations, introspection and behaviour, is altered. One of the pathological conditions in which patients experience altered bladder sensations is the overactive bladder syndrome (OAB). The aetiology of $O A B$ is still largely unknown and unfortunately current treatment options do not relieve symptoms in all cases. The history and assessment of patient complaints is an important tool in diagnosing $\mathrm{OAB}$, however, we do not have a clear and unambiguous terminology describing normal and pathological bladder sensations. This makes it difficult for patients to describe their symptoms and difficult for the clinicians to understand and correctly diagnose the problem. Having a clear terminology is therefore important in order to further unravel the aetiology of pathological conditions and to prevent miscommunication between doctor and patient.

Furthermore, it is essential that bladder sensations can be studied by means of a noninvasive technique. Diagnostic methods should allow doctors to evaluate the entire pattern of bladder sensations ranging from no sensation to an absolute need to void. Only then we can compare sensations experienced at home with sensations experienced during the diagnostic phase in the hospital. Unfortunately, the current diagnostic tools are either invasive (urodynamics) or do not describe the entire pattern in which an absolute need to void develops (sensation related bladder diaries).

\section{Normal bladder sensation}

Individuals without lower urinary tract symptoms are capable of defining how much urine is in their bladder and can act upon this sensation accordingly. Without a fear of leakage, voiding can be postponed until there is a toilet available and the situation is suitable to void. The purpose of studying normal bladder sensations is to provide information about normal conscious bladder control. Understanding these normal bladder sensations will help us to unravel the aetiology of lower urinary tract dysfunction.

By means of cystometry Denny-Brown et al. ${ }^{1}$ and Nathan ${ }^{2}$ were the first to study normal sensations related to bladder filling and micturition. In 1933, Denny-Brown and Robertson described a specific pattern of bladder sensations during artificial bladder filling ${ }^{1}$. Both experienced sensations of pressure, desire, fullness and discomfort or pain but they disagreed on the character of the sensation: episodic or continuous. Later Nathan pointed out that different sensations might be originating from different anatomical locations. Sensations of awareness and desire were located in the bladder, while sensations of imminent micturition would originate from a lower location in the urinary tract, possibly the proximal urethra ${ }^{2}$.

In 1998, bladder sensations experienced by healthy volunteers during cystometry were systematically evaluated ${ }^{3}$. This study showed that there are three normal bladder sensations: a first sensation, a first desire to void and a strong desire to void ${ }^{3}$. A first sensation in the lower pelvis, described as weak and fluctuating, is probably only perceived during artificial filling. This sensation can easily be ignored for a short period of time. The first 
desire to void is described as a constant sensation in the lower abdomen, gradually becoming more intense. The strong desire to void is a constant sensation that will make an individual to go find a place to void. Finally the sensation of urge or great tension will develop. A few years later, this study was repeated in another 50 healthy volunteers and the same results were found ${ }^{4}$. The terminology mentioned above, describes the pattern in which the need to void will develop in healthy volunteers. This terminology has been endorsed by the International Continence Society (ICS) ${ }^{5}$ and is widely used in practice these days.

Abrams et al. have described the pattern in which these normal bladder sensations develop to be episodic ${ }^{6}$. The individual sensations gradually increase in intensity. As the bladder fills, the sensations appear in a standard order. The first sensation appears first, then declines and is followed by further episodic sensations with increasing intensity, namely the first desire to void and the normal desire to void. Eventually the final, strong desire to void, also called urge, triggers the subject to find a toilet to void. Although in character there are three different sensations described, the episodic pattern is not the only way to interpret how these sensations develop. Another way in which normal bladder sensations can develop is a continuous sensation model ${ }^{7}$. This model states that signals are continuously generated within the bladder. This means that there are no episodic, waxing and waning sensations but that, as the bladder fills, a continuous sensation increases in intensity and possibly character, can be probed or sampled by the mind if desired and finally transforms into a sensation of urge that consequently leads to finding a toilet.

\section{Pathological bladder sensation}

Sensations that originate from the bladder are perceived as normal or pathological depending on whether they are increased or decreased, compared to the normal situation. Sensations can be interpreted as pathological when they are increased or decreased in amplitude or frequency, occur in inappropriate situations or when they are painful. In this thesis the focus lies on increased bladder sensation generation or perception as seen in the overactive bladder syndrome.

\section{The overactive bladder}

For many people the normal pattern of bladder sensations, introspection and behaviour, is altered. One of the pathological conditions in which patients can experience altered bladder sensations is the overactive bladder syndrome (OAB). Patients with OAB complain about urgency with or without urgency incontinence and usually with frequency and nocturia ${ }^{5}$. While some patients only experience altered bladder sensations, others, can also experience normal bladder sensations, in addition to these altered sensations.

$\mathrm{OAB}$ affects almost 1 in 5 adults, induces lifestyle changes and influences the quality of life $^{8-10}$. The lifestyle changes that one may think of are: reduced fluid intake and avoidance of places where one does not know the way to the toilets. Behavioural and pharmacological treatments for $O A B$ are being used. Behavioural treatment consists of bladder training, timed voiding, fluid intake modification and exercises for the pelvic floor muscles. The combination of behavioural and pharmacological treatment is shown to be more effective for older people than one therapy alone ${ }^{11}$. Unfortunately, our current 
treatment options do not relieve the symptoms of all patients sufficiently and new treatments options are therefore still necessary.

\section{Urgency}

Urgency is the cornerstone symptom of the overactive bladder syndrome. The International Continence Society (ICS) defined this sensation as "a sudden compelling desire to void that is difficult to defer" ${ }^{\prime \prime}$. Urge is defined as a desire to void that is experienced by healthy volunteers ${ }^{6}$. It gradually increases with increasing bladder volume an can usually be deferred from the mind until at a desired location voluntary voiding is commenced ${ }^{6}$. Urgency, on the other hand, is seen as a pathological, all-or-none sensation that can clearly be differentiated from a sensation of bladder fullness ${ }^{6,12}$. Urgency is however not only seen in patients with $O A B$, but can also be experienced in other pathological conditions, as for example in urolithiasis, urinary tract infection and after bladder/pelvic radiotherapy. The above mentioned also entails that there are different causes of urgency, one of these is for example involuntary detrusor contractions (DO). To objectify these contractions, cystometry can be performed. These contractions seem to correlate with urgency and urgency incontinence ${ }^{13}$. However, patients with urgency may also have a normoactive detrusor (e.g. no DO during bladder filling) during cystometry ${ }^{14}$ and on the contrary, healthy volunteers without lower urinary tract symptoms may show DO on a cystometry ${ }^{15}$. Another possible cause of urgency can also be related to spontaneous relaxations of the urethra ${ }^{16,17}$. These relaxations have been described as urethral instability or overactivity. If one only includes the relaxations with $>20 \mathrm{cmH}_{2} \mathrm{O}$ or $3 \%$ of the maximum urethral pressure, these relaxations appear more often in patients with urgen$\mathrm{cy}^{17}$.

Fitzgerald and colleagues found that the location of the urgency sensation can be different for different subjects ${ }^{18}$. For example more than half of the patients with painful bladder syndrome and a minority of patients with $O A B$ localized their urgency to both suprapubic and vulvar/urethral locations. Healthy subjects and patients with stress-urinary incontinence, however, locate the sensation of urge always in the suprapubic region ${ }^{18}$. A possible explanation might lie in different aetiology.

Lately the idea has been put forward, that urgency might be a sensation that can be graded. Moreover, there is a discussion about whether urgency is in fact a different sensation than the normal urge to void or whether it is the same sensation but more pronounced ${ }^{19-21}$. Evidence for this can be found in literature. Blaivas and colleagues showed by means of questionnaires that there are two types of urgency ${ }^{19}$. One is an intensification of the normal urge to void and the other is a different sensation. These different types of urgency might represent different aetiology and respond differently to treatment options. This indicates that a comprehensive classification of these possible different types of urgency is of great importance.

\section{Terminology}

The ongoing debate on urge and urgency shows that we have not yet figured out the exact aetiology of OAB and that we have not clearly defined the terminology when discussing (ab)normal bladder sensations. However, in order to prevent misdiagnosis it is 
important that clinicians use clear and unambiguous terminology to describe lower urinary tract symptoms and that patients understand and also convey these terms.

Digusu and colleagues showed that most women attending urodynamic clinics in different countries do not know the correct meaning of the terminology describing lower urinary tract symptoms which is currently used by clinicians ${ }^{22}$. For example only one third of the women in the cited study, knew the correct definition of increased daytime frequency and only $39 \%$ knew the term urge urinary incontinence. This problem can lead to miscommunication, misdiagnosis and mistreatment. It is therefore important that we come up with a standardised unambiguous terminology that is clear to both clinicians and patients. Unfortunately, this is difficult because sensations are subjective and therefore hard to study and objectify.

\section{Methods to evaluate bladder filling sensations}

Bladder sensations can be evaluated using different diagnostic tools. Cystometry and sensation-related bladder diaries are tools that are currently available for this purpose.

\section{Cystometry}

During cystometry, bladder, urethral and abdominal pressures are measured by means of catheters. Because changes in bladder pressure can be due to either stress in the bladder wall (active or passive) or to changes in pressure around the bladder, it is important to measure the abdominal pressure simultaneously. The component of intravesical pressure can be calculated by subtracting the abdominal pressure from the intravesical pressure. Cystometry gives us the opportunity to study bladder sensations under controlled circumstances. However, placing a urethral catheter may induce sensations of its own, which might influence the perception of the lower urinary tract and bladder filling $^{23}$. Moreover, using artificial filling rates compared to physiological filling rates may alter afferent activity ${ }^{24}$ and perception of a full bladder ${ }^{15}$. It is therefore not surprising that if patients receive certain instructions on what they might or should perceive upon filling, that they perceive these sensations even when the bladder is not filled as showed by a study from Erdem et al. $^{25}$. However, if patients only receive a general explanation on what they might feel, only 3 out of 59 subjects report perception of bladder filling with an empty bladder ${ }^{26}$. These demonstrations clearly show the difficulty in evaluating a subjective parameter such as bladder sensation. This difficulty becomes even worse if we consider the difference in method and language that can be used by the patient or physician. Dompeyre et al. showed that the method used to evaluate bladder sensation may influence the pattern in which bladder sensation is described ${ }^{27}$. Although the ICS has accepted the three normal bladder sensations developing in an episodic manner, Dompeyre et al. showed that when a subject fills out their bladder sensation on a visual analogue scale, the development of the sensations is continuous. These studies, with sometimes contradicting results, demonstrated that not only the technique had an influence on the results, but the investigator and the instruction given to the subject had an impact on the study results as well. This and the fact that sensations are subjective, makes the evaluation of bladder sensations extremely difficult. 


\section{Bladder diaries}

Another tool to collect information on how an individual perceives bladder sensations is the use of the sensation-related bladder diary (SR-BD). A bladder diary is a noninvasive tool that can be used to evaluate lower urinary tract function in daily life ${ }^{28}$. Daytime frequency, nocturia, maximum, minimum and mean voided volumes and fluid intake can be determined ${ }^{5}$.

In order to be able to study bladder sensation in daily life during a physiological bladder filling, the sensation-related bladder diary was introduced ${ }^{29}$. Not only the volume of each void and possible incontinence was noted, but subjects were also asked to grade their degree of urge by a predefined scale ranging from "no sensation" to "need to void now". The combination of the grade of desire and the corresponding voided volume was considered a tool to evaluate bladder sensation. It was found that the mean voided volumes, at every degree of urge, were significantly different and that there was a correlation between the voided volume and the degree of urge $\mathrm{e}^{29-31}$. After the introduction of the new scale it has been used and adjusted by several authors ${ }^{32-34}$. After the modification of the degree of urge scale by others, there are now several scales in use, for example the original degree of urge scale ${ }^{29}$, the Patient's Perception of Intensity of Urgency Scale ${ }^{34}$ and the Urinary Sensation Scale ${ }^{35}$. Although all scales are quite similar, there is no uniformity and therefore studies using these different scales are difficult to compare. A standardised, validated scale used in all studies is preferable.

Besides the fact that degree of urge scale gives us the opportunity to study bladder sensations in a non-invasive manner, it only provides information on the bladder sensation experienced just before voiding. Therefore, it cannot be used to study the development of bladder sensation up until an absolute need to void. Another disadvantage of this method is that subjects are asked to relate their sensation to the predefined terms on the bladder diary.

In practice, filling out a SR-BD is difficult for some people while it is a burden to combine measuring voided volumes with their professional and social life. Having a sensory scale that correlates well with the voided volume could replace the need to measure all voided volumes and would therefore be ideal, as also proposed by others ${ }^{34}$.

Figure 1 is an example of the SR-BD that we used in our studies. 
Figure 1 shows a sensation-related bladder diary as used in our studies. This example is filled out by a healthy volunteer. In the columns from the left to the right the following information is asked for: drinking (time and volume), voiding (time and volume), incontinence, estimation of the fullness of the bladder (ranging from empty to full) and the degree of urge (ranging from "no desire to void" to "need to void now").

\begin{tabular}{|c|c|c|c|c|c|c|c|c|c|}
\hline \multirow{2}{*}{\multicolumn{2}{|c|}{$\begin{array}{l}\text { Drinken } \\
\text { Vul s.v.p. in wanneer } \\
\text { en hoeveel u drinkt }\end{array}$}} & \multicolumn{2}{|c|}{$\begin{array}{l}\text { Plassen } \\
\text { Vul S.v.p in wanneer } \\
\text { en hoeveel U plast }\end{array}$} & \multirow{2}{*}{$\begin{array}{c}\text { Ongewild } \\
\text { urine } \\
\text { verlies? } \\
D=\text { druppel } \\
S=\text { scheut } \\
S t=\text { straal } \\
P=\text { gehele } \\
\text { plas }\end{array}$} & \multirow[t]{2}{*}{$\begin{array}{l}\text { In welke mate is volgens } \mathrm{U} \text { de blaas gevuld? } \\
\text { (zet bij iedere keer dat } \mathrm{U} \text { gaat plassen, een kruisje bij hoever } \mathrm{U} \\
\text { denkt dat uw blaas gevuld is ) }\end{array}$} & \multicolumn{4}{|c|}{$\begin{array}{c}\text { Hoe karakteriseert u de aandrang? } \\
\text { Om cirkel het getal dat van toepassing is: } \\
0=\text { Geen aandrang } \\
\text { 1= Normale aandrang: Ik kan } 30 \text { minuten uitstellen } \\
\text { 2= sterke aandrang: Ik kan max } 10 \text { mimuten uitstellen } \\
\text { 3=plotse / hevige aandrang: Ik moet NU gaan plassen! }\end{array}$} \\
\hline & & Tijdstip & Hocveelheid & & & & & & \\
\hline $9: 20$ & $400^{\mathrm{ml}}$ & $7: 00$ & $350 \mathrm{ml}$ & - & Leeg $\longmapsto+1 \quad x+\left.\right|^{\text {Vol }}$ & $\begin{array}{c}0 \\
\text { Geen }\end{array}$ & $\begin{array}{c}1 \\
30 \mathrm{~min}\end{array}$ & $\underset{10 \mathrm{~min}}{(2)}$ & $\begin{array}{c}3 \\
3 \\
\text { NU! }\end{array}$ \\
\hline $10: 00$ & $300^{\mathrm{ml}}$ & $10: 55$ & $510 \mathrm{ml}$ & - & Leeg $\longmapsto+\left.x\right|^{\text {Vol }}$ & $\begin{array}{c}0 \\
\text { Geen }\end{array}$ & $\begin{array}{c}1 \\
30 \min \end{array}$ & $\begin{array}{c}2 \\
10 \min \end{array}$ & (3) \\
\hline $13: 20$ & $250^{\mathrm{ml}}$ & $16: 00$ & $600^{\mathrm{ml}}$ & - & $\left.{ }^{\text {Leeg }} \longmapsto \quad \mathrm{X}\right|^{\text {Vol }}$ & $\begin{array}{c}0 \\
\text { Geen }\end{array}$ & $\begin{array}{c}1 \\
30 \min \end{array}$ & $\begin{array}{c}2 \\
10 \mathrm{~min}\end{array}$ & (3) \\
\hline $16: 15$ & $250^{\mathrm{ml}}$ & 19:20 & $260^{\mathrm{ml}}$ & - & $x+1^{\text {vol }}$ & $\begin{array}{c}0 \\
\text { Geen }\end{array}$ & (1) & $\begin{array}{c}2 \\
10 \min \end{array}$ & $\begin{array}{c}3 \\
\text { NU! }\end{array}$ \\
\hline F:20 & $150^{\mathrm{ml}}$ & $22: 00$ & $430^{\mathrm{ml}}$ & - & Leeg & $\begin{array}{c}0 \\
\text { Geen }\end{array}$ & (1) & $\begin{array}{c}2 \\
10 \min \end{array}$ & $\begin{array}{c}3 \\
\text { NU! }\end{array}$ \\
\hline $10: 00$ & $40^{\mathrm{ml}}$ & $-\div-$ & $\mathrm{ml}$ & & Leeg $\longmapsto 1$ & $\begin{array}{c}0 \\
\text { Geen }\end{array}$ & $\begin{array}{c}1 \\
30 \min \end{array}$ & $\begin{array}{c}2 \\
10 \min \end{array}$ & $\begin{array}{c}3 \\
\text { Nu! }\end{array}$ \\
\hline $20: 40$ & $300^{\mathrm{ml}}$ & $-\div$ & $\mathrm{ml}$ & & Leeg $\longmapsto 1$ & $\begin{array}{c}0 \\
\text { Geen }\end{array}$ & $\begin{array}{c}1 \\
30 \mathrm{~min}\end{array}$ & $\begin{array}{c}2 \\
10 \min \end{array}$ & $\begin{array}{c}3 \\
\text { NU! }\end{array}$ \\
\hline$-\div$ & $\mathrm{ml}$ & $-\div$ & $\mathrm{ml}$ & & Leeg $\longmapsto 1$ & $\begin{array}{c}0 \\
\text { Geen }\end{array}$ & $\begin{array}{c}1 \\
30 \mathrm{~min}\end{array}$ & $\begin{array}{c}2 \\
10 \min \end{array}$ & $\begin{array}{c}3 \\
\text { NU! }\end{array}$ \\
\hline$-\div$ & $\mathrm{ml}$ & $-\div-$ & $\mathrm{ml}$ & & Leeg $\left.\longmapsto 11\right|^{\text {Vol }}$ & $\begin{array}{c}0 \\
\text { Geen }\end{array}$ & $\begin{array}{c}1 \\
30 \min \end{array}$ & $\begin{array}{c}2 \\
10 \min \end{array}$ & $\begin{array}{c}3 \\
\text { Nu! }\end{array}$ \\
\hline$-\div$ & $\mathrm{ml}$ & $-:-$ & $\mathrm{ml}$ & & Leeg $\longmapsto 1,\left.\right|^{\text {Vol }}$ & $\begin{array}{c}0 \\
\text { Geen }\end{array}$ & $\begin{array}{c}1 \\
30 \mathrm{~min}\end{array}$ & $\begin{array}{c}2 \\
10 \mathrm{~min}\end{array}$ & $\begin{array}{c}3 \\
\text { NU! }\end{array}$ \\
\hline$-\div$ & $\mathrm{ml}$ & $-\because-$ & $\mathrm{ml}$ & & Leeg $\longmapsto 1$ & $\begin{array}{c}0 \\
\text { Geen }\end{array}$ & $\begin{array}{c}1 \\
30 \mathrm{~min}\end{array}$ & $\begin{array}{c}2 \\
10 \mathrm{~min}\end{array}$ & $\begin{array}{c}3 \\
\text { NU! }\end{array}$ \\
\hline$-{ }_{-}$ & $\mathrm{ml}$ & -:- & $\mathrm{ml}$ & & Leeg $\left.\longmapsto 11\right|^{\text {Vol }}$ & $\begin{array}{c}0 \\
\text { Geen }\end{array}$ & $\begin{array}{c}1 \\
30 \min \end{array}$ & $\begin{array}{c}2 \\
10 \mathrm{~min}\end{array}$ & $\begin{array}{c}3 \\
\text { NU! }\end{array}$ \\
\hline$-\div$ & $\mathrm{ml}$ & $-:-$ & $\mathrm{ml}$ & & Leeg $\longmapsto 1$ & $\begin{array}{c}0 \\
\text { Geen }\end{array}$ & $\begin{array}{c}1 \\
30 \min \end{array}$ & $\begin{array}{c}2 \\
10 \min \end{array}$ & $\begin{array}{c}3 \\
\text { NU! }\end{array}$ \\
\hline
\end{tabular}

\section{What do we need?}

In order to prevent miscommunication and to further unravel the aetiology of pathological conditions, such as $\mathrm{OAB}$, we need a clear and unambiguous terminology to describe normal and pathological bladder sensations based on descriptive terms that are understood and can be used by healthy subjects and patients. Furthermore, this terminology should mean the same to patients and physicians and ideally, it should be developed in every language separately. As mentioned before, sensations are subjective and the development of clear and unambiguous terminology is therefore a real challenge. Using questionnaires to develop such terminology is difficult because open ended questions will lead to many different answers and closed ended questions will force the subject to relate their own sensations to terminology used by the examiner. Discussions with subjects, so called focus group research, can be a better method to evaluate subjective information. In the field of psychology this method is already often used in order to evaluate ideas, opinions and assess bodily signals. By means of discussions between the subjects, participants can be stimulated to describe certain topics and give examples. Due to multiple sessions with the same subject and the presence of the researcher, ambiguities can be clarified directly. The interaction between all participants is another benefit of this method. Participants might come up with ideas or examples due to the comments of a different participant. During our pilot focus group sessions with healthy volunteers and patients with $\mathrm{OAB}$, the interaction was a positive experience for all subjects because they said that by the group discussion they were able to better formulate their own opinion. In our final method design we choose to combine focus group discus- 
sions with filling out different questionnaires. The discussions were merely used in order to clarify the terminology regarding bladder sensations, while the questionnaires were used to estimate the location of sensations and evaluate the pattern of the development of bladder sensations. The latter was done by asking a subject to fill out their sensation on an empty graph regularly, with time on the x-axis and intensity of the sensation on the $y$-axis. This method was chosen in order to avoid influencing the subject by examples of patterns and giving them the opportunity to estimate their sensation as being either increased or decreased during the same bladder filling.

Second of all, it is important that bladder sensation can be studied by means of a technique that is non-invasive and gives the opportunity to evaluate the entire pattern of bladder sensation ranging from no sensation to an absolute need to void. A controlled non-invasive bladder fast filling is, in our opinion, only achievable by means of a water loading protocol. This entails that subjects are given such a large intake so that the diuresis (production of urine) is maximum. During our water loading protocol the participants were asked to drink $1,000 \mathrm{ml}$ of water, in one hour before the start of the focus group session. During the session they were asked to drink another $200 \mathrm{ml}$ every $10 \mathrm{~min}$, with a maximum of an extra $1,000 \mathrm{ml}$. This water loading protocol was chosen to reach maximum bladder capacity by natural diuresis over a period of about 1 hour based on preliminary work.

Third of all, the relation between bladder sensation and voided volume had to be clarified. In order to do so we used the SR-BD. Not only did we use the urge scale, but we also introduced a visual analogue scale in order to be able to evaluate bladder fullness in a continuous manner. We have chosen this method based on neurophysiological studies in animals. During these studies, it has been shown that afferent activity is sent up the spinal cord to the brain stem, where bladder filling/volume is continuously measured $^{36}$. From that point of view, the ideal scale to grade bladder volume would be a continuous one.

Finally, we needed to compare bladder sensations experienced at home (uncontrolled setting) with bladder sensations experienced in the hospital (controlled setting) in order to evaluate possible effects of distraction, emotions, presence of other people etc. In order to do so, we compared our data on the SR-BD (filled out in an uncontrolled setting) with the data gathered during a controlled bladder filling sessions in the hospital (controlled setting).

\section{Aim}

In this thesis we focus on (1) describing, developing and refining new techniques to evaluate bladder sensations and (2) studying the differences in bladder sensations between patients with $\mathrm{OAB}$ and healthy volunteers. The following questions were the basis of the studies described in this thesis:

- $\quad$ Can bladder sensations be studied during a controlled non-invasive bladder filling by means of a water loading protocol?

- Which words do healthy volunteers or patients with $O A B$ use to describe bladder sensations?

- How does the absolute need to void develop in healthy volunteers and patients with the overactive bladder syndrome (continuous or episodic)? 
- Can a grading of the perception of bladder fullness or the degree of urge replace the measurement of voided volumes on a sensation-related bladder diary?

- $\quad$ Are bladder sensations experienced at home different from bladder sensation experienced at the hospital?

\section{Outline}

Chapter 1 gives an introduction on bladder sensations and techniques to study these sensations. Chapter 2 reviews our current knowledge on bladder sensations and argues on the necessity of a clear terminology and proposes a re-examination of bladder sensation, sensation development and their use as a diagnostic technique. Furthermore, it proposes an alternative model describing how peripheral information generating "introspective sensations" and "voiding sensations" might be different but interrelated.

The reliability of the degree of urge scale and perception of bladder fullness scale and the relationship between both scales and bladder volume are discussed in chapter 3 . This chapter also describes possible differences between bladder sensation experienced in a controlled and an uncontrolled setting.

In order to standardise and validate new techniques to evaluate bladder sensation we describe a new non-invasive method to study the development of physiological bladder sensations in chapter 4 and evaluate the repeatability of this method.

To introduce a clear and unambiguous terminology for bladder sensations we studied the normal bladder sensations in healthy volunteers by means of focus group research, this is described in chapter 5 . The differences between normal and pathological bladder sensations during a non-invasive bladder filling are discussed in chapter 6 .

To summarise, this thesis describes new methods to evaluate normal and pathological bladder sensations and describes the results that were found when using these techniques in healthy volunteers and patients with the overactive bladder syndrome.

\section{References}

1. Denny-Brown, D., Robertson, E. G.: On the physiology of micturition. Brain, 56: 149, 1933

2. Nathan, P. W.: Sensations associated with micturition. Br J Urol, 28: 126, 1956

3. Wyndaele, J. J.: The normal pattern of perception of bladder filling during cystometry studied in 38 young healthy volunteers. J Urol, 160: 479, 1998

4. Wyndaele, J. J., De Wachter, S.: Cystometrical sensory data from a normal population: comparison of two groups of young healthy volunteers examined with 5 years interval. Eur Urol, 42: 34, 2002

5. Abrams, P., Cardozo, L., Fall, M. et al.: The standardisation of terminology of lower urinary tract function: report from the Standardisation Sub-committee of the International Continence Society. Am J Obstet Gynecol, 187: 116, 2002

6. Chapple, C. R., Artibani, W., Cardozo, L. D. et al.: The role of urinary urgency and its measurement in the overactive bladder symptom syndrome: current concepts and future prospects. BJU Int, 95: 335, 2005

7. Gillespie, J. I., G. A. van Koeveringe, et al.: On the origins of the sensory output from the bladder: the concept of afferent noise. BJU Int 103: 10, 2009.

8. Stewart, W. F., Van Rooyen, J. B., Cundiff, G. W. et al.: Prevalence and burden of overactive bladder in the United States. World J Urol, 20: 327, 2003

9. Irwin, D. E., Milsom, I., Hunskaar, S. et al.: Population-based survey of urinary incontinence, overactive bladder, and other lower urinary tract symptoms in five countries: results of the EPIC study. Eur Urol, 50: 1306, 2006

10. Abrams, P., Kelleher, C. J., Kerr, L. A. et al.: Overactive bladder significantly affects quality of life. Am J Manag Care, 6: S580, 2000 


\section{Chapter 1}

11. Burgio, K. L., Kraus, S. R., Menefee, S. et al.: Behavioral therapy to enable women with urge incontinence to discontinue drug treatment: a randomized trial. Ann Intern Med, 149: 161, 2008

12. Morrison, J. F. B., Steers, W. D., Brading, A. et al.: Neurophysiology and neuropharmacology. In: Incontinence, 2nd International Consultation on Incontinence. Edited by P. Abrams, L. Cardozo, S. Khoury et al. Plymouth: Health Publication Ltd, pp. 83-163, 2002

13. Hyman, M. J., Groutz, A., Blaivas, J. G.: Detrusor instability in men: correlation of lower urinary tract symptoms with urodynamic findings. J Urol, 166: 550, 2001

14. Oliver, S., Fowler, C., Mundy, A. et al.: Measuring the sensations of urge and bladder filling during cystometry in urge incontinence and the effects of neuromodulation. Neurourol Urodyn, 22: 7, 2003

15. Robertson, A. S., Griffiths, C. J., Ramsden, P. D. et al.: Bladder function in healthy volunteers: ambulatory monitoring and conventional urodynamic studies. Br J Urol, 73: 242, 1994

16. Kulseng-Hanssen, S., Kristoffersen, M.: Urethral pressure variations in females with and without neurourological symptoms. Scand J Urol Nephrol Suppl, 114, 1988.

17. Kulseng-Hanssen, S.: Prevalence and pattern of unstable urethral pressure in one hundred seventy-four gynecologic patients reffered for urodynamic investigation. Am J Obstet Gynecol, 146, 1983.

18. FitzGerald, M.P., Kenton, K.S., Brubaker, L.: Localization of the urge to void in patients with painful bladder syndrome. Neurourol Urodyn, 24: 7, 2005.

19. Blaivas, J. G., Panagopoulos, G., Weiss, J. P. et al.: Two types of urgency. Neurourol Urodyn, 28: 188, 2009

20. Blaivas, J. G.: Overactive bladder and the definition of urgency. Neurourol Urodyn, 26: 757, 2007

21. De Wachter, S., Hanno, P.: Urgency: all or none phenomenon? Neurourol Urodyn, 29: 616, 2010

22. Digesu, G. A., Khullar, V., Panayi, D. et al.: Should we explain lower urinary tract symptoms to patients? Neurourol Urodyn, 27: 368, 2008

23. Erdem, E., Tunckiran, A., Acar, D. et al.: Is catheter cause of subjectivity in sensations perceived during filling cystometry? Urology, 66: 1000, 2005

24. De Wachter, S., De Laet, K., Wyndaele, J. J.: Does the cystometric filling rate affect the afferent bladder response pattern? A study on single fibre pelvic nerve afferents in the rat urinary bladder. Neurourol Urodyn, 25: 162, 2006

25. Erdem, E., Akbay, E., Doruk, E. et al.: How reliable are bladder perceptions during cystometry? Neurourol Urodyn, 23: 306, 2004

26. De Wachter, S., Van Meel, T. D., Wyndaele, J. J.: Can a faked cystometry deceive patients in their perception of filling sensations? A study on the reliability of spontaneously reported cystometric filling sensations in patients with non-neurogenic lower urinary tract dysfunction. Neurourol Urodyn, 27: 395, 2008

27. Dompeyre, P., Fritel, X., Bader, G. et al.: Bladder sensitivity testing using a visual analogue scale: comparative cystometric study on women. Neurourol Urodyn, 26: 350, 2007

28. Abrams, P., Klevmark, B.: Frequency volume charts: an indispensable part of lower urinary tract assessment. Scand J Urol Nephrol Suppl, 179: 47, 1996

29. De Wachter, S., Wyndaele, J. J.: Frequency-volume charts: a tool to evaluate bladder sensation. Neurourol Urodyn, 22: 638, 2003

30. Naoemova, I., De Wachter, S., Wyndaele, J. J.: Comparison of sensation-related voiding patterns between continent and incontinent women: a study with a 3-day sensation-related bladder diary (SR-BD). Neurourol Urodyn, 27: 511, 2008

31. Naoemova, I., Van Meel, T., De Wachter, S. et al.: Does sensory bladder function during cystometry differ from that in daily life? A study in incontinent women. Neurourol Urodyn, 28: 309, 2009

32. Honjo, H., Kawauchi, A., Ukimura, O. et al.: Analysis of bladder diary with urinary perception to assess overactive bladder symptoms in community-dwelling women. Neurourol Urodyn, 2009

33. Digesu, G. A., Basra, R., Khullar, V. et al.: Bladder sensations during filling cystometry are different according to urodynamic diagnosis. Neurourol Urodyn, 28: 191, 2009

34. Cartwright, R., Panayi, D., Cardozo, L. et al.: Reliability and normal ranges for the Patient's Perception of Intensity of Urgency Scale in asymptomatic women. BJU Int, 105: 832, 2009

35. Coyne, K. S., Margolis, M. K., Hsieh, R. et al.: Validation of the urinary sensation scale (USS). Neurourol Urodyn, 30: 360, 2011

36. Shea, V. K., Cai, R. et al.: Sensory fibers of the pelvic nerve innervating the Rat's urinary bladder. J Neurophysiol, 84: 4, 2000 


\section{On the nature of bladder sensation: the concept of sensory modulation}

S. G. G. de Wachter ${ }^{1,2}$, R. Heeringa ${ }^{1,2}$, G. A. van Koeveringe ${ }^{1,2}$, J. I. Gillespie $^{3}$

1. Department of Urology

Maastricht University Medical Centre, Maastricht, The Netherlands

2. European Graduate School of Neuroscience (EURON)

Maastricht University Medical Centre; The Netherlands

3. Uro-physiology Research Group

Medical and Dental School, University of Newcastle Upon Tyne, England

Neurourology and Urodynamics - 30 (7): 1220-6, 2011

Epub: 2011 June 9 


\begin{abstract}
Going to the toilet is an essential every day event. Normally, we do not give much thought to the sensations and factors that trigger voiding behaviour: we just go. For many people, this apparently simple task is complicated and dominates their life. They have strong sensations and sudden desires to void, often resulting in incontinence. It is therefore important that we understand the origins for this functional change and identify means to alleviate it.

A considerable body of work has focused on this problem and ideas and concepts on the nature of bladder sensations are embedded in the literature. However, careful reviewing of this work and the established ideas suggests that we might be missing something and the problem may be much more complex.

In this paper we argue the necessity to return to first principles and a re-examination of the problem. We explore the use of focus groups to identify relevant bladder sensation and what triggers 'bladder' behaviour. We argue that there are differences in what can be described as 'introspective bladder sensations' and the sensations reported immediately before a void, 'void sensations'. Finally, we propose an alternative model describing how peripheral information generating 'introspective sensations' and 'void sensations' might be different but interrelated sensations. By exploring such ideas and identifying such complexity it is our intention to stimulate debate and generate further research in the field in order to understand better the physiology of bladder sensation and the pathology of increased urge, frequency and incontinence.
\end{abstract}




\section{Introduction}

Healthy people normally do not dwell upon their bladder. The lower urinary tract is designed to store urine to allow daily activities without continuous intrusions of introspection and checking on the necessity to void. Our awareness of our bladder appears to come and go in our daily consciousness. In order to achieve this apparently simple sequence of events a whole range of complex interacting systems are involved: the generation of information from the lower urinary tract, processing in the spinal cord, pontine centres, thalamus, hypothalamus and cortex (Figure 1) ${ }^{1}$. The control of our bladder and of our voiding behaviour is, in reality, a complex interplay of sensory and cognitive processes linked to coordinating reflexes.

This system can go wrong and lower urinary tract disorders are common. These have been linked together and described as 'The Overactive Bladder Syndrome' $(O A B)^{2}$. The major symptoms of $\mathrm{OAB}$ are urgency, increased frequency of voiding often with incontinence. It is now becoming clear that the key changes in $O A B$ are related to altered sensation. If we are to understand the origins and nature of $O A B$ and identify and treat the condition it is essential that we understand fully the origins of sensation and sensory processing.

A great deal of work has been done to identify the systems generating afferent information from the bladder: 'afferent noise' ${ }^{, 3}$. Although complex, the properties and potential roles of the different components are emerging ${ }^{4,5}$. However, clear and objective data on the conversion of the key components of 'afferent noise' to sensation, and the way sensation and perception have impact on behaviour are still poorly understood.

The purpose of this paper is to concentrate on sensation and to review critically the relevant literature. The 'established' views, terminology and methodology can be traced to observations made over 70 years ago. Some of the terminology and the ideas from this early work is still valid but it has been gradually altered and represented differently to such an extent that the current dogma must be seriously questioned. By challenging the accepted ideas, terminology and concepts we are attempting to stimulate others to focus on this basic problem of everyday life.

\section{Current views on bladder sensation-The International Continence Society Terminology}

Current views on bladder sensation are derived largely from urodynamic studies. In 1933, Denny-Brown and Robertson described a specific pattern of bladder sensations during artificial bladder filling ${ }^{6}$. They described sensations of pressure, desire, fullness and discomfort or pain, but disagreed on the character of the sensation: episodic or continuous. Later Nathan also pointed out that different sensations might be originating from different anatomical locations ${ }^{7}$. These ideas and terms remained in use until the 1990 's. Then, in a series of cystometric studies using healthy subjects, volunteers were asked to report their sensations as the bladder was filled ${ }^{8,9}$. In these studies, no explanation was given to the volunteers as to what they might perceive. Yet all volunteers mentioned spontaneously the same pattern consisting of a first sensation of filling, first desire to void and strong desire to void. It is not clear from this work whether this termi- 
nology came from the subjects themselves, or was predetermined by the observer. The terminology used included ${ }^{8,9}$ :

- $\quad$ First sensation This was vague, it waxed and waned and was localized in the lower pelvis. It could easily be ignored for 30 seconds to 2 minutes.

- $\quad$ First desire A first desire to void was recognized as a familiar constant sensation that persuades a person to seek a place to void, or void at the next convenient moment, but voiding could be delayed. It was reported in the lower abdomen and did not disappear during further filling but became gradually stronger. The volunteers could ignore this sensation with increasing difficulty and never for longer than 20 seconds.

- $\quad$ Strong desire A strong desire to void corresponded with a constant almost uncomfortable sensation in the perineal region or urethra and was further described as a strong urge or great tension. It was also described as a persistent desire to void without the fear of leakage. At that moment, the bladder was considered full and the filling was asked to stop.

Based on these studies, the pattern of sensations and terminology were considered and accepted as the norm. They were given strength and credence by the endorsement of the Standardization Committee of the International Continence Society ${ }^{2}$. It was thus 'established' that there were different sensations during filling, that were easily distinguishable from each other and that they were episodic in nature (see Figure 2).

Figure 1 shows the complexity of the interacting systems as deducted from animal tracing studies (A), and human brain imaging studies (B). A close interaction between areas dealing with unconscious processing and integration of afferent noise and areas involved in the conscious processing and perception of this afferent noise leads to the sensation of bladder filling which we use to incorporate voiding into daily life activities. Reproduced from ref. 1.

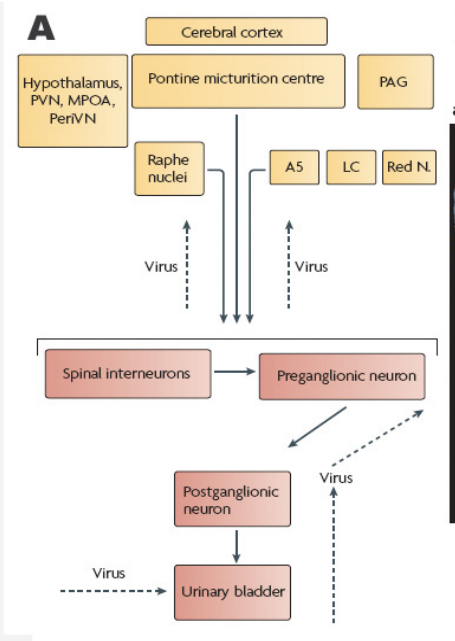

$\mathbf{B}$
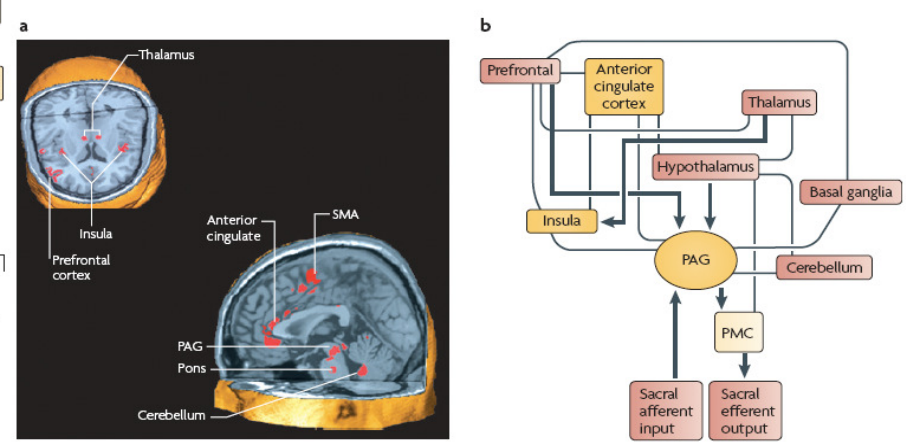
Figure 2 shows A. The normal sensations of bladder filling are considered to be episodic, but the duration, intensity and frequency show an increase in relation to increasing volume. The cycle terminates with a void that may or may not be associated with a strong desire. This does not take into account the findings that different bladder volumes can occur with different sensations and for a given intensity of sensation, a large range of volumes can be voided (cfr infra). B. Based on the concept in A, it is suggested that in patients with OABS the normal episodic sensations may occur, but the sequence can be interrupted by the occurrence of urgency, which is considered a different pathological sensation, which persists until a void has taken place or incontinence occurred. Reproduced from Chapple et al. ${ }^{10}$

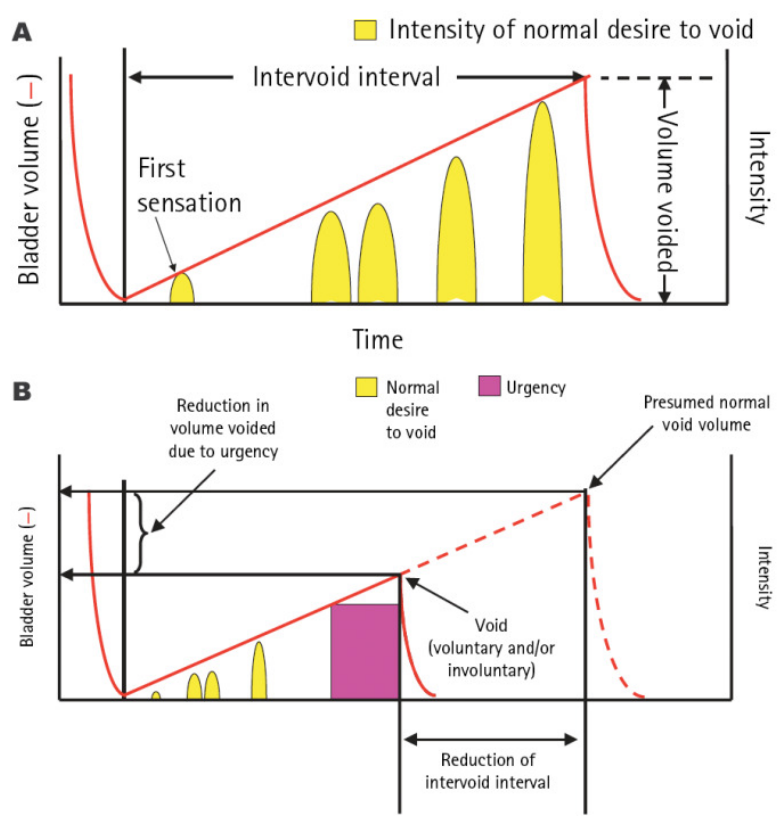

\section{Critical reflections on cystometric bladder sensation}

Before accepting the normal pattern of sensation and terminology it is essential to be certain that the study designs are appropriate to conclude that there are different filling sensations, that the terminology is precise and robust, that filling sensations are episodic and that the sensations originate from different locations.

\section{Terminology of bladder sensation}

In studies examining the "normal pattern of filling sensations", volunteers were often asked to describe and localize all sensations. They were then asked to ignore these sensations and indicate when they returned ${ }^{8}$. Just giving these instructions may bias the results. Asking subjects to localize "all" sensations implies that there are going to be different sensations. Furthermore, asking subjects to ignore a sensation and report its return makes the sensation episodic by default not necessarily in reality. If the sensation "returns", is it the same sensation or is it different? Does it recur in the same place or has it moved to a slightly different location lower in the abdomen or in the perineum? Would the volunteers have given the same descriptions if they were asked to focus on the first sensation instead of trying to ignore it, and to describe the change in sensation or in intensity? On the other hand, these studies do not address whether the sensations were 
different in character, were they perceived as distinct sensations or did they represent an increase in intensity of the same sensation. We do not know, because these key alternative questions were never addressed.

In other studies on bladder sensation, an explanation was given before the study to describe what the volunteers and patients could expect to perceive during a filling cystometry $^{9,11,12}$. Perhaps not surprisingly, when questioned they all reported the specific pattern of sensations, as proposed by the observers. Would the subjects have reported the same sensations if they had not been instructed on what to perceive? We do not know. Yet the consequences of telling subjects what to perceive possibly invalidates the aim of the observation, namely to assess sensation.

Erdem and co-workers did an interesting experiment on this topic. Clear instructions were given orally and in writing to patients during cystometry regarding the sensations they would experience ${ }^{11,12}$. Importantly, and cunningly, no fluid was infused into the bladder. The subjects were asked what they felt as time passed and many reported changing sensations that followed the expected pattern: $83 \%$ perceived first sensation of filling, $80 \%$ first desire to void and even $25 \%$ strong desire to void. The same protocol was repeated by another group and showed completely opposite results: out of 59 patients, only three spontaneously reported a first sensation of filling, one a first desire to void and none a strong desire to void ${ }^{13}$. This illustrates dramatically the major impact an experimenter has on the outcome of an experiment. We must therefore be cautious and question study designs where information, uncontrolled questioning and cystometry are used in combination.

\section{Sensations and Visual Analogue Scales (VAS)}

If language is a problem exploring bladder sensation this can be circumvented using non-verbal techniques, for example by visual analogue scales. Dompeyre and coworkers studied bladder sensation in patients during two consecutive cystometric fillings. During one fill patients were asked to report all the sensations they experienced. In the second fill, patients used a continuous VAS scale to reflect sensation ${ }^{14}$. They showed that all patients could describe a specific pattern of different sensations. When in the same individuals bladder sensation was evaluated using a VAS scale a period of no sensation was followed by one sensation of a desire to void that continuously increased in intensity until the bladder was completely full. This raises the question 'what actually is perceived when the bladder is filled' and also 'whether patients actually report what they feel, or just what they think they should feel'? This example clearly shows that the information that is given to the subject under evaluation will largely determine the resulting data and again stresses the importance to obtain "true data" from the subjects eliminating the observer's influence in order to obtain unbiased data.

\section{Anatomical evidence for different sensations}

The idea that there are different filling sensations can be traced to studies over 50 years ago that have been "reported" to provide evidence to show that different filling sensations are conducted through different peripheral neural pathways ${ }^{7,15}$. The studies of Riddoch ${ }^{16}$ and Gunterberg ${ }^{17}$ are cited as evidence that the first sensation of filling is carried in the hypogastric nerve $e^{8,9,18}$. 
However, careful reading of the original Riddoch manuscript suggests that this is not true. The subjects described had bilateral complete lesions of the cauda equina involving the last four sacral posterior roots, thus leaving only the inferior hypogastric splanchnic nerves intact. Riddoch actually reported that each individual experienced 'mild discomfort', an awareness that the bladder was full and a desire to micturate. If urine was removed this "mild discomfort" disappeared, but if not, the sensation increased in intensity $^{16}$. Importantly, this description demonstrates that if the sacral roots are destroyed, the normal gradual building up of bladder sensation is absent. There is no link to 'first sensation'. The observations do however suggest a key role for the sacral roots in carrying information on bladder volume.

Gunterberg described patients with lesions of the sacral nerves. Stretches of the detrusor wall with a ureteral catheter could be felt on the denervated side, indicating that this sensation was not mediated in sacral nerves, but probably in the hypogastric nerves ${ }^{17}$. Stretching the bladder wall with a ureteral catheter is very localised stretch and does not resemble a more homogenous stretch induced by bladder filling. It is thus unclear how evidence for activity of the hypogastric nerve can be correlated to the first sensation of filling.

The views on different nerves carrying sensations are also very difficult to reconcile with the animal data from afferent recordings that show activity from early in the filling phase in both the hypogastric and pelvic nerve ${ }^{5,19-22}$. So, after revisiting these often-cited studies, there is no real evidence to support the view that different filling sensations are conducted through different peripheral nerves. Data on the central pathways that convey sensory information is less clear and information on the central processing is currently emerging from imaging studies, which still needs to be correlated to different bladder sensations. An anatomical basis for perceiving different sensations is therefore wide open.

\section{The development of voiding diaries}

Cystometry allowed the experimenter to control accurately bladder volume. However, a major criticism is that it is invasive and uses artificially fast bladder filling rates. Presence of a urethral catheter in itself induces sensation in both urethra and bladder ${ }^{12}$. How a subject might differentiate between such instrumental sensation and real sensations related to volume is impossible to evaluate. Also the high filling rates used during cystometry will almost certainly influence afferent nerve firing ${ }^{23}$ and perception of a full blad$\mathrm{der}^{24}$.

To overcome partially these difficulties, the "sensation related bladder diary" was introduced $^{25}$ asking subjects to note, at every void, their perception of bladder fullness according to a predefined scale (table 1 ) and the voided volume.

It was found that the mean volumes voided, at every grade of fullness, were significantly different and that there was a correlation between voided volume and grade of bladder fullness $^{25-27}$ (see figure 3). However, it is obvious that each sensation is reported over an enormous volume range ${ }^{25,26,28}$.

The bladder diary is an essential tool for proper clinical evaluation, and in addition might be a good tool to study bladder sensation non-invasively. It must be remembered that it still has the complication of language and the difficulties a subject has in mapping exper- 
imenter generated terms to his or her own sensation. Furthermore, diaries do not actually address the question how sensation develops during filling, but only provide information that relates to sensations immediately before an individual goes to the toilet.

We should not take it for granted that our "familiar" terminology has the same meaning for the experimenter and the subjects. For example, it has been shown that even basic clinically used terms such as 'frequency' and 'urgency' have a different meaning for observers and $30-40 \%$ of the patients, and that $20 \%$ of the patients doesn't know the meaning of these terms ${ }^{29}$.

This problem in terminology becomes even more complicated if we consider that different patients groups may have a different perception of bladder sensation during filling according to their bladder dysfunction ${ }^{30}$. Considering this, it is not surprising that physicians still struggle with questions such as "is urgency an increased normal sensation or a different pathological sensation? ${ }^{31}$ “ or "Is urgency an all or none phenomenon" ${ }^{32}$ ?

Table 1 shows the definition of each score on the degree of urge scale

\begin{tabular}{|l|l|}
\hline 0 & No bladder sensation \\
\hline 1 & $\begin{array}{l}\text { First sensation of filling; Voiding can easily be delayed for more than } 60 \\
\text { minutes No desire to void }\end{array}$ \\
\hline 2 & First desire to void; Voiding can be delayed for at least 30 minutes \\
\hline 3 & Strong desire to void; Voiding cannot be delayed for more than 15 minutes \\
\hline 4 & Urgent desire to void; Voiding cannot be delayed for more than 5 minutes \\
\hline
\end{tabular}

Figure 3 shows data from a 3 days voiding diary in 15 healthy female volunteers. Grade of perception was according to the definitions in table 1. Although there is a significant difference in voided volumes between consecutive grades, notice the large volume ranges and overlap at the different grades of perception. This suggests that factors other than grade of perception determine the voided volume.

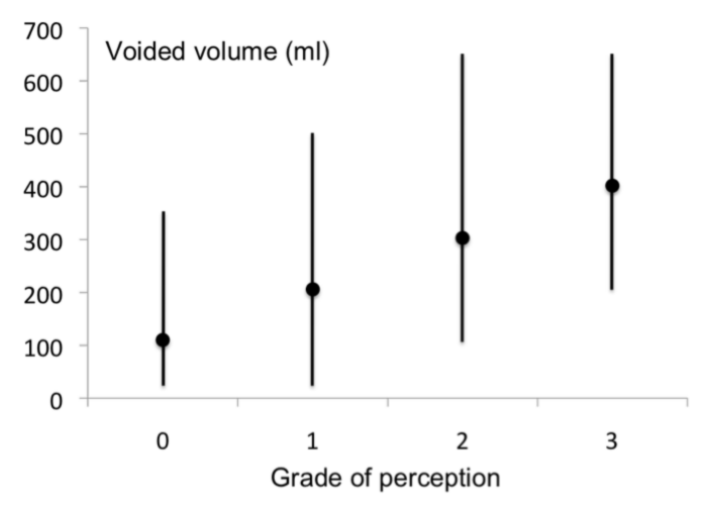




\section{Where to go now?}

The obvious place to start is right back at the beginning: simply ask normal subjects to describe what they feel as their bladder fills. This sounds easy, but it is not. The problem is communication. It is not obvious to a naïve subject what specific sensation he or she is being asked to focus on, particularly early in the filling phase. During the early phase there is almost certainly a sensation but it is weak, vague, not specific or localised. Subjects do not easily relate such sensations to the bladder. It is only when the bladder further fills that the sensations 'appear' and are easier to relate to 'the bladder' and 'need to go'. It is for this reason that we have perhaps become over-focused on the stronger sensations of urge and imminent voiding that drive people to the toilet. Therefore, we may be neglecting a great deal of perceptual information about the bladder because subjects neither recognise it nor know how to describe it. Also, the naïve subject has no detailed anatomical knowledge making it very difficult to describe or interpret the origin of lower abdominal sensation. Even physicians that investigate bladder sensation state that this sensation "is unusually difficult to describe" .

If we are to develop our understanding about the bladder sensation we have to address several key issue:

- what is the normal pattern of sensation during normal filling?

- where is it felt?

- $\quad$ can this easily be described bearing in mind that it relates to a process that takes several hours to complete?

- what agreed language, words and terminology should be used?

\section{The focus group approach}

One approach is to bring groups of naïve subjects, volunteers and patients, together (focus groups), 'establish' a 'controlled non-invasive' bladder filling and start discussions on bladder sensation. However, this in itself is not simple. If you inform naïve subjects about what you want from them you may bias the result, as discussed above. Alternatively, if you do not give guidance you can get such disparate data that is unintelligible and un-interpretable. This is a well-recognised problem in extracting opinion.

In order to get a 'natural' bladder filling, participants can be asked to increase their fluid intake prior to a session by simply drinking water. The increased diuresis can then be used to facilitate bladder filling and allow subjects to focus on sensation over a time scale of about 1 hour. To avoid observer bias and terminology bias, subjects are given a blank piece of paper with $x$ and $y$ axes drawn ( $x$ being explained to be time, $y$ intensity of bladder sensation). To enable an empty bladder at the start of the observation, subjects should void and then mark on the graph at 10-minute intervals the intensity of bladder sensation with $100 \%$ having a absolute need to void. At this sensation they void again and the voided volume is measured. Assuming a constant diuresis, from time elapsed and the volume voided it is possible to relate time to bladder volume. Figure 4 gives a typical example of such an approach (De Wachter et al. submitted). This pattern appears to be reproducible and resembles the cystometric observations on the continuous sensations reported using VAS scales ${ }^{14}$. 
During subsequent sessions, these volunteers can be asked, without prompting, to describe in their own words the sensations they feel as the bladder is filling. This generates a large number of descriptions involving words like 'pressure', 'ache', 'nagging', 'tingling'. Surprisingly, no two subjects ever used exactly the same terms. Towards the end of a fill more common words were used like 'pressure', 'need', 'desire ${ }^{, 33}$. Careful sifting of the words used by all of the individuals and discussion between the individuals can then allow consensus terms to emerge for different sensations as the bladder filled (Figure 5). The aim of the focus group approach is to repeat these discussion groups with new subjects until the obtained words and information does not change anymore. From this approach it seems possible to generate curves demonstrating how sensation changes and acquire words and terms to describe this progressive increase in sensation.

It would appear that bladder sensation, derived during an increased diuresis and with 'focused introspection', is continuous. It has recently been argued, after examining all the patterns of afferent discharge from the bladder, that information is constantly being streamed to the central nervous system ${ }^{3}$. Thus, it is not surprising that 'focused introspection' reveals a similar constant and progressive rise in sensation. However, this description of constant sensation is different from the pattern of sensations described and accepted by many who test patients using cystometry ${ }^{2}$. Here the sensations are described as episodic using terms like first awareness, first sensation to void, second sensation, urge (see figure 2). The question is now, 'can these apparently different accounts be reconciled'?

Figure 4 shows the introspective sensation curves drawn by a single subject on three occasions each separated by 10 days (adapted from De Wachter et al. submitted). The curves are very similar demonstrating a progressive increase in sensation as the bladder filled. Sensation was reported early in the fill and this increased, slowly at first, but continuously and progressively to a maximum which triggered a void. This graphs demonstrates the good repeatability determined in the same subject.

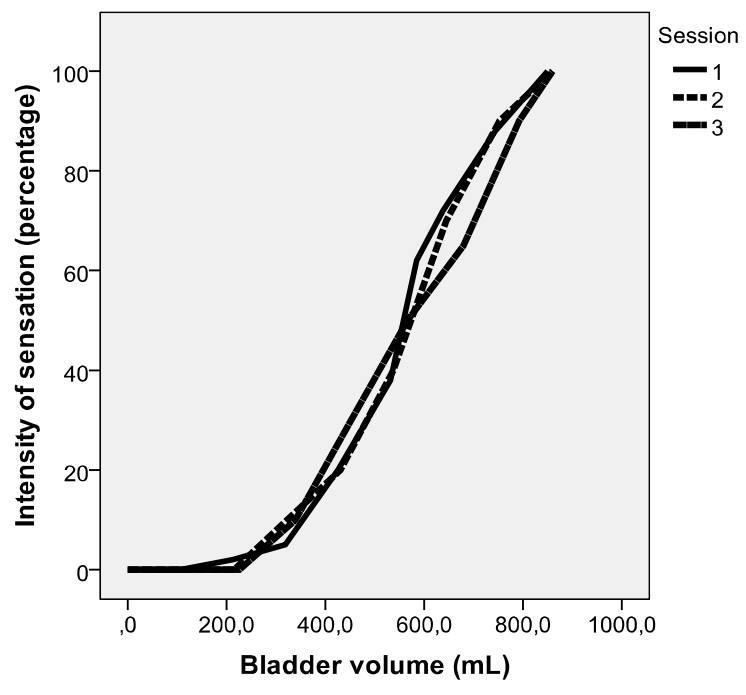


Figure 5 This diagram suggests a modified view of the perceived sensations as the bladder fills. The sensations generated during filling are considered to be a continuum not episodic. However, within the continuum different linked phases can be recognized each associated with a progressive intensification of sensation. The differences between this model and that in Figure 2 may not, at first glance, be too apparent. However, they represent basic differences in the fundamental physiological processes that contribute to bladder sensation. If the overall aim is to use such models to interpret clinical observations and assess the effects of drugs it is essential that the underlying concepts are based on our most up to date appreciation of the physiological, pharmacological and psychological processes underlying bladder sensation. Reproduced from ref. 3.

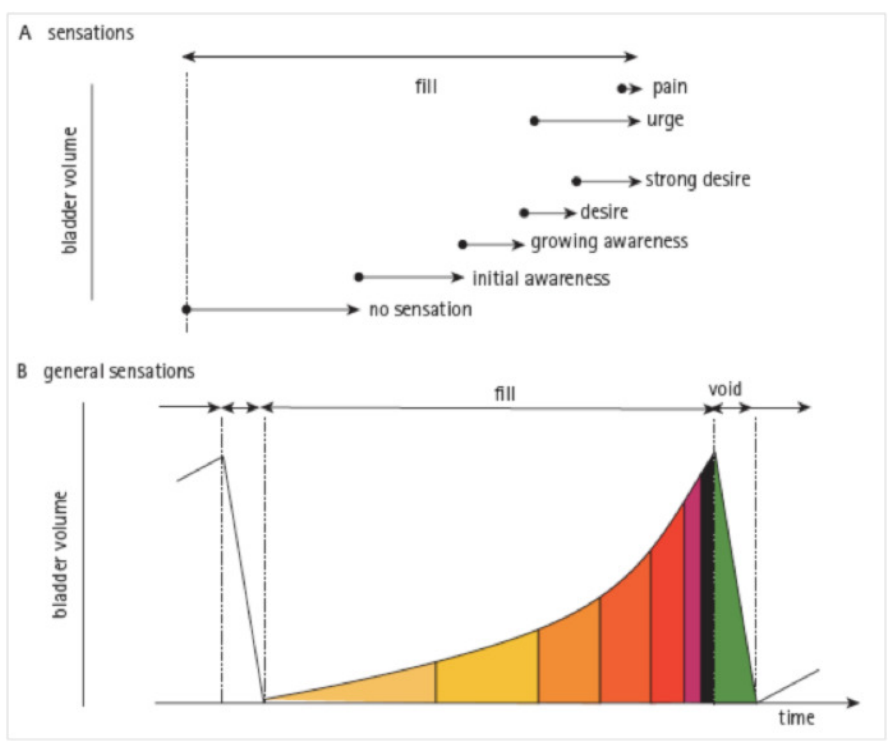

\section{Comparison of 'focused introspection' and voiding diary data}

An insight into this may be gleaned by comparing, in the same subjects 'focused introspection' and sensation reported on voiding diaries that use the same intensity scale as during the focused introspection. Figure 6 shows such data from one typical subject (Heeringa et al. in preparation). Based on the voiding diaries, voids are initiated at almost any sensation and the sensations reported are extremely variable: a well recognised phenomenon ${ }^{25-27,34}$. Interestingly, the 'void sensation' curve and the 'focused introspection' curves differ: the 'focused introspection' curve always lying below the 'void sensation' curve at all bladder volumes.

Clearly, the voiding diary data and focused introspection data are different. This may be potentially very important and very revealing. We have argued that the sensations generated from 'focused introspection' map well to the patters of afferent information being generated from the bladder ${ }^{3}$. Thus, this curve may represent a basal level of sensation emanating from the bladder. The fact, that at almost all volumes a greater sensation is reported at the time when a decision to void is made must mean that the reported prevoid sensations have been amplified and made 'more significant' to the observer. We hypothesize that there may have been a central modulation of sensory information, which represents the concept of 'sensory modulation'. It is possible that the sensitivity of the systems generating the afferent signals is increased. Indeed, there is some evidence that this might occur via regulation of motor/sensory noise ${ }^{3,35}$. Alternatively, the pro- 
cessing of the afferent information changes to accentuate the perceived sensations. This possibility is also tenable as considerable information is available on specific nuclei and their interconnections that receive and send inputs to the centres involved in controlling urine storage and voiding (Figure 7).

This psychological heightening of sensation in the immediate pre-void might be important as key behavioural issues have to be considered by the individual at this time (toilet seeking, social interactions, convenience, appropriateness). The implications of this are profound. It suggests that sensations reported from voiding diaries at the time of void are amplified and related to a very specific aspect of sensation in relation to bladder control.

Such an analysis suggests that the perception of bladder fullness has at least two components: one derived from the afferent information emanating from the bladder and a second modified, more intense, sensation generated as a result of central processing in order to direct behaviour immediately before a void. Therefore, when considering patients with OABS we should be aware that there might be both peripheral and central aspects. If the central amplification is exaggerated then subjects may amplify and interpret weak peripheral input incorrectly, experience exaggerated sensation and trigger 'unnecessary' voiding behaviour.

What is also clear from such approaches is that the sensations that normally trigger voiding behaviour are not those of urge or urgency. They are the milder sensations of generalised bladder awareness and fullness that trigger the complex behaviour leading up to and initiating voiding. This is not actually a new idea and it does appear, but hidden, in the literature ${ }^{25-28,34}$. The idea is infrequently discussed in the current work and discussions on bladder sensation and overactive bladder. If it is true that normal subjects trigger voiding behaviour using non-urge bladder sensations then what about those patients who present with urge and frequency? What do these patients really feel? Are the apparent increased sensations derived from the periphery or are they excessively amplified in the CNS? Understanding these questions is now one of the major tasks in functional urology. 
Figure 6 shows the typical example of relation between the "focused introspection curve" (dashed line) and the "void sensation curves" determined on the voiding diary data of one participant. The horizontal lines show the means and ranges of the voided volumes at a given sensation of fullness, as measured by the individual during three days. Note the wide overlap in voided volumes at the different intensities of fullness.

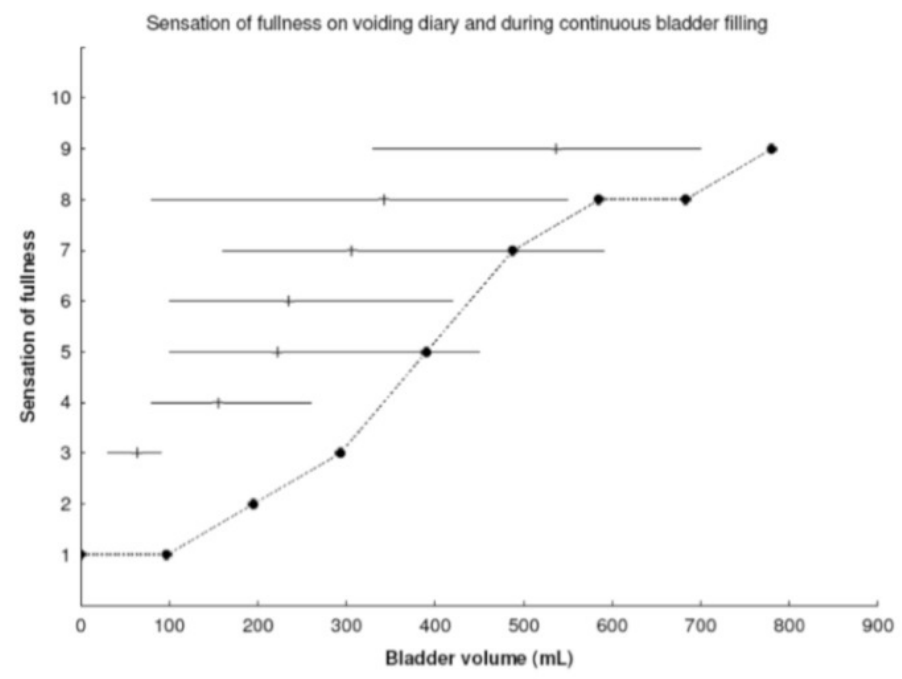

Figure 7 is a schematic diagram describing a possible scheme to account for different bladder sensations during filling and the sensations described as part of voiding behaviour. The most important aspect of this scheme is that an individual can effectively report two categories of bladder sensation: 'awareness' that can be accessed by introspection and 'pre-void' sensations that are related to the complex behaviour of voiding and activation of voiding reflexes. Central to this scheme is a 'comparator'. This is a hypothetical construct lying within the central nervous system. It is the point in the scheme where information from different sources are weighed and decisions are made. In the 'comparator', afferent information from the bladder it assessed and information related to bladder volume made available to consciousness. Here also, input from regions controlling complex behaviour are collected and used to determine if voiding is required either for social or practical purposes. The decision to void, ultimately made in the 'comparator', is therefore based on physical data (afferent noise) and cognitive processes (learned behaviour, convenience). As the bladder fills the awareness component grows and this input gains greater weight. Ultimately it dominates the system and a void is essential and unavoidable, if not incontinence ensues. Once the 'comparator' has integrated the awareness and behavioural elements a decision is made to void. As part of the next step in the process sensations associated with the bladder are amplified. This is the concept of 'sensory modulation'. This amplification focuses and drives voiding behaviour (toilet seeking, social behaviour, physical readiness) and prepares the voiding reflexes to be activated.

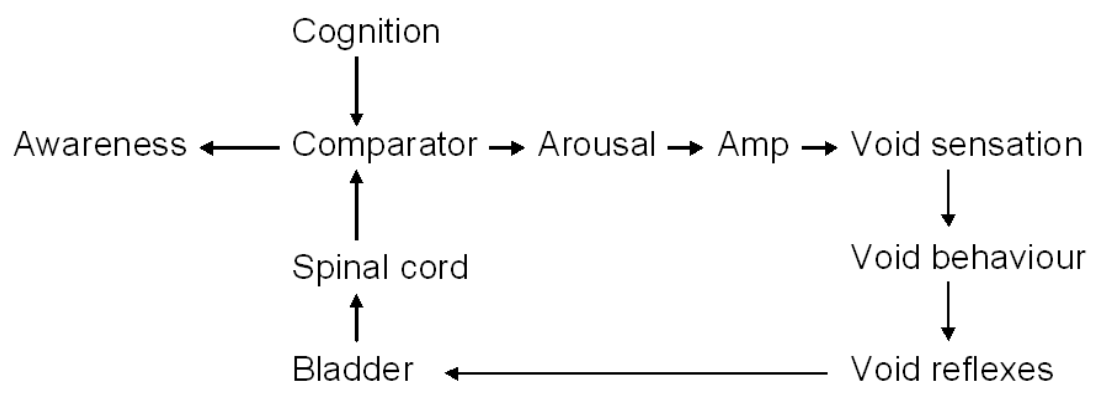




\section{Conclusions}

So, what might be considered to be a well researched and understood physiological system, is, in fact, poorly understood with potentially grave omissions. We currently cannot be certain that the information on bladder sensation derived from the different studies is what the subjects actually perceived or just merely what the observer wanted them to perceive and describe. We need to open discussion and research on the different topics described above and must find the right way forward. An "alternative" method to improve our knowledge could be focus group experiments during which healthy volunteers and patients discuss how bladder sensation develops, how and where the sensations are felt and which words are best suited to describe bladder sensation. Drawing "focused introspection" curves may be another non-invasive alternative to study the development of bladder sensation.

Urgency, frequency and incontinence, the Syndrome of the Overactive Bladder Symptom Complex, affects many people and decreases their quality of life. If we are to help such people we need a clear and fundamental understanding of the physiology, pharmacology and pathology of the bladder and all its complex and integrative functions such as bladder sensation. Furthermore it is essential that we have robust and simple terminology that is correctly interpreted by patients and physicians. Once this is established we need to develop our knowledge on what is going on in the periphery to generate and modulate sensation and what goes on in the CNS to process this information and drive voiding behaviour.

\section{References}

1. Fowler, C. J., Griffiths, D., de Groat, W. C.: The neural control of micturition. Nat Rev Neurosci, 9: 453, 2008

2. Abrams, P., Cardozo, L., Fall, M. et al.: The standardisation of terminology of lower urinary tract function: report from the Standardisation Sub-committee of the International Continence Society. Am J Obstet Gynecol, 187: 116, 2002

3. Gillespie, J. I., van Koeveringe, G. A., de Wachter, S. G. et al.: On the origins of the sensory output from the bladder: the concept of afferent noise. BJU Int, 103: 1324, 2009

4. Kanai, A., Andersson, K. E.: Bladder afferent signaling: recent findings. J Urol, 183: 1288

5. Xu, L., Gebhart, G. F.: Characterization of mouse lumbar splanchnic and pelvic nerve urinary bladder mechanosensory afferents. J Neurophysiol, 99: 244, 2008

6. Denny-Brown, D., Robertson, E. G.: On the physiology of micturition. Brain, 56: 149, 1933

7. Nathan, P. W.: Sensations associated with micturition. Br J Urol, 28: 126, 1956

8. Wyndaele, J. J.: The normal pattern of perception of bladder filling during cystometry studied in 38 young healthy volunteers. J Urol, 160: 479, 1998

9. Wyndaele, J. J., De Wachter, S.: Cystometrical sensory data from a normal population: comparison of two groups of young healthy volunteers examined with 5 years interval. Eur Urol, 42: 34, 2002

10. Chapple, C. R., Artibani, W., Cardozo, L. D. et al.: The role of urinary urgency and its measurement in the overactive bladder symptom syndrome: current concepts and future prospects. BJU Int, 95: 335, 2005

11. Erdem, E., Akbay, E., Doruk, E. et al.: How reliable are bladder perceptions during cystometry? Neurourol Urodyn, 23: 306, 2004

12. Erdem, E., Tunckiran, A., Acar, D. et al.: Is catheter cause of subjectivity in sensations perceived during filling cystometry? Urology, 66: 1000, 2005

13. De Wachter, S., Van Meel, T. D., Wyndaele, J. J.: Can a faked cystometry deceive patients in their perception of filling sensations? A study on the reliability of spontaneously reported cystometric filling sensations in patients with non-neurogenic lower urinary tract dysfunction. Neurourol Urodyn, 27: 395, 2008

14. Dompeyre, P., Fritel, X., Bader, G. et al.: Bladder sensitivity testing using a visual analogue scale: comparative cystometric study on women. Neurourol Urodyn, 26: 350, 2007

15. Morrison, J. F. B.: Sensations arising from the lower urinary tract. In: The physiology of the lower urinary tract. Edited by M. Torrens and J. F. B. Morrison: Springer-Verlag, pp. 89-131, 1987 
16. Riddoch, G.: Conduction of sensory impulses from the bladder by the inferior hypogastrics and the central afferent connections of these nerves. J Physiol, 54: 134, 1921

17. Gunterberg, B., Norlen, L., Stener, B.: Neurourologic evaluation after resection of the sacrum. Invest Urol, 13: 56, 1975

18. Wyndaele, J. J.: Investigating afferent nerve activity from the lower urinary tract: highlighting some basic research techniques and clinical evaluation methods. Neurourol Urodyn, 29: 56, 2010

19. Bahns, E., Ernsberger, U., Janig, W. et al.: Functional characteristics of lumbar visceral afferent fibres from the urinary bladder and the urethra in the cat. Pflugers Arch, 407: 510, 1986

20. Bahns, E., Halsband, U., Janig, W.: Responses of sacral visceral afferents from the lower urinary tract, colon and anus to mechanical stimulation. Pflugers Arch, 410: 296, 1987

21. Habler, H. J., Janig, W., Koltzenburg, M.: Activation of unmyelinated afferent fibres by mechanical stimuli and inflammation of the urinary bladder in the cat. J Physiol, 425: 545, 1990

22. Moss, N. G., Harrington, W. W., Tucker, M. S.: Pressure, volume, and chemosensitivity in afferent innervation of urinary bladder in rats. Am J Physiol, 272: R695, 1997

23. De Wachter, S., De Laet, K., Wyndaele, J. J.: Does the cystometric filling rate affect the afferent bladder response pattern? A study on single fibre pelvic nerve afferents in the rat urinary bladder. Neurourol Urodyn, 25: 162, 2006

24. Robertson, A. S., Griffiths, C. J., Ramsden, P. D. et al.: Bladder function in healthy volunteers: ambulatory monitoring and conventional urodynamic studies. Br J Urol, 73: 242, 1994

25. De Wachter, S., Wyndaele, J. J.: Frequency-volume charts: a tool to evaluate bladder sensation. Neurourol Urodyn, 22: 638, 2003

26. Naoemova, I., De Wachter, S., Wyndaele, J. J.: Comparison of sensation-related voiding patterns between continent and incontinent women: a study with a 3-day sensation-related bladder diary (SR-BD). Neurourol Urodyn, 27: 511, 2008

27. Naoemova, I., Van Meel, T., De Wachter, S. et al.: Does sensory bladder function during cystometry differ from that in daily life? A study in incontinent women. Neurourol Urodyn, 28: 309, 2009

28. Naoemova, I., De Wachter, S., Wuyts, F. L. et al.: Do sensation-related bladder diaries differ between patients with urodynamically confirmed and non-objectivised urinary incontinence? Int Urogynecol J Pelvic Floor Dysfunct, 19: 213, 2008

29. Digesu, G. A., Khullar, V., Panayi, D. et al.: Should we explain lower urinary tract symptoms to patients? Neurourol Urodyn, 27: 368, 2008

30. Digesu, G. A., Basra, R., Khullar, V. et al.: Bladder sensations during filling cystometry are different according to urodynamic diagnosis. Neurourol Urodyn, 28: 191, 2009

31. Blaivas, J. G., Panagopoulos, G., Weiss, J. P. et al.: Two types of urgency. Neurourol Urodyn, 28: 188, 2009

32. De Wachter, S., Hanno, P.: Urgency: all or none phenomenon? Neurourol Urodyn, 29: 616, 2010

33. Heeringa, R., De Wachter, S. G. G., van Kerrebroeck, P. E. V. A. et al.: Normal Bladder Sensations in Healthy Volunteers: A Focus Group Investigation. Neurourol Urodyn, in press

34. Naoemova, I., De Wachter, S., Wuyts, F. L. et al.: Reliability of the 24-h sensation-related bladder diary in women with urinary incontinence. Int Urogynecol J Pelvic Floor Dysfunct, 19: 955, 2008

35. Vaughan, C. W., Satchell, P. M.: Urine storage mechanisms. Prog Neurobiol, 46: 215, 1995 



\section{Degree of urge, perception of bladder fullness and bladder volume: how are they related?}

Rhea Heeringa ${ }^{1}$, Gommert A. van Koeveringe ${ }^{1,2}$, Bjorn Winkens ${ }^{3}$, Philip E.V. van Kerrebroeck ${ }^{1,2}$, Stefan G.G. de Wachter ${ }^{1,2}$

1. Department of Urology

Maastricht University Medical Centre; The Netherlands

2. European Graduate School of Neuroscience (EURON)

Maastricht University Medical Centre; The Netherlands

3. Department of Statistics and Methodology

Maastricht University Medical Centre; The Netherlands

Journal of Urology - 186 (4) : 1352-7, 2011

Epub: 2011 August 18 


\section{Abstract}

Purpose: This study evaluates the reliability of the degree of urge scale and perception of bladder fullness scale, the relationship between both scales and bladder volume and possible differences between bladder sensation experienced in a controlled or uncontrolled setting.

Materials and Methods: Volunteers and patients with overactive bladder symptoms participated in the protocols. They were asked to grade their bladder sensation on a 4 point urge scale ranging from 'no sensation' to 'need to void now' and assess the perception of fullness on a visual analogue scale ranging from 'empty' to 'full' bladder. This was assessed on a three day bladder diary and during a controlled non-invasive bladder filling.

Results: Both scales were reliable and were each significantly related to bladder volume $(p<0.001)$. The agreement between both scales and voided volume was poor. Patients and volunteers grade their bladder fullness and degree of urge to be higher in an uncontrolled setting than in a controlled setting.

Conclusions: The perception of bladder fullness and the degree of urge both have a significant relation with bladder volume, in controlled and uncontrolled settings. Because of the poor agreement between the scales and the actual voided volume these scales cannot replace measuring voided volumes. The perception of bladder sensation depends on the setting. Bladder sensations in the hospital might underestimate the bother of the symptoms a patient experiences in daily life. 


\section{Introduction}

A sensation is subjective and its evaluation is therefore difficult. However, several authors have proven that by means of an urgency scale bladder sensation before each void can be evaluated ${ }^{1-6}$. Although the urgency scale might differ between studies, all studies have shown a significant difference in voided volumes between the grades on an urgency scale, but on the other hand a big overlap in voided volumes between the different urgency grades. This suggests that the degree of urge might not always be correlated to bladder volume.

Recently a new method to study bladder sensation in a non-invasive manner is introduced. Through a water loading protocol and continuous introspection, subjects are able to draw their perceived sensation on a Visual Analogue Scale (VAS) representing the intensity of the sensation, ranging from empty bladder to full bladder ${ }^{7}$. How the VAS scale correlates with the urge/urgency scale and with the voided volume is currently unknown. Having a sensory scale that agrees well with the voided volume would be ideal because it can replace the measurement of all voided volumes which is quite a burden for some patients.

This study evaluates the relationships between the perception of bladder fullness (wordless scale), the urge scale and the voided volume by measuring these parameters on a sensation-related bladder diary (SR-BD). The reliability of the degree of urge scale and perception of bladder fullness scale and the relationship between both scales and bladder volume were assessed. Furthermore this study evaluates the relationship between bladder sensation experienced in a controlled and an uncontrolled setting by using both scales at home and in the hospital.

\section{Materials and methods}

The study consists of two separate protocols, both conducted in volunteers and patients with overactive bladder symptoms. All participants were recruited by advertisement. The local Medical Ethical Committee approved this study.

\section{Protocol 1: SR-BD}

To have data on the normal voiding and drinking behaviour of each participant, all participants filled out a three-day SR-BD with the VAS and 4 point scale to grade perception of bladder fullness and degree of urge, respectively. This was done before each controlled bladder filling sessions. The bladder fullness was graded on a VAS ranging from empty to full. The degree of urge scale was, in accordance with previous studies ${ }^{1,8}$, graded according to the following definitions: 0) no sensation 1) normal desire; voiding can be postponed for at least $30 \mathrm{~min}$. 2) strong desire; voiding can be postponed for about 10 min. 3) need to void now.

\section{Protocol 2: Controlled bladder filling sessions through rapid natural diu- resis}

All participants followed two non-invasive filling sessions in the hospital, with ten days interval. A constant high diuresis was aimed for by applying a water loading protocol 
which can be used to study bladder sensations non-invasively ${ }^{7}$. This water loading protocol was chosen aiming to reach maximum bladder capacity by natural diuresis over a period of about 1 hour. To start with an empty bladder, participants voided at the beginning of the session. During the session participants were regularly asked to grade their perception of bladder fullness on a VAS and grade their degree of urge on a four-point scale. The protocol ended with "need to void now" and voided volumes were measured. Because the induced diuresis is constant and the duration of the session for each participant is known, we were able to calculate the bladder volume at each time point.

\section{Statistical analysis}

Continuous variables were presented as means with standard deviations, categorical variables as frequency and percentage. For continuous variables, the differences between patients and volunteers or hospital and home were analyzed using independentsamples t-test or Mann Withney $U$ test where appropriate. For categorical variables, Chisquare, Fisher's exact test or Bowker's test of symmetry were used. Reliability of the evaluated parameters during filling sessions was calculated using intraclass correlation coefficient (ICC). Linear mixed models were used to evaluate the relation between either sensory scale with bladder volume and both sensory scales with each other. This model was used since it adjusts for correlated measurements within individuals. To evaluate if the information from a sensory measure can replace the observed voided volume, the agreement between observed and estimated bladder volume at home, obtained from linear mixed model with perception of bladder fullness and/or urge scale as explanatory variable(s), was evaluated by means of Bland and Altman plots. A p-value $\leq 0.05$ was considered as statistically significant. All analyses were performed using SPSS $₫ 18$.

\section{Results}

Twelve patients (mean age 62.3 year; 8 male) and 26 volunteers (mean age 39.7 year; 9 male) participated in this study.

\section{Sensation related bladder diary}

The total number of voids fully registered as requested (voided volume, perception of fullness and degree of urge) for patients was 701 (99.6\% of all voids) and for volunteers 930 (98.8\% of all voids). Detailed information on the voids and the differences between study groups are found in table 1. 
Table 1 shows detailed information on the data of the sensation related bladder diary for both groups: the twelve OAB patients $(p)$ and twenty-six volunteers $(v)$.

* significant difference between patients and volunteers with $p<0.001$

a) Average value within a person over all days or voids.

b) Mode within a person, i.e. most frequent degree of urge, over all voids.

\begin{tabular}{|l|l|l|l|l|l|l|l|l|l|l|l|}
\hline & \multicolumn{2}{|l|}{$\begin{array}{l}\text { Frequency per } \\
\text { day* }\end{array}$} & \multicolumn{2}{l|}{$\begin{array}{l}\text { Voided volume } \\
(\mathrm{ml})^{*} \text { a) }\end{array}$} & \multicolumn{2}{l|}{$\begin{array}{l}\text { Perception of } \\
\text { bladder fullness }\end{array}$} \\
\end{tabular}

\section{The urge scale and perception of fullness VAS scale}

Reliability of the urge scale and the perception of fullness VAS scale was tested by means of the ICC between the first 3 and second 3 days. Coefficients for the mean voided volumes at the different grades of urge and perception of fullness can be found in table 2. Except for the ICC with perception of fullness score 1 and degree of urge score 0 , the coefficients were above 0.7 , which means good to excellent reliability ${ }^{8}$.

The perception of bladder fullness and the urge scale were significantly related to each other $(p=0.019)$ (figure 1). There was a positive correlation between the sensory measures on both scales and bladder volume both for patients and volunteers, and for the measurements at home and in the hospital (all $p<0.001)$. Furthermore, the linear mixed models showed that both scales were independently related to bladder volume, meaning that they gave additional information on the voided volume.

Bland-Altman plots showed that the differences between observed bladder volume and estimated bladder volume from bladder fullness ranged from -321 to $448 \mathrm{ml}$, indicating poor agreement for bladder fullness in both groups. The same was found for degree of urge with differences ranging from -346 to $514 \mathrm{ml}$. If both scales were used to estimate the bladder volume the differences with observed volumes ranged from -288 to $420 \mathrm{ml}$. So even if both scales are used simultaneously, there is a poor agreement between the scales and bladder volume. 
Table 2 shows the ICC for the mean voided volumes at the different grades of both scales.

\begin{tabular}{|c|c|c|c|c|c|c|c|c|c|c|}
\hline & \multicolumn{10}{|c|}{ Perception of bladder fullness } \\
\hline & 1 & 2 & 3 & 4 & 5 & 6 & 7 & 8 & 9 & 10 \\
\hline \multirow[t]{3}{*}{ ICC } & 0.14 & 0.80 & 0.71 & 0.87 & 0.83 & 0.96 & 0.86 & 0.85 & 0.87 & 0.86 \\
\hline & \multicolumn{10}{|c|}{ Degree of urge } \\
\hline & 0 & & & & & 2 & & 3 & & \\
\hline ICC & 0.58 & & & & & 0.94 & & & & \\
\hline
\end{tabular}

\section{Comparing home and hospital sensory data}

For the same degree of urge (figure 1) and the same bladder volume (figure 2), patients and volunteers graded their bladder fullness higher at home than during the physiological filling session in the hospital (see also table 3). For the same bladder volume the degree of urge is also graded higher at home than during the filling session.

Figure 1 shows the relationship between the degree of urge scale (x-axis) and the perception of fullness (y-axis) for patients $(A)$ and volunteers (B).
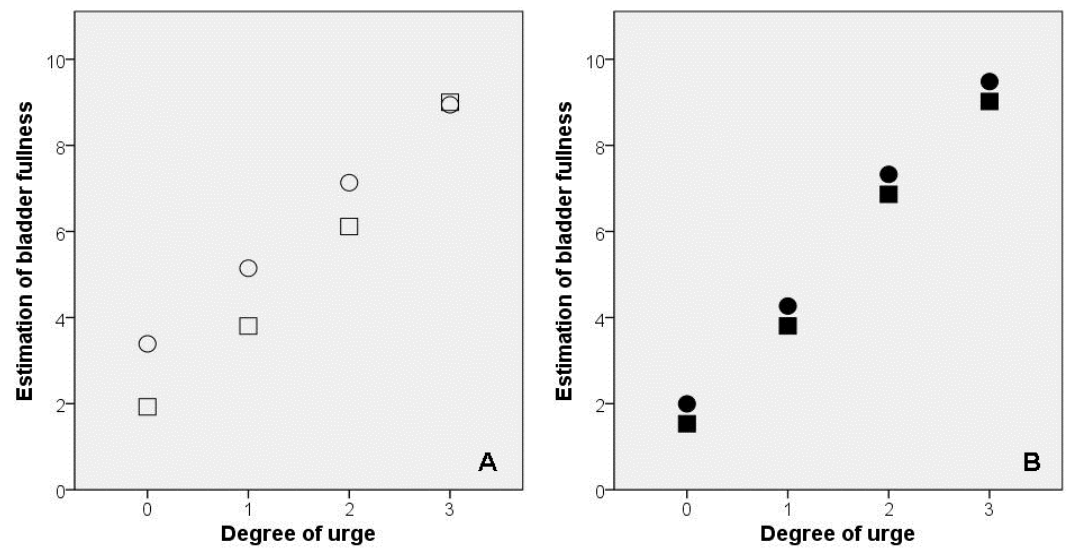
Degree of urge, perception of bladder fullness and bladder volume: how are they related?

Figure 2 shows the relation between the bladder volume and the perception of bladder fullness for one patient. It can be seen that for the same bladder volume the perception of bladder fullness is higher at home than during the session. For example: a bladder volume of $200 \mathrm{ml}$ is estimated to have a fullness at home ranging from 5 to 8 while this same bladder volume is estimated with a fullness around 3,5 during the session in the hospital.

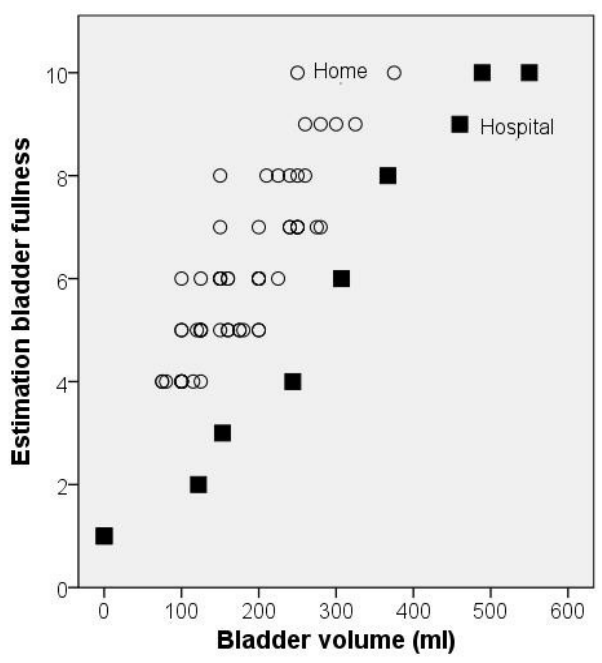

Table 3 shows detailed information on the differences between measurements at home and during the controlled sessions in the hospital for the perception of bladder fullness and the degree of urge. $P$ values represent the difference between both locations.

* significant difference between both locations with $p<0.001$

\# no significant difference between locations with $p=0.088$

a) For each person, the average value was taken over all voids.

${ }^{b)}$ Mode within a person, i.e. most frequent degree of urge, over all voids.

\begin{tabular}{|c|c|c|c|c|c|c|c|c|c|c|}
\hline & \multicolumn{2}{|c|}{$\begin{array}{l}\text { Perception of } \\
\text { bladder fullness }^{\text {a) }}\end{array}$} & \multicolumn{8}{|c|}{ Degree of urge $^{b)}$} \\
\hline & \multicolumn{2}{|c|}{ Mean (SD) } & \multicolumn{8}{|c|}{ Frequency (\% within group) } \\
\hline & \multirow[t]{2}{*}{ Patient $^{*}$} & \multirow{2}{*}{$\begin{array}{l}\text { Volun- } \\
\text { teer* }\end{array}$} & \multicolumn{4}{|c|}{ Patient ${ }^{\#}$} & \multicolumn{4}{|c|}{ Volunteer * } \\
\hline & & & 0 & 1 & 2 & 3 & 0 & 1 & 2 & 3 \\
\hline $\begin{array}{l}\text { Hospi- } \\
\text { tal }\end{array}$ & $\begin{array}{l}4.3 \\
(1.5)\end{array}$ & $\begin{array}{l}4.3 \\
(1.1)\end{array}$ & $\begin{array}{l}7 \\
(58.3)\end{array}$ & $\begin{array}{l}1 \\
(8.3)\end{array}$ & $\begin{array}{l}4 \\
(33.3)\end{array}$ & 0 & $\begin{array}{l}24 \\
(92.3)\end{array}$ & $\begin{array}{l}2 \\
(7.7)\end{array}$ & 0 & 0 \\
\hline Home & $\begin{array}{l}7.8 \\
(1.7)\end{array}$ & $\begin{array}{l}6.0 \\
(1.1)\end{array}$ & 0 & 0 & $\begin{array}{l}5 \\
(41.7)\end{array}$ & $\begin{array}{l}7 \\
(58.3)\end{array}$ & $\begin{array}{l}2 \\
(7.7)\end{array}$ & $\begin{array}{l}16 \\
(61.5)\end{array}$ & $\begin{array}{l}7 \\
(26.9)\end{array}$ & $\begin{array}{l}1 \\
(3.8)\end{array}$ \\
\hline $\mathbf{P}$ & $<0.001$ & $<0.001$ & \multicolumn{4}{|c|}{$=0.088$} & \multicolumn{4}{|c|}{$<0.001$} \\
\hline
\end{tabular}




\section{Discussion}

Bladder sensations are subjective and therefore hard to evaluate. Increased and decreased bladder sensation are important symptoms and signs in several lower urinary tract dysfunctions ${ }^{9,10}$. It is therefore necessary to understand how sensation develops and how it can be correctly quantified. Sensory evaluation during cystometry is the method mostly studied, but it is invasive and occurs in laboratory settings. The sensation-related bladder diary (SR-BD) assesses bladder sensation at each micturition, noninvasively and at home. This method is recommended in the workup of patients with lower urinary tract dysfunctions. Subjects are asked to measure the voided volume with a measuring cup and grade the desire to void according to a predefined scale, which can differ between studies ${ }^{1-3,11}$. In practice, some subjects find it difficult to harmonize these volume measurements with their professional and social life. Having a sensory scale that correlates well with the voided volume could replace the need to measure all voided volumes and would therefore be ideal, as also proposed by others ${ }^{3}$. In addition one might expect future treatment options for the $O A B$ syndrome that might reduce the bother for the patient while a significant change in voided volume or frequency of voiding is difficult to show. In these cases this assessment might prove to be a useful tool to evaluate bladder sensation.

The results on the SR-BD are, for patients as well as volunteers, comparable with findings from other studies ${ }^{1,2,4,5}$. Therefore, despite the age difference between both groups, we think it is fair to conclude that our younger volunteer group is comparable with older volunteer groups and can therefore be used as a control group for our patient population.

It has been shown repeatedly that the mean voided volumes are significantly different between different grades and there is a positive correlation between voided volume and grade of desire to void $^{1,5,12}$. In addition, however there also appears to be a large range in bladder volumes per grade of desire to void and a substantial overlap in volumes between different grades ${ }^{5,8}$. Brain imaging studies have shown that different brain regions are involved in registering bladder filling/volume and perceiving bladder sensation and that these regions can be independently disturbed or modulated ${ }^{13-15}$. This suggests that the relation between bladder volume and bladder sensation is more complex than a simple linear relation.

Neurophysiological studies in animals have shown that afferent activity is send up the spinal cord to the brain stem, where bladder filling/volume is continuously measured ${ }^{16}$. From that point of view, the ideal scale to grade bladder volume would be a continuous one. Therefore a continuous VAS scale for bladder fullness was chosen as a measure the bladder volume in this study. This measure was then linked to measured voided volumes and the established urge scale was used as a measure for bladder sensation. Although one could argue that these measures make the bladder diary complicated and difficult to use, with good written and oral instructions almost every void in this study was registered as requested on all three scales. Furthermore the reliability of both the urge measure and fullness VAS measure is good to excellent when looking at the intraclass correlation coefficients, except for the voids graded as almost empty (fullness 1 ) and without sensation (degree of urge 0 ). These values are in agreement with the reliability 
measures of the urge scale previously published in patients with urgency, stress and mixed urinary incontinence ${ }^{8}$.

In agreement with previous studies ${ }^{1,5}$, our data show that both the perception of bladder fullness and the urge scale measures are positively correlated with bladder volume. However, the Bland and Altman analysis shows that for the individual measurements the agreement between the sensory measure (urge and fullness) and voided volume is poor, with volumes ranging up to $800 \mathrm{ml}$ for a single urge scale or fullness measure. These data clearly show that neither the established urge scale nor the continuous perception of fullness VAS, nor even a combination of both scales can replace the measurement of the voided volume. The use of the combination of both scales may however help to differentiate patients with $\mathrm{OAB}$ complaints, as both scales are independently related to volume. This suggests that they provide different and complementary information on how persons deal with bladder volume. For example, the combination of a voided volume of $20 \%$ of the maximum bladder capacity, urgent desire to void (urge scale 3 ) and nearly empty bladder (VAS fullness scale 1) may point to a "central" cause such as emotional disturbances or nervousness providing a different perceptual threshold, whereas the voided volume of $20 \%$ of the maximum bladder capacity, urgent desire to void (urge scale 3) with a full bladder (VAS fullness scale 9) may point to a "peripheral" bladder disorder such as detrusor over activity. This hypothesis is based on individual readings of a small group of patients and needs to be studied in a larger patient group.

This study also found that there is a difference in how subjects experience sensations in a controlled hospital setting and an uncontrolled setting at home. Both volunteers and patients experience their perception of bladder fullness and degree of urge, for the same bladder volume, to be higher at home than during the filling session in the hospital. Recently the possibility of a central modulation of sensory information was introduced ${ }^{17}$. This concept entails that sensations generated during a controlled bladder filling are continuous and form the basic pattern of perception of bladder sensation. However, when making the decision to void in an uncontrolled setting, this pre-void sensation may be amplified centrally and made more significant to the observer. This could explain why we found a difference in both study settings. Another possible explanation is the fact that during the session all subjects were aware that their bladder was becoming full, they were asked to postpone voiding as long as possible and graded their sensation during one bladder filling on a regular basis. At home a sensation from the bladder, of either intensity, might sooner interfere with activities and therefore the bladder might be classified fuller than during the session. Final explanations for this difference might be water load protocol used in the controlled setting or the possibility that during the hospital session patients and volunteers are in a less relaxed state and therefore have a higher basic tension of their pelvic floor. This effect might trigger the detrusor inhibition reflex or guarding reflex and so increase the detected bladdervolumes ${ }^{18}$.

That sensations at home are different from sensations experienced in the hospital has been shown before. Naoemova et al. showed that incontinent subjects experience different degrees of desire to void at larger volumes during cystometric bladder filling than in daily life ${ }^{12}$ and Yoon et al. showed that maximum cystometric bladder capacity is significantly smaller than maximum environmental voided volumes ${ }^{19}$. Furthermore Robertson 
and colleagues found that there are significant differences between conventional artificial filling cystometry and ambulatory monitoring in healthy subjects ${ }^{20}$.

This study has the limitation that while comparing bladder sensation at home and at the hospital two different techniques are being used, respectively only grading sensation before a void and regularly grading sensation during a rapid physiological bladder filling. This study was not designed to specifically evaluate this difference in effect. Though analysis showed no difference between sex, we believe that the groups after subdivision are to small to draw hard conclusions. Recruiting patient by means of advertisement may induce a selection bias, by recruiting patients with more symptoms. However it was made clear to all patients that participating in this study would not influence their symptoms while we did not evaluate a treatment.

\section{Conclusion}

The degree of urge and the perception of bladder fullness are reliable to use and both scales have a significant relation with bladder volume in a controlled and uncontrolled setting. The combination of the perception of fullness scale and the degree of urge scale for a given void provides more information than either scale on its own but usage of these scales cannot replace measuring voided volumes. Bladder sensation in a uncontrolled setting is different from bladder sensation in a controlled setting and might be more dependent on influences from within or outside of the subject.

\section{References}

1. De Wachter, S., Wyndaele, J. J.: Frequency-volume charts: a tool to evaluate bladder sensation. Neurourol Urodyn, 22: 638, 2003

2. Honjo, H., Kawauchi, A., Ukimura, O. et al.: Analysis of bladder diary with urinary perception to assess overactive bladder symptoms in community-dwelling women. Neurourol Urodyn, 2009

3. Cartwright, R., Panayi, D., Cardozo, L. et al.: Reliability and normal ranges for the Patient's Perception of Intensity of Urgency Scale in asymptomatic women. BJU Int, 105: 832, 2009

4. Fitzgerald, M. P., Ayuste, D., Brubaker, L.: How do urinary diaries of women with an overactive bladder differ from those of asymptomatic controls? BJU Int, 96: 365, 2005

5. Naoemova, I., De Wachter, S., Wyndaele, J. J.: Comparison of sensation-related voiding patterns between continent and incontinent women: a study with a 3-day sensation-related bladder diary (SR-BD). Neurourol Urodyn, 27: 511, 2008

6. Blaivas, J. G., Panagopoulos, G., Weiss, J. P. et al.: The urgency perception score: validation and test-retest. J Urol, 177: 199, 2007

7. Heeringa, R., van Koeveringe, G. A., van Kerrebroeck, P. E. V. A. et al.: Forced diuresis as a tool for the noninvasive evaluation of bladder sensation. Neurourol Urodyn, 29: 909, 2010

8. Naoemova, I., De Wachter, S., Wuyts, F. L. et al.: Reliability of the 24-h sensation-related bladder diary in women with urinary incontinence. Int Urogynecol J Pelvic Floor Dysfunct, 19: 955, 2008

9. Nathan, P. W.: Sensations associated with micturition. Br J Urol, 28: 126, 1956

10. DasGupta, R., Fowler, C. J.: The management of female voiding dysfunction: Fowler's syndrome -- a contemporary update. Curr Opin Urol, 13: 293, 2003

11. Digesu, G. A., Basra, R., Khullar, V. et al.: Bladder sensations during filling cystometry are different according to urodynamic diagnosis. Neurourol Urodyn, 28: 191, 2009

12. Naoemova, I., Van Meel, T., De Wachter, S. et al.: Does sensory bladder function during cystometry differ from that in daily life? A study in incontinent women. Neurourol Urodyn, 28: 309, 2009

13. Griffiths, D., Tadic, S. D.: Bladder control, urgency, and urge incontinence: evidence from functional brain imaging. Neurourol Urodyn, 27: 466, 2008

14. Blok, B. F.: Central pathways controlling micturition and urinary continence. Urology, 59: 13, 2002

15. Blok, B. F., Willemsen, A. T., Holstege, G.: A PET study on brain control of micturition in humans. Brain, 120 ( Pt 1): 111, 1997 
Degree of urge, perception of bladder fullness and bladder volume: how are they related?

16. Shea, V. K., Cai, R., Crepps, B. et al.: Sensory fibers of the pelvic nerve innervating the Rat's urinary bladder. J Neurophysiol, 84: 1924, 2000

17. Gillespie, J. I., van Koeveringe, G. A., de Wachter, S. G. et al.: On the origins of the sensory output from the bladder: the concept of afferent noise. BJU Int, 103: 1324, 2009

18. van Koeveringe, G. A., Rahnama'i, M. S., Berghmans, B. C.: The additional value of ambulatory urodynamic measurements compared with conventional urodynamic measurements. BJU Int, 105: 508

19. Yoon, E., Swift, S.: A comparison of maximum cystometric bladder capacity with maximum environmental voided volumes. Int Urogynecol J Pelvic Floor Dysfunct, 9: 78, 1998

20. Robertson, A. S., Griffiths, C. J., Ramsden, P. D. et al.: Bladder function in healthy volunteers: ambulatory monitoring and conventional urodynamic studies. Br J Urol, 73: 242, 1994 



\title{
'Focused introspection' during naturally increased diuresis: description and repeatability of a new method to study bladder sensation non-invasively
}

\author{
R. Heeringa ${ }^{1}$, S. G. G. de Wachter ${ }^{1,2}$, G. A. van Koeveringe ${ }^{1,2}$, B. Winkens ${ }^{3}$, \\ P. E. V. A. van Kerrebroeck ${ }^{1,2}$, J. I. Gillespie ${ }^{4}$ \\ 1. Department of Urology \\ Maastricht University Medical Centre; The Netherlands \\ 2. European Graduate School of Neuroscience (EURON) \\ Maastricht University; The Netherlands \\ 3. Department of Statistics and Methodology \\ Maastricht University; The Netherlands \\ 4. Uro-physiology Research Group \\ Medical and Dental School, University of Newcastle Upon Tyne, England
}

Neurourology and Urodynamics - Submitted 


\begin{abstract}
Introduction: Bladder sensation is currently studied by means of cystometry or sensationrelated bladder diaries. Both have important disadvantages, for example being invasive or not describing the entire pattern of bladder filling. This study describes a new noninvasive method to study the origin and development of bladder filling sensation and evaluates the repeatability of the method.
\end{abstract}

Method: In total eighteen volunteers participated in the study and were given a water loading protocol consisting of $1000 \mathrm{ml}$ water intake one hour before the session started and $200 \mathrm{ml}$ every 10 minutes during the session, to ensure a constant high diuresis. To evaluate diuresis rate, 7 of them were asked to void every 15 minutes in a voiding cup and the volumes were measured. The other 11 volunteers participated in a different protocol were they graded bladder sensation on regular time points, on an empty graph with time on the $\mathrm{X}$-axis and intensity of sensation on the $\mathrm{Y}$-axis. The protocol ended with an absolute need to void and voided volumes were measured. This protocol was conducted three times with a 10 days interval.

Results: For each subject the increased diuresis was not different between the sessions. Two patterns of developing sensation were found. One consisted of a silent phase without any sensation, followed by a slow increase in sensation intensity. Finally, there was a steep rise in the intensity of sensation which immediately led to an absolute need to void (type A). A second pattern was observed which was similar to type A but which had a flattening of the sensation curve prior to the sensation of an absolute need to void (type B). For each individual the pattern was constant during the three sessions.

Conclusion: A water loading protocol, inducing a constant high diuresis, allows the evaluation of bladder sensations during a non-invasive controlled filling. The absolute need to void can develop in two different patterns, but the pattern is constant intra-individually. 


\section{Introduction}

Adequate perception of bladder filling enables us to perform our daily tasks without continuous intrusions from desire to void. Healthy persons can therefore, seemingly unconsciously, integrate bladder emptying in their professional and social life. Disturbances in the generation or interpretation of this 'bladder information' may lead to urinary tract dysfunctions with an impact on health and quality of life. Increased perception of bladder signals may give rise to frequency, urgency and even incontinence, whereas decreased perception may lead to impaired bladder emptying, urinary tract infection and even kidney failure ${ }^{1}$. Adequate and reproducible tests are mandatory to make a correct diagnosis of any sensory disturbance in the lower urinary tract in order to optimize diagnosis and patient treatment.

Different methods have been described to measure or evaluate bladder sensation. Since sensation is subjective, the methods for evaluating bladder sensation have been the subject of many debates. Cystometric bladder filling is used to evaluate bladder sensation and has been shown to be a reproducible method to evaluate the normal and pathological pattern of bladder filling sensation ${ }^{2}$. The reproducibility seems to depend on the information or terminology provided to the subject. Furthermore, a cystometric measurement requires placement of a transurethral catheter and retrograde bladder filling which may also influence the outcome of investigation. This may explain why the results from different research groups appear to contradict ${ }^{3-6}$. The use of a bladder diary has been proposed as a non-invasive tool to study bladder sensation ${ }^{7}$. For this, patients are asked to grade their perception of bladder filling just prior to a void, and this sensory grade is then linked to the voided volume. Differences between volunteers and patients with stress incontinence and/or urgency incontinence have been described ${ }^{8}$, and different scales have been used in clinical trials assessing drug effects on overactive bladder symptoms and bladder sensation ${ }^{9,10}$. However, strictly speaking, the "sensation related bladder diary" assesses the bladder sensation a person has at the time he/she decides to go to void. The assessment does not give any information on the development and changes in progression of bladder sensation.

The use of electrical stimulation with the electrical perception threshold has been advocated as a semi-objective method for sensory evaluation. Electrical stimulation is delivered through a transurethral catheter that enables a separate evaluation of the bladder and urethral perception threshold. However its role appears to be limited because there is no correlation with bladder filling sensation ${ }^{11}$ which we use to guide when and where to empty our bladder.

If we are to increase our knowledge on sensory lower urinary tract disorders, we need insights into how sensation develops and progresses to desire to void or urgency. This study describes and evaluates the repeatability of a new non-invasive method to assess the development of bladder sensation using a water loading protocol to induce a constant high diuresis.

\section{Materials and methods}

Healthy young volunteers recruited by advertisement participated in the study. To eliminate the need for transurethral catheterization to fill the bladder, a water loading protocol 
was designed, aiming at a constant high diuresis and a bladder filling that would lead to the sensation of a full bladder within 60-90 minutes. The water loading protocol consisted of $1000 \mathrm{ml}$ water intake one hour before the start of the session, with addition of $200 \mathrm{ml}$ every 10 minutes during the session. A total of $2000 \mathrm{ml}$ water intake was set as upper limit.

Protocol 1 was setup to evaluate the diuresis variation induced by the water loading protocol during the expected time span of the study protocol. Four female and three male volunteers with a mean age of $33 \pm 9$ years participated. At the start of the session all participants voided the bladder to completion (t0). Every 15 minutes they voided in a measuring cup, regardless of their bladder sensation (t15, t30, t45, t60) and the diuresis was calculated (voided volume $(\mathrm{ml}) / 15 \mathrm{~min}$ ). The protocol ended after 60 minutes. This protocol was done twice in every volunteer with 1 to 3 days interval. The variation in diuresis within a session and the reproducibility between the sessions was evaluated using Wilcoxon signed rank test with Bonferroni correction.

Protocol 2 was conducted in 7 female and 4 male volunteers with mean age of $22 \pm 3$ years, and was conducted three times with 10 days intervals. The water loading protocol was initiated one hour before the start of this "focused introspection" session. At the start of the session the subjects voided to completion to ensure an empty bladder. The subjects were then asked to note down on a graph the intensity of the bladder filling sensation they perceived every 10 minutes, complementary to noting down whenever they noticed any change. The graph provided to the subjects (figure 1) has an open-end time scale on the $\mathrm{X}$-axis and intensity of bladder fullness sensation ranging from 0 (no bladder sensation) to 100 (absolute need to void). The protocol ended when the subject indicated an absolute need to void. At that point they were allowed to void and the voided volume was measured. During the session, no information was given to what they might perceive during bladder filling, nor were they asked to describe or provide any terms related to bladder filling.

\section{Results}

\section{Protocol 1}

The mean diuresis for session 1 and 2 respectively was $13 \pm 6 \mathrm{ml} / \mathrm{min}$ and $14 \pm 6 \mathrm{ml} / \mathrm{min}$. The individual data for all participants is given in table 1. During a session, the diuresis was not different and showed no variation over the studied time period within an individual $(p=0.194)$. However the range in diuresis between individuals was large from 7 $\mathrm{ml} / \mathrm{min}$ to $24 \mathrm{ml} / \mathrm{min}$, despite the same water loading protocol.

\section{Protocol 2}

The mean diuresis over the whole session was $12 \pm 3 \mathrm{ml} / \mathrm{min}$. There was a large variation inter-individually (range $4-18 \mathrm{ml} / \mathrm{min}$ ), but intra-individually there was no difference between the three session ( $p>0.12$ ). The mean duration of a session was $62 \pm 12$ min (range: $38-86 \mathrm{~min}$ ), and was not different between the different sessions $(p>0.17)$. 
'Focused introspection' during a naturally increased diuresis: description and repeatability of a new method to study bladder sensation non-invasively

Table 1 Individual detailed diuresis rates during the two sessions and the different time points.

\begin{tabular}{|l|c|c|c|c|c|c|c|c|}
\hline & Session 1 & & & & Session 2 & & & \\
\hline Participant & $\mathrm{t} 15$ & $\mathrm{t} 30$ & $\mathrm{t} 45$ & $\mathrm{t} 60$ & $\mathrm{t} 15$ & $\mathrm{t} 30$ & $\mathrm{t} 45$ & $\mathrm{t} 60$ \\
\hline & 23 & 23 & 22 & 23 & 24 & 23 & 22 & 24 \\
\hline 2 & 9 & 9 & 9 & 9 & 10 & 10 & 11 & 11 \\
\hline 3 & 11 & 11 & 11 & 11 & 12 & 11 & 9 & 10 \\
\hline & 10 & 11 & 10 & 10 & 14 & 12 & 12 & 13 \\
\hline 5 & 7 & 7 & 7 & 9 & 10 & 10 & 10 & 10 \\
\hline & 7 & 10 & 10 & 10 & 13 & 10 & 13 & 10 \\
\hline Mean & 23 & 21 & 21 & 21 & 23 & 23 & 20 & 21 \\
\hline Stand Dev & 13 & 13 & 13 & 13 & 15 & 14 & 14 & 14 \\
\hline \multicolumn{2}{|l|}{ Data are diuresis rates in $\mathrm{ml} / \mathrm{min}$} & 6 & 6 & 6 & 6 & 6 & 5 & 6 \\
\hline
\end{tabular}

Typical examples of "focused introspection" curves are shown in figure 2. In 7 participants the curve was convex (left), whereas in 4 individuals, the curve was S-shaped (right). Initially, there is a silent phase (phase 1) during which bladder intensity remains 0 . At a certain point a first sensation is felt (sensation intensity rises above $0 \%$ ) and bladder sensation intensity rises slowly (phase 2). A third phase is defined as the point at which the slope of the curve increases and sensation intensity increases rapidly. For the convex curves, this steep rise (phase 3 ) leads to an absolute need to void $(100 \%)$, whereas for the S-shaped curves, a fourth phase can be identified, characterized by a decrease in slope of the curve and sensation intensity that increases at a lower rate (phase 4). This ultimately also leads to an absolute need to void.

No statistical difference was found between the three sessions in the volume at which a first sensation was noted ( $p>0.06$ ), but there was a large variation within an individual. Mean volumes respectively were $152 \pm 174 \mathrm{ml}, 231 \pm 156 \mathrm{ml}$ and $274 \pm 152 \mathrm{ml}$, for the first, second and third session. The slope of phase 2 and 3 , which represents the velocity of sensation intensity changes, showed less variation and was not different for the three sessions $(p>0.07)$. The mean voided volume at "absolute need to void" was $746 \pm 223$ $\mathrm{ml}$ (range: $240-1120 \mathrm{ml}$ ) and was not different between the three sessions $(p=0.99)$. Furthermore, the Area Under the Curve (AUC), which is used as quantitative measure for bladder intensity integrated for the whole curve, was not different for the three sessions (Friedman test, $p=0.09$ ). The "focused introspection" curves from the first session showed the least agreement with session 2 and 3 with the intraclass coefficients for AUC respectively 0.6 and 0.7 . The curve of session 2 showed an excellent agreement with session 3 , with an intraclass coefficient of 0.9 . 


\section{Chapter 4}

Figure 1 shows the "focused introspection" graphs. The volunteers were asked to mark with an X the intensity of sensation every 10 minutes or whenever they felt a change in sensation. Figure 1a shows an empty graph as given to the participants. Figure $1 \mathrm{~b}$ shows a graph as filled in by a participant. Figure $1 \mathrm{c}$ shows the graph used to analyse the data. Based upon the time and voided volume at absolute need $(100 \%)$ the bladder volumes are recalculated. To analyse the shape of the curve, the individual marks are connected by a straight line.
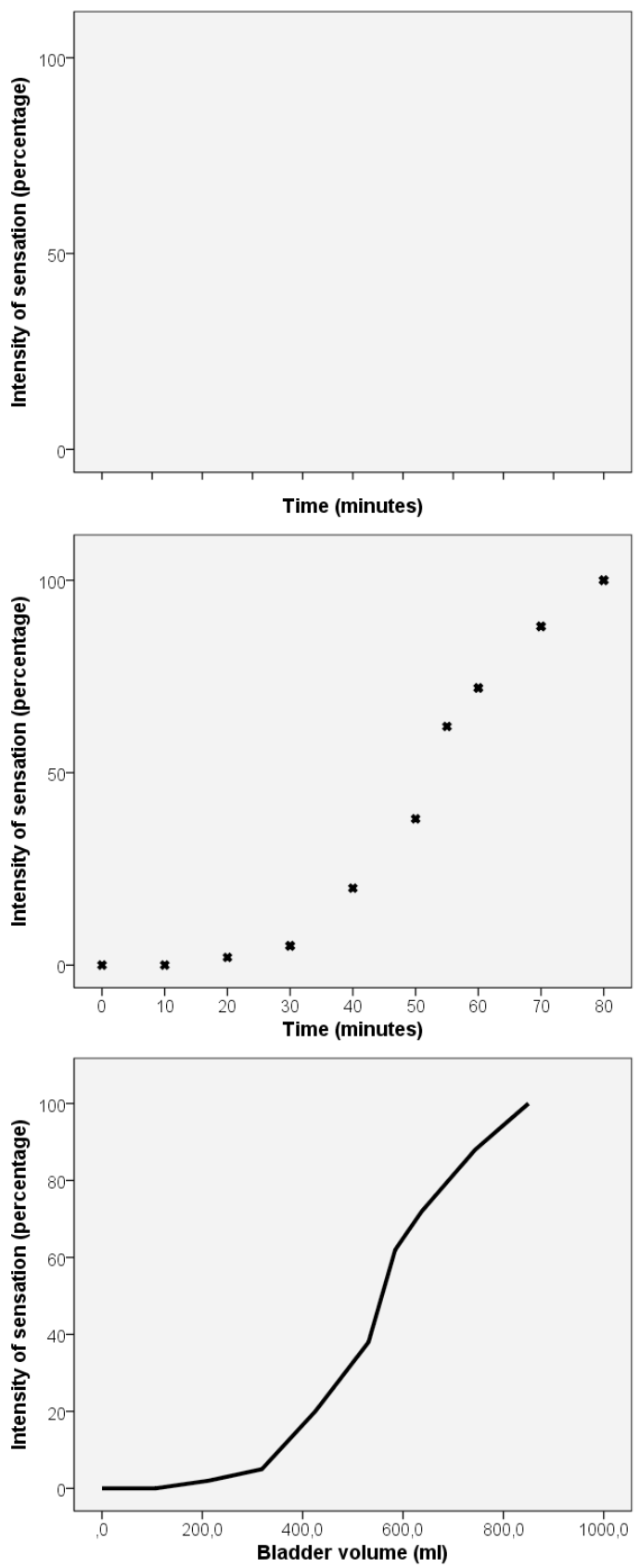
Figure 2 shows the two type of curves (A and B) with the phases (p) of each type. The first sensation of filling is indicated by FS. The absolute need to void is indicated by AN.
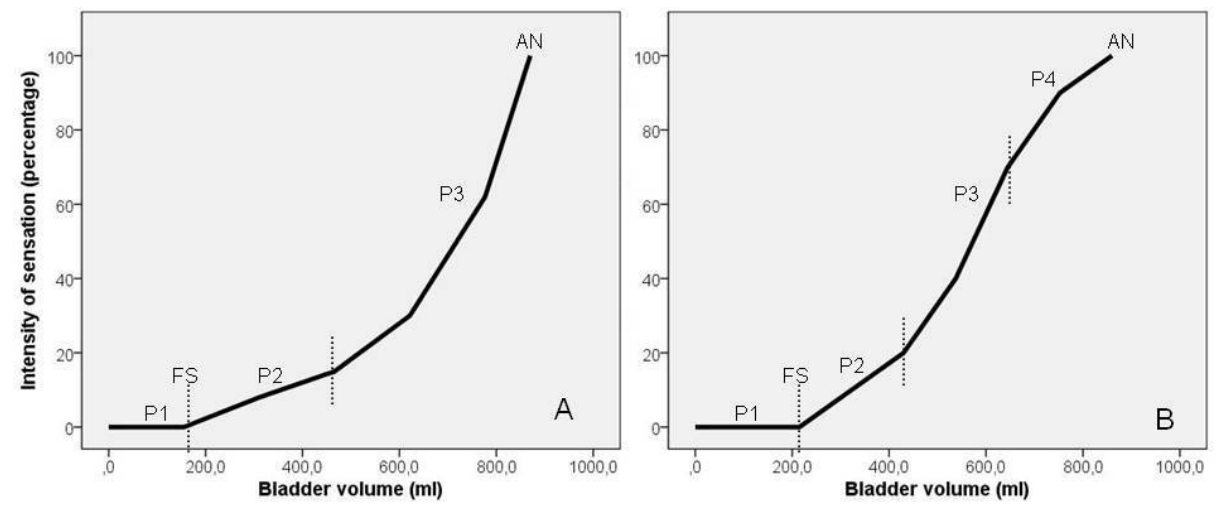

Figure 3 shows the curves for the three sessions for 2 different participants. Mark the difference in volume between participants.
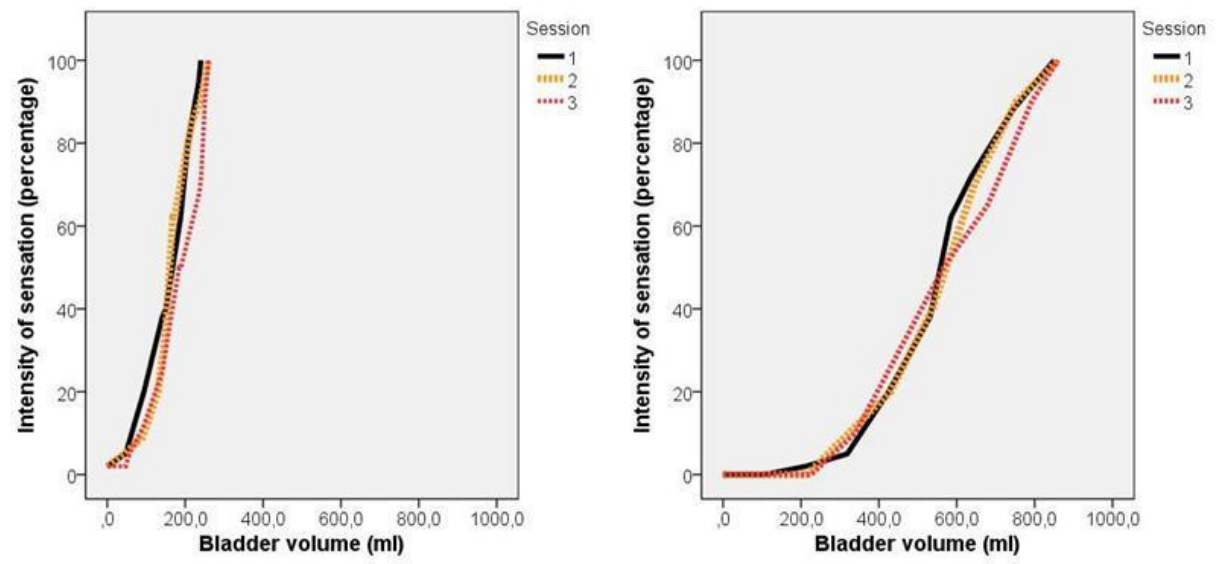

\section{Discussion}

This study presents and evaluates the reproducibility of a new method to study the development of bladder sensation non-invasively. Disturbed bladder sensation is increasingly acknowledged as the "primum movens" in the overactive bladder syndrome ${ }^{12}$ and is considered a contributing factor in the pathophysiology of bladder hypo-contractility and impaired bladder emptying ${ }^{1}$. In order to understand the pathophysiological mechanisms in these dysfunctions and optimize treatments, it is necessary to understand how sensation develops. Therefore reliable and reproducible tests to measure sensation are mandatory. Cystometric bladder filling has been proposed as a method to study bladder sensation adequately ${ }^{13,14}$. Although its reproducibility has recently been claimed ${ }^{2}$, it does seem to depend on the information or terminology provided to the subjects ${ }^{3-5}$. Furthermore subjects are catheterized and the bladder is not-physiologically filled. These factors 
may explain the debates on its reliability and reproducibility ${ }^{3-6}$. In order to avoid the influence of these "confounding" factors, we developed a water loading protocol to induce a constant high diuresis for bladder filling, aiming to have the sensation of an absolute need to void within a 60-90 minutes time span. Furthermore terms and words to describe bladder sensation were avoided. Instead sensation intensity was graded on a graph with absolute need to void as maximal (100\%) intensity.

None of the participants found the high water volume they had to drink in the proposed timeframe, as described in the water loading protocol, uncomfortable to do. Following this protocol, a constant high diuresis was induced during the next 60 minutes, with an average of $13-14 \mathrm{ml} / \mathrm{min}$. These values are significantly lower than the $30-50 \mathrm{ml} / \mathrm{min}$ used for cystometric bladder filling ${ }^{13,15}$. The lower filling rate induced by the water loading protocol may be important for a normal development of sensation, as nonphysiologically fast filling rates induce different afferent responses ${ }^{16}$ and change the volume at which the bladder is considered full ${ }^{15}$. Although the diuresis was constant within an individual, our results show a large variation between individuals with values ranging from 6 to $24 \mathrm{ml} / \mathrm{min}$. Diuresis is dependent on renal function, dietary proteins and hydration level ${ }^{17}$. In our group of healthy volunteers the hydration level probably has the largest influence on diuresis, but other factors can be an explanation for the large variation between individuals. This may also have an impact in patients with for example overactive bladder complaints, as they often reduce fluid intake.

Two patterns of "focused introspection" curves were found (figure 2). Within an individual, the shape of the curve and the volume/intensity points showed a good agreement over the three sessions with ten days interval (figure 3). Although an individual may have a specific "focused introspection" curve, a transition from convex to S-shape might be possible if the individual is stimulated to postpone longer. The best agreement was found between session 2 and 3 . This suggests a "learning" effect meaning that the participants learned to evaluate their bladder sensations consciously. This effect was most important for the volume at first sensation, which appears the most variable. This is in accordance with the studies on bladder sensation during cystometry, during which first sensation of filling also appears variable and due to level of focus or attention towards bladder filling $^{18}$. Phase 3 , which represents the phase during which bladder sensation rapidly increases, appears the most stable over the different sessions. The calculated slope of this phase, represents the velocity of sensation intensity change and is a quantitative parameter to indicate sensation change. The slope is different for healthy volunteers and $\mathrm{OAB}$ patients (Heeringa et al, in press). Similar sensation curves have been described during cystometric bladder filling ${ }^{19}$. Eighteen of the twenty-five curves described in that study were linear, whereas only 7 were "non-linear” and resemble the curves from the present study. Considering the animal afferent recording data that show that at physiological filling rate, a variety in afferent response pattern exists that disappears if the filling rate is increased to supraphysiological rates ${ }^{16}$, it is easy to conceive that the "linear" curve at $50 \mathrm{ml} / \mathrm{min}^{19}$ might be due to high filling rate. This again reinforces the need to use methods that are as physiologically as possible. 
'Focused introspection' during a naturally increased diuresis: description and repeatability of a new method to study bladder sensation non-invasively

\section{Conclusion}

A strict water loading protocol can induce a constant high diuresis that gives the opportunity to evaluate bladder sensations during a non-invasive bladder filling. The absolute need to void may develop in two different patterns, but for a given person, the pattern did not change over the three independent sessions with ten days intervals.

\section{References}

1. Motzkin, D.: The significance of deficient bladder sensation. J Urol, 100: 445, 1968

2. Van Meel, T. D., Wyndaele, J. J.: Reproducibility of urodynamic filling sensation at weekly interval in healthy volunteers and in women with detrusor overactivity. Neurourol Urodyn, Apr 28. doi: 10.1002/nau.21100., 2011

3. Erdem, E., Akbay, E., Doruk, E. et al.: How reliable are bladder perceptions during cystometry? Neurourol Urodyn, 23: 306, 2004

4. Erdem, E., Tunckiran, A., Acar, D. et al.: Is catheter cause of subjectivity in sensations perceived during filling cystometry? Urology, 66: 1000, 2005

5. De Wachter, S., Van Meel, T. D., Wyndaele, J. J.: Can a faked cystometry deceive patients in their perception of filling sensations? A study on the reliability of spontaneously reported cystometric filling sensations in patients with non-neurogenic lower urinary tract dysfunction. Neurourol Urodyn, 27: 395, 2008

6. Sorensen, S. S., Nielsen, J. B., Norgaard, J. P. et al.: Changes in bladder volumes with repetition of water cystometry. Urol Res, 12: 205, 1984

7. De Wachter, S., Wyndaele, J. J.: Frequency-volume charts: a tool to evaluate bladder sensation. Neurourol Urodyn, 22: 638, 2003

8. Naoemova, I., De Wachter, S., Wyndaele, J. J.: Comparison of sensation-related voiding patterns between continent and incontinent women: a study with a 3-day sensation-related bladder diary (SR-BD). Neurourol Urodyn, 27: 511, 2008

9. Cardozo, L., Hessdorfer, E., Milani, R. et al.: Solifenacin in the treatment of urgency and other symptoms of overactive bladder: results from a randomized, double-blind, placebo-controlled, rising-dose trial. BJU Int, 102: 1120, 2008

10. Dmochowski, R. R., Peters, K. M., Morrow, J. D. et al.: Randomized, double-blind, placebo-controlled trial of flexible-dose fesoterodine in subjects with overactive bladder. Urology, 75: 62, 2010

11. De Wachter, S., Wyndaele, J. J.: Can the sensory threshold toward electrical stimulation be used to quantify the subjective perception of bladder filling? A study in young healthy volunteers. Urology, 57: 655, 2001

12. Chapple, C. R., Artibani, W., Cardozo, L. D. et al.: The role of urinary urgency and its measurement in the overactive bladder symptom syndrome: current concepts and future prospects. BJU Int, 95: 335, 2005

13. Wyndaele, J. J.: The normal pattern of perception of bladder filling during cystometry studied in 38 young healthy volunteers. J Urol, 160: 479, 1998

14. Abrams, P., Cardozo, L., Fall, M. et al.: The standardisation of terminology of lower urinary tract function: report from the Standardisation Sub-committee of the International Continence Society. Neurourol Urodyn, 21: 167,2002

15. Robertson, A. S.: Behaviour of the human bladder during natural filling: the Newcastle experience of ambulatory monitoring and conventional artificial filling cystometry. Scand J Urol Nephrol Suppl, 201: 19, 1999

16. De Wachter, S., De Laet, K., Wyndaele, J. J.: Does the cystometric filling rate affect the afferent bladder response pattern? A study on single fibre pelvic nerve afferents in the rat urinary bladder. Neurourol Urodyn, 25: 162, 2006

17. de Rouffignac, C.: Effects of water balance, diet and antidiuretic-hormone administration on the renal excretion of water. Scand J Urol Nephrol Suppl, 202: 31, 1999

18. Wyndaele, J. J., De Wachter, S.: Cystometrical sensory data from a normal population: comparison of two groups of young healthy volunteers examined with 5 years interval. Eur Urol, 42: 34, 2002

19. Dompeyre, P., Fritel, X., Bader, G. et al.: Bladder sensitivity testing using a visual analogue scale: comparative cystometric study on women. Neurourol Urodyn, 26: 350, 2007 



\section{Normal bladder sensations in healthy volunteers: a focus group investigation.}

Rhea Heeringa ${ }^{1,2}$, Stefan G.G. de Wachter ${ }^{1,2}$, Philip E.V. van Kerrebroeck ${ }^{1,2}$, Gommert A. van Koeveringe ${ }^{1,2}$

1. Department of Urology

Maastricht University Medical Centre; The Netherlands

2. European Graduate School of Neuroscience (EURON)

Maastricht University Medical Centre; The Netherlands

Neurourology and Urodynamics - 30 (7) : 1350-5, 2011

Epub: 2011 May 23 


\section{Abstract}

Aims: to describe the bladder sensations experienced during non-invasive rapid bladder filling in a controlled setting in healthy volunteers.

Methods: three groups of healthy volunteers, total 11 subjects ( 4 men, 7 women), participated in three consecutive focus group sessions. Before each session a strict water loading protocol was given. The first two sessions focused on how participants described and experienced bladder sensations in daily life and during a physiological bladder filling with constant focus on their bladder. The third session focused on verifying the interpretation of the data gathered so far and describing the pattern of sensations.

Results: during the focus group sessions with constant focus on their bladder, all participants experienced their bladder sensation as a continuous progression. Healthy subjects describe their bladder sensation(s) as a 'pressure' and a 'tingling' sensation, but a terminology ranging from no sensations to an absolute need to void is used by all participants and better describes the pattern in which the need to void develops in healthy volunteers.

Conclusions: by means of focus group research it is possible to describe the development of normal bladder sensation and to refine terminology used by healthy participants. The findings show that two types of bladder sensations are reported spontaneously by healthy uninitiated volunteers, pressure and a tingling sensation, of which the first develops in a continuous manner. The pattern in which the absolute need develops is described by the terms: no sensation, weak awareness, stronger awareness, weak need, stronger need and absolute need to void. 


\section{Introduction}

Our knowledge of bladder sensations is largely derived from urodynamic studies. Using this method Denny-Brown et al. ${ }^{1}$ and Nathan ${ }^{2}$ were the first to describe bladder sensations. Their studies described vague abdominal sensations when the bladder fills, growing sensations associated with a general desire to void and strong sensations associated with imminent micturition. Sensations of awareness and desire were located in the bladder, while sensations of imminent micturition would originate from lower down, possibly the proximal urethra ${ }^{1,2}$.

Years later bladder sensations were systematically studied in healthy volunteers during cystometry ${ }^{3,4}$. It was accepted that bladder filling sensations consisted of three different sensations, which could be described as a first sensation of filling, a first desire to void and a strong desire to void. These terms have been endorsed by the International Continence Society (ICS) ${ }^{5}$ and are now regarded as the accepted terms to describe the normal pattern of bladder filling. However, from these studies it is unclear how these terms and descriptions have arisen: were these descriptions provided by the volunteers themselves, or merely derived form the observers' interpretation?

To our knowledge, no study has addressed how individuals describe normal bladder sensation and how bladder sensation develops during a non-invasive bladder filling. This study is the first to report how healthy volunteers describe their perceived sensation(s) during non-invasive bladder filling in their own terms and how the pattern of a full bladder develops. This was evaluated by means of interactive focus group sessions that addressed bladder sensation in daily life and during constant focus on the bladder.

\section{Material and Methods}

In accordance to the guidelines ${ }^{6}$ we organised focus group discussions with healthy volunteers. All subjects (4 male; 7 female) had no urological symptoms and an uneventful history regarding abdominal, gynaecological or urological surgery. The focus group design is an iterative process of individuals that discuss on a specific subject. Questions that were addressed were: how does bladder sensation develop?, which words are used to describe bladder sensation?, and where are sensations located? The discussions are repeated in different groups until, in a new group, no new information is obtained. Maximally four participants were allowed per group to ensure input from all participants and a thorough discussion. The discussions were held in Dutch. For comparative purposes in this article the Dutch and corresponding English words are listed in table 1. All sessions were moderated by the first author.

\section{Focus group session}

Two sessions ( \pm 120 minutes each) were conducted with each group. During the first session questions as described above were asked, while in the second session the results of the first session were discussed and if necessary information was added or modified. A controlled non-invasive bladder filling by constant high diuresis was aimed at by applying a water loading protocol. One hour before the start of the sessions participants were asked to drink $1000 \mathrm{ml}$ of water. During the sessions they were asked to 
drink another $200 \mathrm{ml}$ every 10 minutes, with a maximum of an extra $1000 \mathrm{ml}$. This water loading protocol was chosen to reach maximum bladder capacity by natural diuresis over a period of 1 to 2 hours (based on preliminary work). To start with an empty bladder, participants voided at the beginning of the session.

To avoid any possible observer bias the moderator avoided the use of any terminology that could describe the characteristics of sensations. The group was asked to discuss the development of bladder sensation, the words to describe bladder sensation and the location of bladder sensation. By interactive discussion between participants an overall group consensus was aimed for. Besides the discussion participants were asked to fill out two different forms. The first form was an empty $X Y$-graph with an open-end time scale on the $X$-axis and intensity of bladder fullness sensation starting from 0 (empty bladder) to $100 \%$ (absolute need) on the Y-axis. It was used to study the development of bladder sensation. The characteristic and location of the sensation, were described on the second form in their own words. They were asked to fill in both forms every ten minutes and also whenever they noticed any change. The protocol ended with an absolute need to void and voided volumes were measured.

During the first session every group of participants had the opportunity to describe the experienced sensations in their own words, without any prior information given. During the second session with group 2 and 3 , the results from the sessions with the previous group were discussed and compared with their own experiences according to the guidelines on focus groups ${ }^{6}$. If necessary information was adapted. New groups were formed until no new information was obtained in subsequent groups.

\section{Validation session}

At the end of the protocol sessions an additional "validation" session was organised, with all previously consulted participants. An overall summary and interpretation of the data was given and participants were asked to comment on this. Again the water loading protocol was used and participants graded their bladder sensation on an empty graph. A new form contained the "consensus" words which are based on the information gathered in the protocol sessions. The words describe the pattern in which bladder sensations develop. The "consensus" words were: no sensation, initial awareness, stronger awareness, initial desire, stronger desire and absolute need to void. Both forms were filled in every 10 minutes and whenever anything changed.

\section{Statistical analyses}

All $p$ values given in this article are calculated by Wilcoxon signed rank test with Bonferroni correction.

\section{Results}

Three groups (of in total 4 men and 7 women; mean age 24.4 years) were required to obtain "non changing" information. Both the duration of a session (mean $62 \pm 12 \mathrm{~min}$ ) and the rate of diuresis during a session (mean $12.1 \pm 3.4 \mathrm{ml} / \mathrm{min}$ ) were not different throughout all sessions (respectively $p>0.17$ and $p>0.12$ ). 


\section{Terminology - participants}

Participants found it hard to describe their bladder sensations. Care was taken to stimulate them to describe sensation in their own nomenclature and only vague possibilities, like pleasant or painful, were given as examples. Different descriptions were used but after thorough discussions between participants all terms were brought back to two denominators: pressure and tingling (figure 1 and table 1). Five participants only experienced a pressure during the filling phase whilst the other participants could also describe a tingling sensation (figure 2).

Six participants had no sensation post-void, whereas one female and one male participant experienced a tingling feeling in the genital area. The other 3 participants experienced a sensation post-void that was not intense enough to be described. The volume at which a first sensation was noted as well as its intensity were not statistically different between all sessions (respectively all $p>0.06$ and all $p>0.22$ ). In daily life, the sensation(s), mainly in the beginning of the filling phase, can be (actively) displaced from the mind temporarily. However when asked, the sensations can be addressed at any time. Men and women described the sensations similarly.

The absolute need to void was experienced as a sensation of strong pressure from the bladder pointing to the genital area as well as to the abdomen with or without a tingling sensation in the genital area. This sensation made participants ask whether they were allowed to void. The mean voided volume was $745.9 \pm 223.1 \mathrm{ml}$ (range: $240.0-1120.0$ $\mathrm{ml})$ and was not statistically different between the three sessions $(p=1.00)$.

Figure 1 shows the process of the development of the accepted terms that describe the characteristic or the pattern of normal bladder sensations. Participants spontaneously came up with two groups of initial participants terms which could be further brought back to the denominators pressure and tingling.

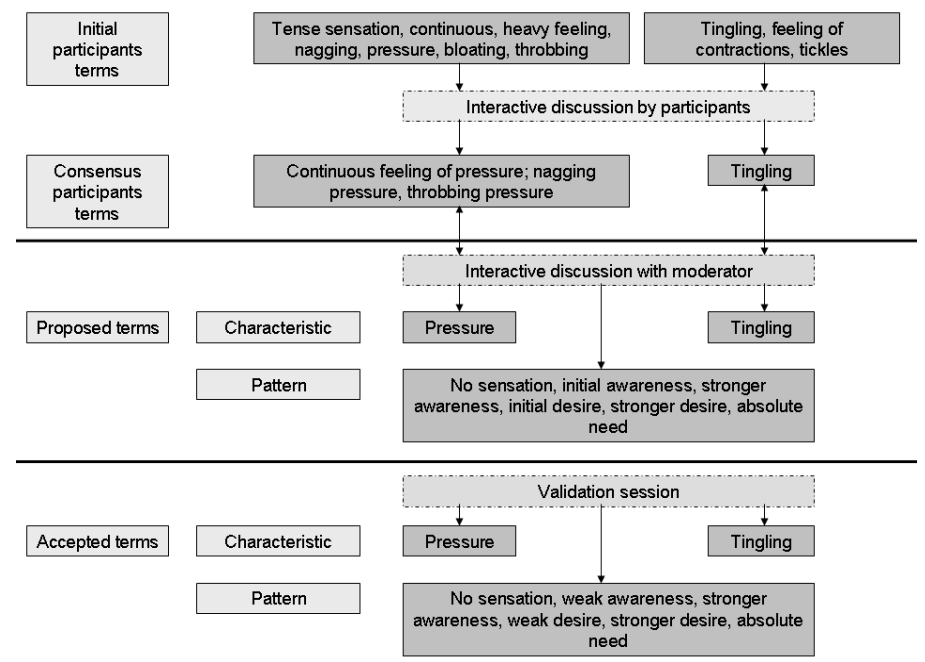




\section{Chapter 5}

Figure 2 shows examples of the relationship between the type of sensation and the bladder volume for four different participants in one session. It shows that the sensation of pressure only increases in intensity and is continuous. The "tingling" sensation however, may also decrease in intensity during one filling session and is not per se continuous.
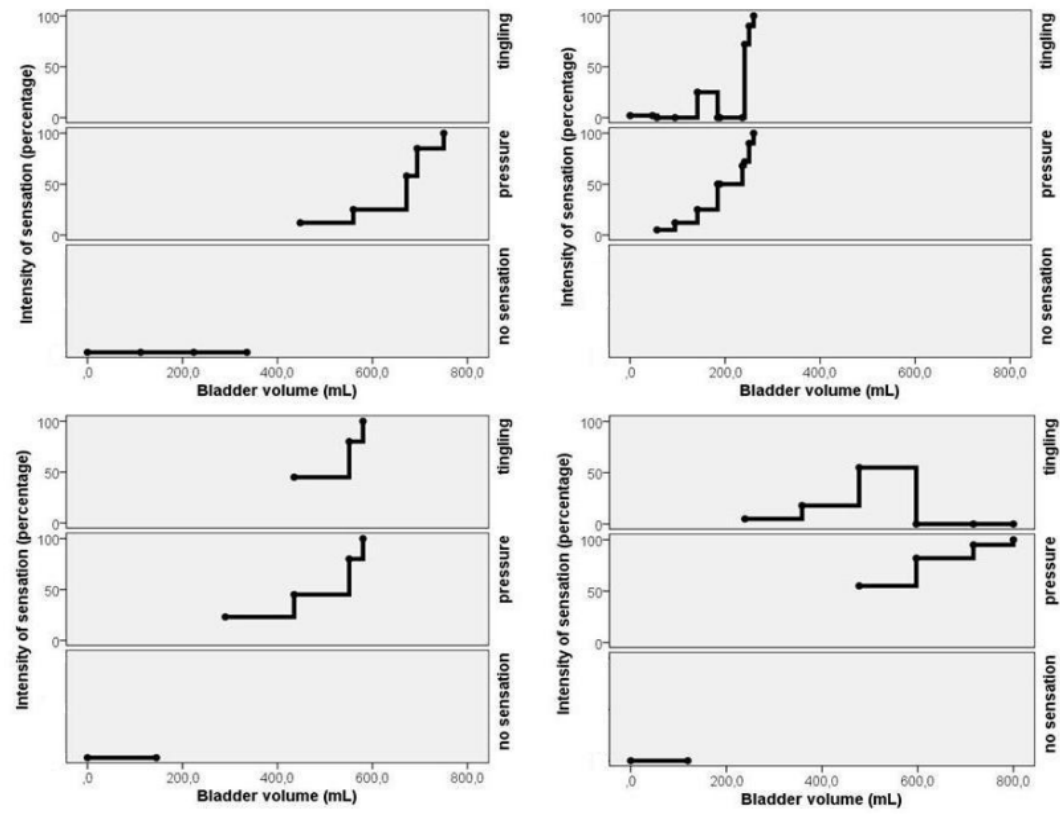

Table 1 shows all used terminology in Dutch and in English. A native English speaker has been closely involved in translating the terminology from Dutch into English.

\begin{tabular}{|l|l|}
\hline Terminology in Dutch & Terminology in English \\
\hline Gespannen & Tense \\
\hline Zwaar & Heavy \\
\hline Zeurend & Nagging \\
\hline Druk & Pressure \\
\hline Opgeblazen & Bloating \\
\hline Kloppend & Throbbing \\
\hline Tintelend & Tingling \\
\hline Gevoel van samentrekkingen & Feeling of contractions \\
\hline Prikkels & Tickles \\
\hline Onaangenaam & Unpleasant \\
\hline Pijnlijk & Painful \\
\hline Geen sensatie & No sensation \\
\hline Zwakke gewaarwording & Weak awareness \\
\hline Sterkere gewaarwording & Stronger awareness \\
\hline Zwakke aandrang & Weak desire \\
\hline Sterkere aandrang & Stronger desire \\
\hline Moeten plassen & Absolute need \\
\hline
\end{tabular}




\section{Terminology - validation session}

To verify our interpretation, the participants received "consensus terminology" based on information gathered from previous sessions (figure 1). Though all participants agreed to this terminology they changed the word "initial" into "weak" because "initial" meant that the sensation could only be experienced once, but there were cases in which the sensation did not change from initial to stronger awareness in one time-point. The word "weak" has previously been used to describe an early bladder sensation ${ }^{7}$. Detailed information for each sensation can be found in table 2 . Though clearly experienced during the session, the weak and growing awareness are, in daily life, not always intense enough to be noticed spontaneously. It may depend on the activity and the level of focus a subject has at that moment. The strong desire to void as well as the absolute need to void could also be experienced as painful or unpleasant.

Table 2 shows a description of the terminology during session 3 even as its duration, volume and intensity values.

\begin{tabular}{|c|c|c|c|c|}
\hline Sensation & Description & $\begin{array}{l}\text { Mean } \\
\text { duration } \\
\text { (min); (SD) }\end{array}$ & $\begin{array}{l}\text { Mean } \\
\text { volume } \\
(\mathrm{ml}) ;(\mathrm{SD})\end{array}$ & $\begin{array}{l}\text { Mean } \\
\text { intensity } \\
\text { (\%); (SD) }\end{array}$ \\
\hline No sensation & $\begin{array}{l}\text { No sensation in the lower abdomen } \\
\text { or genitalia }\end{array}$ & $\begin{array}{l}23.5 \\
(8.9)\end{array}$ & $\begin{array}{l}105.9 \\
(106.8)\end{array}$ & $\begin{array}{l}0.1 \\
(0.4)\end{array}$ \\
\hline Weak awareness & $\begin{array}{l}\text { Weak awareness of the bladder. } \\
\text { Sensation in the background. Not } \\
\text { spontaneously noticed. Either pres- } \\
\text { sure or tingling sensation. }\end{array}$ & $\begin{array}{l}10.1 \\
(1.8)\end{array}$ & $\begin{array}{l}254.6 \\
(149.6)\end{array}$ & $\begin{array}{l}8.9 \\
(4.2)\end{array}$ \\
\hline Stronger awareness & $\begin{array}{l}\text { Stronger awareness of the bladder } \\
\text { but no desire to void. Either pressure } \\
\text { and/or tingling sensation. }\end{array}$ & $\begin{array}{l}12.8 \\
(7.6)\end{array}$ & $\begin{array}{l}364.7 \\
(154.2)\end{array}$ & $\begin{array}{l}23.9 \\
(9.0)\end{array}$ \\
\hline Weak desire & $\begin{array}{l}\text { Weak desire to void. If I would be } \\
\text { doing nothing I would go to the toilet. } \\
\text { Either pressure and/or tingling } \\
\text { sensation. }\end{array}$ & $\begin{array}{l}8.1 \\
(3.2)\end{array}$ & $\begin{array}{l}522.9 \\
(165.7)\end{array}$ & $\begin{array}{l}50.8 \\
(11.4)\end{array}$ \\
\hline Stronger desire & $\begin{array}{l}\text { Stronger desire to void. I search a } \\
\text { toilet actively. Either pressure and/or } \\
\text { tingling sensation. }\end{array}$ & $\begin{array}{l}7.9 \\
(4.1)\end{array}$ & $\begin{array}{l}539.2 \\
(214.8)\end{array}$ & $\begin{array}{l}78.7 \\
(14.3)\end{array}$ \\
\hline Absolute need & $\begin{array}{l}\text { Can be unpleasant or painful. Pres- } \\
\text { sure with or without a tingling sensa- } \\
\text { tion. }\end{array}$ & $\begin{array}{l}1.1 \\
(2.1)\end{array}$ & $\begin{array}{l}745.0 \\
(180.4)\end{array}$ & $\begin{array}{l}96.7 \\
(7.3)\end{array}$ \\
\hline
\end{tabular}




\section{Location}

In both sexes, the tingling sensation is always felt in the genitalia while the sensation of pressure is felt either in the lower abdomen or in the genitalia. Figure 3 shows that there is not a sole pattern where bladder sensations are felt.

Participants that only experience a pressure, have their first sensation in the lower abdomen which will expand to the genitalia. Participants, also experiencing a tingling sensation, are more likely to feel their first sensation in the genitalia, sometimes combined with a sensation in the lower abdomen. In these cases there is no clear pattern in which the sensation will develop per location over time. The pattern can even change between sessions in one individual.

Figure 3 shows the relationship between the location of the sensation and the bladder volume for four participants during session 1.
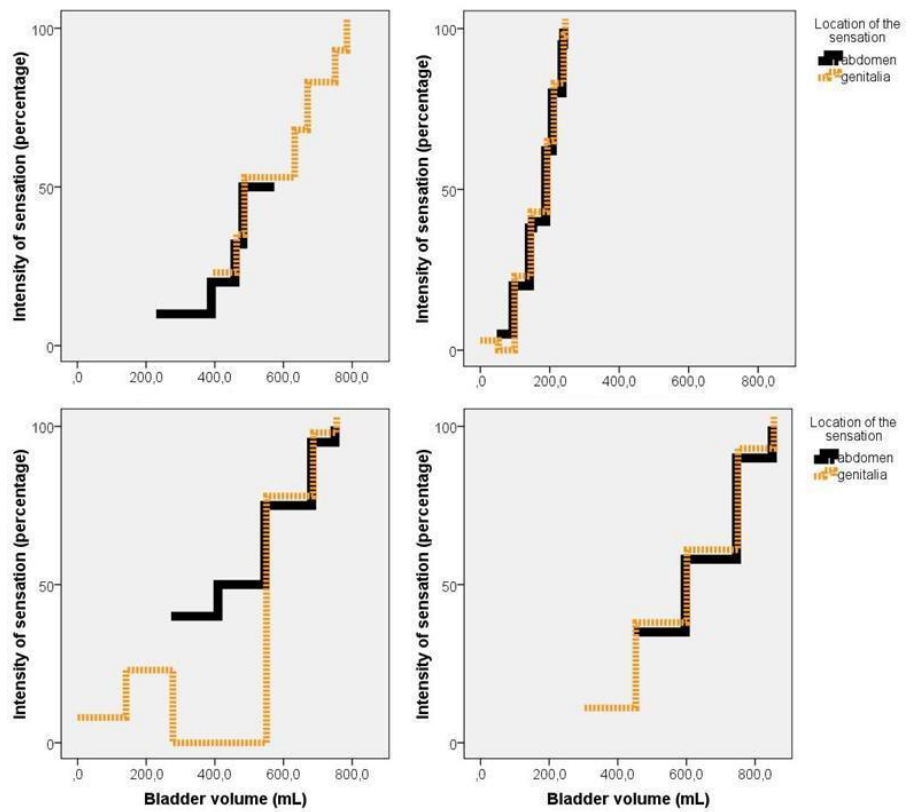

\section{Discussion}

A clear insight into normal bladder sensation is essential in order to understand pathological states of the lower urinary tract. Moreover, we need a clear, accepted standardised terminology to describe normal bladder sensations because the current terminology is interpreted in different ways ${ }^{8,9}$. This is easier said than done because sensations are subjective and therefore hard to study. In the field of psychology, these kind of issues are specifically studied using focus group research. In order to describe normal bladder sensation(s) we organised interactive focus group sessions with volunteers.

Focus groups are a qualitative research method used to explore thoughts from subjects. Behaviours and motivation can be discussed in depth and interpretation of the infor- 
mation can be verified ${ }^{6}$. To evaluate how volunteers describe bladder sensations, we therefore found this method very useful. Experiences, motivation and words from different participants could be discussed and interpretation of the data could be verified directly. Moreover, by asking only open-end questions and on forehand using no terminology of our own, the participants gave us an unbiased description of their sensations.

Before this study, terms to describe bladder sensations, are derived from cystometry studies. Sensations were studied in healthy volunteers ${ }^{3}$ and three distinct sensations were described. This first sensation (i) in the lower pelvis, described as weak and fluctuating, is probably only perceived during artificial filling. The first desire to void (ii) is described as a constant sensation gradually becoming more intense. The strong desire to void (iii) is a constant sensation which will lead to actively finding a place to void. Finally the sensation of urge or great tension would develop ${ }^{3}$. Years later this study was repeated in another 50 volunteers and the same results were found ${ }^{4}$. The above terminology describes a pattern in which the need to void will develop and is widely used in practice. However, because the characteristic of the different sensations is unknown we cannot be sure whether these are in fact three separate sensations or whether it is the same sensation building up in intensity. Furthermore we do not know if these sensations are also experienced during physiological bladder filling.

Using sensation-related bladder diaries (SR-BD) we can study bladder sensations noninvasively ${ }^{10,11}$. Unfortunately, the SR-BD only gives insight into the bladder sensation just before voiding and not into the way in which the need to void has developed. Moreover, the SR-BD uses predefined terms to which subjects need to relate their own sensation.

Another issue with previous studies is that the available data on sensations might be biased through the instruction given to the patient or the interpretation of the data by the researcher. For example two studies showed that even when the bladder is not filled during cystometry, many participants still say to experience a first sensation of filling, a first and strong desire ${ }^{12,13}$. However, this protocol was repeated by another group that showed that only 3 out of 59 patients experienced bladder sensations without the bladder being filled ${ }^{14}$. This suggests that an experimenter has a major impact on the results of a study. Dompeyre et al. showed that the method used to evaluate bladder sensation may influence the pattern in which bladder sensation is described ${ }^{15}$. It can be argued that the application of a water loading protocol induces a diuresis different from daily life and therefore not physiological. However, the natural non-invasive filling in a high diuresis protocol is likely to be more physiological than a recording of sensations during an invasive urodynamic investigation. Moreover, to our knowledge this is the first study that describes normal bladder sensations in a controlled focused setting without biasing the participants with our terminology.

In their own words, healthy subjects describe the characteristic of their bladder sensations as a pressure and a tingling sensation, though the latter is not experienced by everyone. Pressure is the main sensation which will gradually build up in intensity, trigger voiding and is continuous. It is felt in the lower abdomen, genitalia or at both locations at the same time. The term pressure has been used previously to describe a continuous bladder sensation ${ }^{7}$. Some participants experience a secondary tingling sensation that can or cannot be felt at the same time as the pressure. This tingling sensation has no 
continuous pattern and is felt mainly at the beginning or the end of the bladder filling. The pressure and tingling sensations are not related to the pattern of awareness, desire and need, as described below, but purely describe the characteristic of normal bladder sensations.

In order to differentiate better between several phases of bladder filling we introduced a more specific consensus terminology during the validation session. This terminology came about through interpreting the discussions of the focus group experiment sessions. Using this terminology we can describe the normal pattern of bladder sensations in a standardized way. The consensus terminology looks like the currently accepted pattern of first sensation, first desire and strong desire but there are some important differences. First, the consensus terminology was based on words used by the volunteers and afterwards verified by all previously consulted participants. It is unknown whether this is the case with the now accepted way to describe the pattern of normal bladder sensations. Secondly, according to the participants it is important to leave out the term "first" because subjects cannot relate to this term as a description of a bladder sensation. It is better replaced by "weak" to which all volunteers can relate. Thirdly, the word "filling" in "first sensation of filling" could better be replaced by "awareness". The word "filling" is not spontaneously used but participants mention to be aware of a sensation that is different from a desire to void. Though using different terms might indicate that there are several episodic sensations, as is now accepted ${ }^{16}$, this is not the case. The terms purely describe different phases in bladder filling without implicating different types of sensations.

Several afferent pathways are responsible for communicating sensations arising from the lower urinary tract to other areas. It is believed that each afferent pathway is responsible for a specific bladder sensation, for example bladder fullness or desire to micturate ${ }^{17}$. However, our study shows that pressure can be felt during several phases of bladder filling and in areas innervated by more than one afferent pathway. Therefore it appears better to state that different afferent pathways communicate sensations from different locations without implicating differences in characteristics or place in the pattern of bladder filling. Furthermore, since sensations appear to be continuous and perceived at different locations at the same time, this would suggest involvement of different afferents or nerves, which can easily be reconciled with the 'afferent noise' hypotheses ${ }^{18}$.

The study has the limitation that we used young volunteers. At higher ages however, a volunteer group is more likely to have subclinical disorders related to the bladder filling and voiding. Therefore these young participants, not complaining about urological problems, are more likely to be healthy individuals. Moreover, all had an uneventful history regarding abdominal, gynaecological or urological surgery. All participants are highly educated and we are aware that people with other educational levels might describe their bladder sensations differently. But because the focus group approach is the first study of its kind in the evaluation of bladder sensation terminology, we have chosen a population that was expected to be capable of accurately describing their sensations. All participants mentioned that compared to daily life, they felt sensations more rapidly during the session, because of the intense focus on their bladder. However other factors such as posture, activity level, bowel status, drinking behaviour and hydration status might also influence how bladder sensations are perceived, but this was not specifically addressed in this study. 
Furthermore this is the first study that shows how normal bladder sensations are experienced and described by healthy volunteers without biasing them with our terminology. We would suggest that the next step would be to repeat this study in a larger group of native English volunteers and patients with lower urinary tract symptoms.

\section{Conclusions}

By means of focus group research and constant focus on the bladder, it is possible to describe normal bladder sensations and to refine the terminology used by healthy participants. By having clear insight into the characteristics and pattern of normal sensations we can start to unravel which- and where changes occur in pathological states.

This is the first study that shows how normal bladder sensations are experienced and described. The characteristics of the normal bladder sensations are a pressure or a tingling sensation. The pattern in which the need to void develops can be described more specifically by using the terms: no sensation, weak awareness, stronger awareness, weak need, stronger need and absolute need to void.

\section{References}

1. Denny-Brown, D., Robertson, E. G.: On the physiology of micturition. Brain, 56: 149, 1933

2. Nathan, P. W.: Sensations associated with micturition. Br J Urol, 28: 126, 1956

3. Wyndaele, J. J.: The normal pattern of perception of bladder filling during cystometry studied in 38 young healthy volunteers. J Urol, 160: 479, 1998

4. Wyndaele, J. J., De Wachter, S.: Cystometrical sensory data from a normal population: comparison of two groups of young healthy volunteers examined with 5 years interval. Eur Urol, 42: 34, 2002

5. Abrams, P., Cardozo, L., Fall, M. et al.: The standardisation of terminology of lower urinary tract function: report from the Standardisation Sub-committee of the International Continence Society. Am J Obstet Gynecol, 187: 116, 2002

6. Morgan, D. L.: The Focus Group Guidebook. London: Sage Publications, p. 103, 1998

7. Digesu, G. A., Basra, R., Khullar, V. et al.: Bladder sensations during filling cystometry are different according to urodynamic diagnosis. Neurourol Urodyn, 28: 191, 2009

8. Blaivas, J. G., Panagopoulos, G., Weiss, J. P. et al.: Two types of urgency. Neurourol Urodyn, 28: 188, 2009

9. Digesu, G. A., Khullar, V., Panayi, D. et al.: Should we explain lower urinary tract symptoms to patients? Neurourol Urodyn, 27: 368, 2008

10. De Wachter, S., Wyndaele, J. J.: Frequency-volume charts: a tool to evaluate bladder sensation. Neurourol Urodyn, 22: 638, 2003

11. Naoemova, I., De Wachter, S., Wyndaele, J. J.: Comparison of sensation-related voiding patterns between continent and incontinent women: a study with a 3-day sensation-related bladder diary (SR-BD). Neurourol Urodyn, 27: 511, 2008

12. Erdem, E., Akbay, E., Doruk, E. et al.: How reliable are bladder perceptions during cystometry? Neurourol Urodyn, 23: 306, 2004

13. Erdem, E., Tunckiran, A., Acar, D. et al.: Is catheter cause of subjectivity in sensations perceived during filling cystometry? Urology, 66: 1000, 2005

14. De Wachter, S., Van Meel, T. D., Wyndaele, J. J.: Can a faked cystometry deceive patients in their perception of filling sensations? A study on the reliability of spontaneously reported cystometric filling sensations in patients with non-neurogenic lower urinary tract dysfunction. Neurourol Urodyn, 27: 395, 2008

15. Dompeyre, P., Fritel, X., Bader, G. et al.: Bladder sensitivity testing using a visual analogue scale: comparative cystometric study on women. Neurourol Urodyn, 26: 350, 2007

16. Chapple, C. R., Artibani, W., Cardozo, L. D. et al.: The role of urinary urgency and its measurement in the overactive bladder symptom syndrome: current concepts and future prospects. BJU Int, 95: 335, 2005

17. Torrens, M., Morrison, J. F. B.: The Physiology of the Lower Urinary Tract. Berlin Heidelberg: Springer-Verlag, pp. 89-94, 1987

18. Gillespie, J. I., van Koeveringe, G. A., de Wachter, S. G. et al.: On the origins of the sensory output from the bladder: the concept of afferent noise. BJU Int, 103: 1324, 2009 



\section{Do patients with OAB experience bladder sensations in the same way as healthy volunteers? A focus group investigation.}

R. Heeringa ${ }^{1}$, G. A. van Koeveringe ${ }^{1,2}$, B. Winkens ${ }^{3}$, P. E. V. A. van Kerrebroeck $^{1,2}$, S. G. G. de Wachter ${ }^{1,2}$

1. Department of Urology

Maastricht University Medical Centre; The Netherlands

2. European Graduate School of Neuroscience (EURON)

Maastricht University Medical Centre; The Netherlands

3. University of Maastricht

Department of Methodology and Statistics

Maastricht University Medical Centre; The Netherlands

Neurourology and Urodynamics - 31 (4) : 521-5, 2012

Epub: 2012 March 6 


\section{Abstract}

Aims: To describe the terminology and pattern of bladder sensations experienced during non-invasive rapid bladder filling in a controlled setting in patients with $O A B$ and to compare these results with a previous study conducted in healthy volunteers.

Methods: Three groups of patients with $O A B$, in total 10 patients, participated in three consecutive focus group sessions. Before each session a strict water loading protocol was given. During the first two sessions, participants described how they experienced their bladder sensations in daily life and during a non-invasive bladder filling with constant focus on their bladder. The third session focused on verifying the interpretation of the data gathered and describing the pattern of sensations.

Results: Patients describe their bladder sensations as a pressure or a tingling sensation and the pattern can be described by terms ranging from no sensation to an absolute need to void. The absolute need to void may develop suddenly or more slowly progressive. The mean development of bladder sensation is significantly different between patients and healthy volunteers as well as their average diuresis.

Conclusions: Patients with $\mathrm{OAB}$ describe their bladder sensations as a pressure or a tingling sensation. There appear to be two types of urgency: a sudden absolute need to void and a slowly developing absolute need to void. Furthermore bladder sensation develops significantly different in volunteers than in OAB patients. 


\section{Introduction}

Urodynamic studies are used to evaluate the development of bladder sensations ${ }^{1}$. Unfortunately this technique is invasive and fills the bladder retrograde. Recently interactive focus group sessions have been described to study bladder sensations non-invasively ${ }^{2}$. This qualitative research method can be used to explore thoughts from subjects ${ }^{3}$ and was found to be useful in studying bladder sensations in healthy volunteers and helpful to refine the terminology that was used to describe these sensations. In order to induce a constant forced diuresis a water loading protocol was used ${ }^{4}$, and during bladder filling healthy subjects described the development and characteristics of bladder sensation as either a pressure or tingling sensation ${ }^{2}$.

The purpose of the current study was to repeat the focus group sessions using the water loading protocol in patients with overactive bladder symptoms, and to study the terms used to describe bladder sensation and development of bladder sensation during a noninvasive bladder filling. These data will be compared with the terminology and pattern described by healthy volunteers previously ${ }^{2}$.

\section{Material and Methods}

In accordance to the guidelines ${ }^{3}$ focus group discussions with patients with overactive bladder symptoms $(O A B)$ were organised. Patients were selected from the outpatient clinic and if on anticholingergic treatment, this was stopped three weeks prior to the sessions. The focus group design is an iterative process of individuals that discuss a specific topic. The discussions are repeated in different groups until, in a new group, no new information is obtained. Maximally five participants were allowed per group to ensure maximum input from all participants and a thorough discussion. All sessions were moderated by the first author $(\mathrm{RH})$. The current protocol is identical to the focus group protocol conducted in healthy volunteers ( 4 men, 7 women, mean age 24.4 years) previously $^{2}$. The language used during the discussions was Dutch.

\section{Focus group sessions}

Within each focus group, two sessions ( \pm 120 minutes each) were conducted. A noninvasive bladder filling thorough constant high diuresis was aimed at by applying a water loading protocol, as previously described ${ }^{2}$. Participants were asked to drink 1 litre during the hour before the session and another $200 \mathrm{ml}$ each 10 minutes during the session. To start with an empty bladder, participants voided at the beginning of the session. To avoid observer bias, the moderator avoided the use of any terminology that could describe characteristics of sensations.

The group was asked to discuss the development of bladder sensations, the words to describe bladder sensations and the location of bladder sensations. During the session the participants were asked to fill out two different forms. The first form was an empty $\mathrm{XY}$-graph with an open-end time scale on the X-axis and intensity of bladder fullness sensation starting from 0 (empty bladder) to $100 \%$ (absolute need) on the Y-axis. It was used to study the development of bladder sensations. The second form was used to describe the characteristic and location of the sensations in their own words. They were 
asked to fill out both forms every ten minutes and also whenever they noticed any change in bladder sensations. The protocol ended with an absolute need to void and voided volumes were measured. Finally by interactive discussion between participants an overall group consensus on terminology was aimed for.

During the first session every group of participants had the opportunity to describe the experienced sensations in their own words, without any prior information given. During the second session with group 2 and 3 , the results from the sessions with the previous group were discussed and compared with their own experiences according to the guidelines on focus groups ${ }^{3}$. If necessary, information was adapted. New groups were formed until no new information was obtained in subsequent groups.

\section{Additional validation session}

At the end of the protocol sessions an additional "validation" session was organised, which included all previously consulted participants. An overall summary and interpretation of the data was given and participants were asked to comment on this. Again the water loading protocol was followed and participants graded their bladder sensation on an empty graph. A new form contained the "consensus" words which are based on the focus group sessions with healthy volunteers ${ }^{2}$. The words describe the pattern in which bladder sensations develop. The consensus words were: no sensation, weak awareness, stronger awareness, weak desire, stronger desire and absolute need to void. Both forms were filled out every 10 minutes and whenever anything changed.

\section{Statistical analyses}

The difference in continuous variables, like duration of the session, diuresis, volume and intensity at first sensation, and voided volume, between sessions are compared using Wilcoxon signed rank test with Bonferroni correction. Differences between patients and volunteers were tested with Mann-Whitney U-test. To adjust for correlated measurements within individuals, linear mixed models with a random intercept were used to evaluate the effect of study group on introspection curves and on bladder volume. A p-value $\leq 0.05$ was considered as statistically significant. All analyses were performed using SPSS $® 18$.

\section{Results}

Three groups of patients, within each group at least 3 participants, were required until no new information was obtained from the focus group sessions. In total 5 female and 5 male patients (mean age 57.2 years) were studied. Unless it is specifically indicated, the results that are presented only represent the data from patients with $O A B$. Both the duration of a session and the rate of diuresis during a session (table 1) were not significantly different throughout all sessions (all $p \geq 0.646$ and $p \geq 0.729$ respectively). Despite of the water loading protocol all patients and volunteers state that the (pattern of) bladder sensations experienced during the focus group sessions are comparable with sensations experienced at home. 
Do patients with $O A B$ experience bladder sensations in the same way as healthy volunteers? A focus group investigation

\section{Terminology}

Participants found it hard to describe their bladder sensations. Care was taken to stimulate them to describe sensation in their own words and only vague possibilities, like pleasant or painful, were given as examples. After discussion among the participants all the terms they spontaneously mentioned during the first session, could be brought back to two denominators: pressure and tingling. Four patients experienced only a pressure while six patients not only experienced a pressure but also experienced a tingling sensation during bladder filling. The sensation of pressure remained perceived until the bladder was emptied and was described a continuous sensation. The tingling sensation was not described as continuous, while it could appear and disappear during the same bladder filling. Both sensations could be either felt in the abdomen, the genitalia (openis, qvagina and/or labia) or both locations at the same time. For comparative purposes the Dutch and corresponding English words can be found in table 1 of the previous article on focus group research in healthy volunteers ${ }^{2}$.

The volume at which a first sensation was noted as well as its intensity and the mean voided volume at absolute need to void (table 1) were not significantly different between the three sessions (all $p \geq 0.783, p \geq 0.637, p \geq 0.999$ respectively).

All participants agreed to the proposed consensus terminology though, in daily life and during the focus group session, many of them do not experience all phases during each bladder filling. Which phases they experience can be different for each bladder filling. Similarly to the healthy volunteers ${ }^{2}$, all $O A B$ patients agreed that they can all address their bladder sensations at any time during one bladder filling and therefore interpret the development of an absolute need to void as continuous.

A weak desire to void, if experienced, can temporarily be ignored, though this requires some effort and the ability to concentrate on something else is decreased. A stronger desire to void cannot be displaced from mind. In contrast to the volunteers, all patients, when experiencing the absolute need to void, were unable to complete the evaluation forms, ran to the toilet, were sometimes unable to close the door, sometimes already lost some drops of urine and had to fill out the forms after voiding. Incontinence did not influence the way in which the absolute need to void was experienced. According to the patients descriptions, this absolute need to void resembles urgency as described by the ICS terminology ${ }^{5}$ in only two patients where the absolute need develops suddenly. In $5 / 10$ patients the absolute need develops slowly with a steeper increase in intensity at the end of bladder filling. Remarkable, 3 patients reported that the absolute need to void can develop either suddenly or progress more slowly with a steeper increase in intensity at the end.

\section{Diuresis rates and calculated bladder volumes}

The average natural forced diuresis was significantly lower for patients than for volunteers $(\mathrm{n}=11)$ (mean 6.90, SD $2.8 \mathrm{ml} / \mathrm{min}$ vs. mean 12.1, SD $3.4 \mathrm{ml} / \mathrm{min} ; \mathrm{p}<0.001)$.

The mean development of bladder sensation for the patient and volunteer groups as well as the individual curves for session three are shown in figure 1 and 2 . The curves of the other sessions show similar results. The slope of the curve, which represents the velocity of sensation change, was significantly steeper for patients compared to volunteers 
$(p=0.005)$. The average recalculated volume at the first sensation was significantly lower for patients than for volunteers (mean 91.00 , SD $98.25 \mathrm{ml}$ vs. mean 218.99 , SD 143.29 $\mathrm{ml} ; \mathrm{p}=0.029$ ) while the intensity at the first sensation was significantly higher for patients than volunteers (mean 26.67, SD $23.80 \%$ vs. mean 8.64 , SD $4.94 \%$; $\mathrm{p}=0.041$ ).

Table 1 Shows the detailed information on the duration, diureses and volumes of all sessions for patients $(P)$ and volunteers $(V)$. For each individual the voided volume and the duration of a session were used to calculate individual diuresis rates. These were used to recalculated the bladder volume at each time point. The first sensation is the point that a subject rises above $0 \%$ intensity on the $\mathrm{Y}$-axis for the first time.

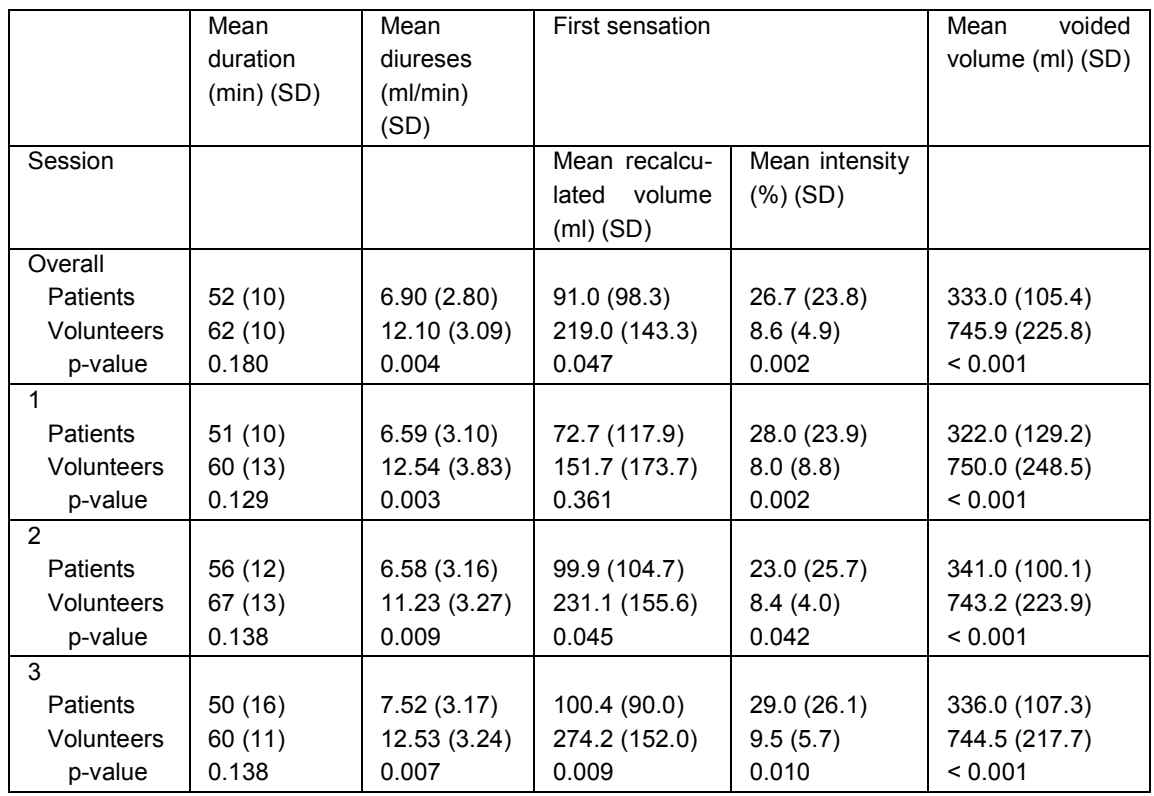

Figure 1 The introspection curves of the third session for all patients and all volunteers. For each individual the voided volume and the duration of a session were used to calculate individual diuresis rates. These were used to recalculated the bladder volume at each time point.
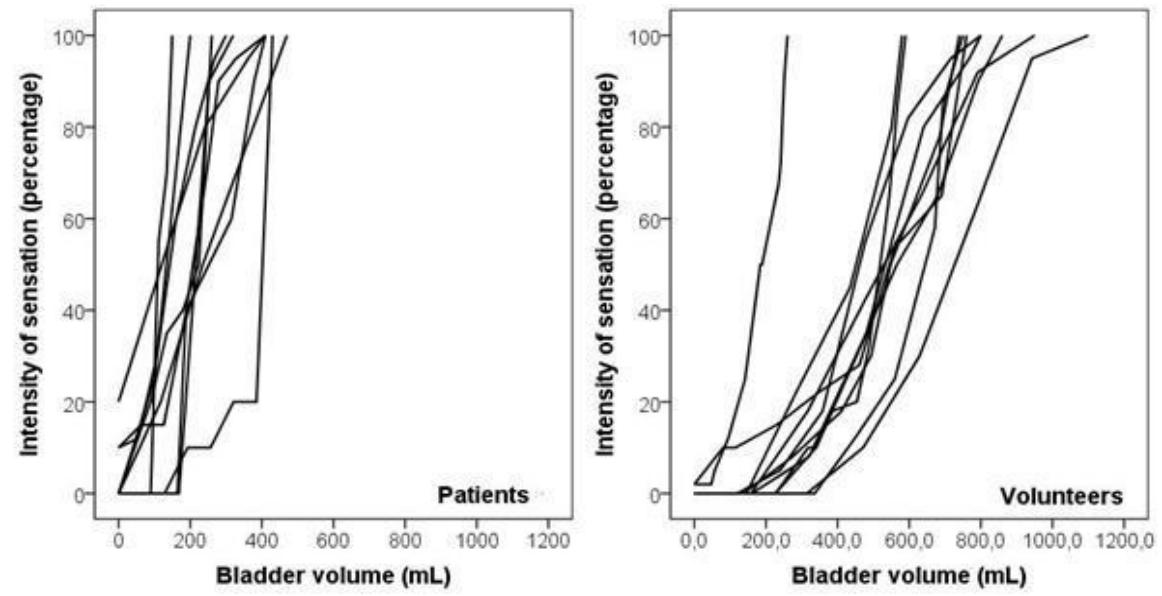
Figure 2 The overall introspection curve for each subgroup. The difference between the overall curve of patients and volunteers is statistically significant with $p=0.005$.

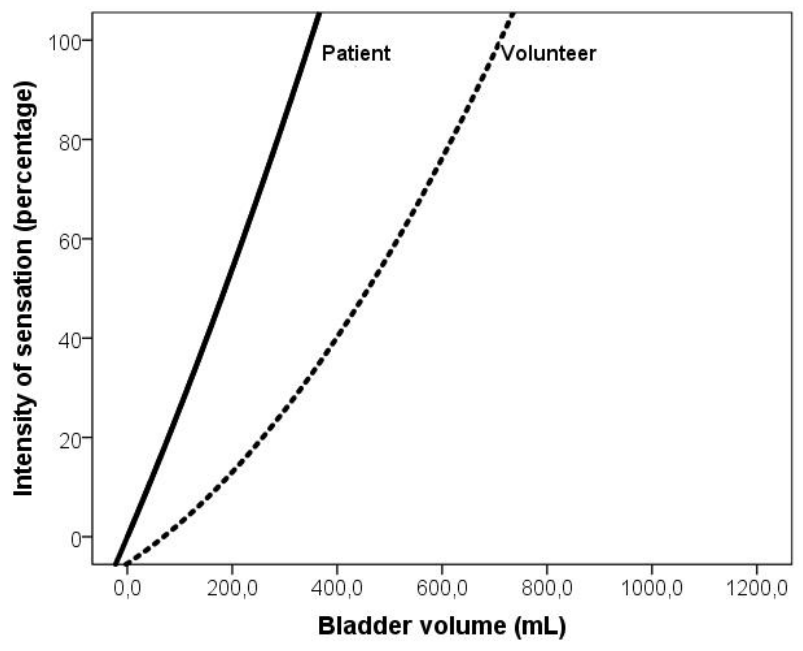

\section{Discussion}

Recently, focus group research has been used to describe normal bladder sensations ${ }^{2}$. In order to evaluate possible differences in bladder sensations in normal and pathological conditions we repeated the identical focus group protocol in patients with OAB.

Regarding terminology, the focus group studies have shown that there are two distinct bladder sensations: a pressure and a tingling sensation ${ }^{2}$. The pressure is the main sensation, experienced by all healthy volunteers and patients with $O A B$, which will gradually build up in intensity, trigger voiding and is, when focused on the bladder, continuously experienced. The tingling sensation is not perceived by all subjects and, although constantly focused on the bladder, this sensation can wax and wane during the same bladder filling. It is mainly felt in the beginning or the end of bladder filling. This tingling sensation appears similar to what has been described urodynamically as 'first sensation of filling, ${ }^{, 1,6}$. The waxing and waning characteristic was then considered to be dependent from cortical fluctuation in the appreciation and interpretation of a weak sensation ${ }^{7}$. In our study however, this tingling sensation can also be experienced at the end of the bladder filling and can therefore not be considered due to its weak nature.

It is the OAB patients' perception, supported by the previously published volunteers perception, that the pattern of sensation development might be better described as continuous rather than distinct episodic sensations as proposed by the ICS terminology paper $^{5}$. Moreover both $\mathrm{OAB}$ patients and healthy volunteers regard the following more differentiating terminology appropriate: no sensation, weak awareness, stronger awareness, weak desire, stronger desire and absolute need. The two terms, pressure and tingling, are not related to this pattern but purely describe the characteristics of the bladder sensations. This means that either phase in the pattern of sensation development can be a pressure, a tingling sensation or both. This study shows that patients and volunteers use the same terms to describe bladder sensations. 
Digesu and colleagues already addressed that, although the duration and localization of bladder sensation depends on the urodynamic diagnosis, the character of the sensation is not significantly different for the studied patient groups ${ }^{8}$. Most women in the study of Digesu et $\mathrm{al}^{8}$ described their bladder sensation as a constant pressure which was confirmed in our study. These findings are important because they implicate that the differences in bladder sensation between healthy subjects and patients are not related to the character of the sensation. This further suggests that urgency might not be a pathological sensation but an intensification of a normal desire to void that patients as well as healthy subjects can experience.

Animal studies in the rat show that there is no difference between activity characteristics of afferent fibres in the hypogastric and pelvic nerve or in myelinated and unmyelinated fibers $^{9}$. During the filling phase, there is a continuously present afferent firing with variable amplitude which represents the 'afferent noise ${ }^{\prime 10}$. The changes in this activity can be used by the central nervous system to 'measure' bladder fullness and be translated into sensation. These data support the 'intensity theory' that states that various visceral sensations are elicited by different activity patterns of one homogenous population of afferent fibres, rather than the 'specificity theory' that proposes that different populations are responsible for different sensations ${ }^{11,12}$. From a clinical point of view, this would suggest bladder sensation to be continuous, as described previously by healthy volunteers ${ }^{2}$ and by $O A B$ patients in the current study, as well as urgency to be an intensification of a normal urge to void. However, the exact processing and translation of the afferent activity towards sensation to trigger voiding is still largely unknown ${ }^{10}$.

Urgency is currently considered pathological and defined by International Continence Society (ICS) as "... a sudden compelling desire to void that is difficult to defer". However, this definition has been debated ${ }^{13-15}$. Some patients that experience urgency, state that it is a sensation different from the normal filling sensations, whereas others experience urgency more as an intensification of the normal sensation and thus not as a simple on-off switch ${ }^{13}$. During cystometric bladder filling, urgency appears sudden in some patients, whereas in others, it is preceded by the normal bladder filling sensations and appears as a continuum ${ }^{15}$. These data could suggest that urgency appears in different 'types', which is also suggested by our study. Urgency can, in patients and volunteers, develop sudden, more slowly progressive or in either of the two ways. Moreover patients and volunteers use the same descriptions of urgency. That volunteers state that urgency can develop suddenly when for example they stopped an activity that they were first intensely focused on, strongly suggests an important central/brain impact on the perception of bladder sensation, and indicates that healthy volunteers have the ability to suppress the awareness of their bladder sensations. This may be the big difference with patients with $O A B$. The latter stated that suppressing a stronger desire to void is difficult and can hardly ever be put out of mind completely. This can be linked to the recently introduced possibility of central modulation of sensory information ${ }^{10}$. This concept entails that sensations generated during a controlled bladder filling are continuous and are the basic pattern of perception of bladder sensation. Healthy volunteers may have the ability to choose which information will reach a conscious level while patients may lack this ability. 
Do patients with $\mathrm{OAB}$ experience bladder sensations in the same way as healthy volunteers? A focus group investigation The overall introspection curves (figure 2) show that bladder sensation develops differently for volunteers and $O A B$ patients. In $O A B$ patients sensation starts at lower recalculated bladder volumes and the intensity of the sensation progresses more rapidly. This is in agreement with urodynamic data ${ }^{16}$.

Unexpectedly and despite the water loading protocol, we found a significant difference in the diuresis between patients and volunteers. This difference suggests a negative fluid balance in $O A B$ patients, which could be due to lower fluid intake in order to reduce complaints although the difference could also be due to a dissimilarity in renal function. Currently the influence of diuresis rate on bladder sensation is unknown. Data using different filling rates during cystometry show that larger volumes can be retained in the bladder before the bladder is considered full at higher filling rates ${ }^{17}$. Post hoc analysis showed that being a patient or a volunteer and diuresis are both independent factors related to recalculated bladder volume ( $p<0.001$ and $p=0.020$ respectively). This means that after correcting for diuresis, there would be a significant difference in bladder volume between patients and volunteers (mean difference $=326.8 \mathrm{ml}, 95 \% \mathrm{Cl} 61.4-592.3$, $\mathrm{p}<0.001$ ). The difference in overall introspection curves (figure 2) can therefore not solely be due to the difference in diuresis, but more research on the difference in diuresis and its possible influence on sensation is needed.

The study has the limitation that all participants are highly educated. We are aware that people with lower levels of education might describe their sensations differently. Because the focus group approach is the first study of its kind in the evaluation of bladder sensation terminology, we have chosen a population that was expected to be capable of accurately describing their sensations. All participants mentioned that compared to daily life, they felt sensations more rapidly during the session, because of the intense focus on their bladder. Although other factors such as age, posture, activity level, bowel status, drinking behaviour, hydration status and other possible pathology (for example chronic pelvic pain syndrome or the painful bladder syndrome) might also influence how bladder sensations are perceived. It would be interesting to address the influence of these factors in a larger diverse cohort. Although the control group is significantly younger than the group of patients, we have chosen these volunteers of younger age because the incidence of pathology influencing bladder sensations is higher at older ages.

\section{Conclusions}

Patients with $\mathrm{OAB}$ complaints experience and describe bladder sensation in the same way as healthy volunteers. However, the volume at which the first sensation is perceived is lower in $O A B$ patients and the velocity of sensation increase is higher. Both in the groups of patients and in volunteers, there seem to be two types of urgency: a sudden absolute need to void and a slowly developing absolute need to void.

\section{References}

1. Wyndaele, J. J.: The normal pattern of perception of bladder filling during cystometry studied in 38 young healthy volunteers. J Urol, 160: 479, 1998

2. Heeringa, R., De Wachter, S. G. G., van Kerrebroeck, P. E. V. A. et al.: Normal Bladder Sensations in Healthy Volunteers: A Focus Group Investigation. Neurourol Urodyn, in press

3. Morgan, D. L.: The Focus Group Guidebook. London: Sage Publications, p. 103, 1998 


\section{Chapter 6}

4. Heeringa, R., van Koeveringe, G. A., van Kerrebroeck, P. E. V. A. et al.: Forced diuresis as a tool for the noninvasive evaluation of bladder sensation. Neurourol Urodyn, 29: 909, 2010

5. Abrams, P., Cardozo, L., Fall, M. et al.: The standardisation of terminology of lower urinary tract function: report from the Standardisation Sub-committee of the International Continence Society. Am J Obstet Gynecol, 187: 116, 2002

6. Wyndaele, J. J., De Wachter, S.: Cystometrical sensory data from a normal population: comparison of two groups of young healthy volunteers examined with 5 years interval. Eur Urol, 42: 34, 2002

7. Denny-Brown, D., Robertson, E. G.: On the physiology of micturition. Brain, 56: 149, 1933

8. Digesu, G. A., Basra, R., Khullar, V. et al.: Bladder sensations during filling cystometry are different according to urodynamic diagnosis. Neurourol Urodyn, 28: 191, 2009

9. Shea, V. K., Cai, R., Crepps, B. et al.: Sensory fibers of the pelvic nerve innervating the Rat's urinary bladder. J Neurophysiol, 84: 1924, 2000

10. Gillespie, J. I., van Koeveringe, G. A., de Wachter, S. G. et al.: On the origins of the sensory output from the bladder: the concept of afferent noise. BJU Int, 103: 1324, 2009

11. Bahns, E., Ernsberger, U., Janig, W. et al.: Functional characteristics of lumbar visceral afferent fibres from the urinary bladder and the urethra in the cat. Pflugers Arch, 407: 510, 1986

12. De Wachter, S.: Afferent Signaling From the Bladder: Species Differences Evident From Extracellular Recordings of Pelvic and Hypogastric Nerves. Neurourol Urodyn, In press

13. Blaivas, J. G., Panagopoulos, G., Weiss, J. P. et al.: Two types of urgency. Neurourol Urodyn, 28: 188, 2009

14. De Wachter, S., Hanno, P.: Urgency: all or none phenomenon? Neurourol Urodyn, 29: 616, 2010

15. De Wachter, S., Wyndaele, J. J.: How sudden is a compelling desire to void? An observational cystometric study on the suddenness of this sensation. BJU Int, 101: 1000, 2008

16. Van Meel, T. D., Wyndaele, J. J.: Reproducibility of urodynamic filling sensation at weekly interval in healthy volunteers and in women with detrusor overactivity. Neurourol Urodyn, (epub ahead of print): doi: 10.1002/nau.21100, 2011

17. Robertson, A. S., Griffiths, C. J., Ramsden, P. D. et al.: Bladder function in healthy volunteers: ambulatory monitoring and conventional urodynamic studies. Br J Urol, 73: 242, 1994 
General discussion 
Normally we are not continuously and consciously occupied by our sensations. Most of the times we get aware of our sensations when they are abnormal, for example sensations of pain or cold. This is also the case for sensations regarding our bladder. If there are no problems related to voiding, voiding is only initiated at a suitable moment without consciously thinking about it. This is not the case for patients with an overactive bladder $(\mathrm{OAB})$. These patients experience abnormal bladder sensations and this influences their voiding behaviour.

Bladder sensations can be evaluated by different means, for example by using the diagnostic tools (1) cystometry and (2) sensation-related bladder diary (SR-BD). In order to be able to study patterns of sensation in pathology it is essential to accept a normal pattern of sensation and the terminology that can be used to describe this. Studies have to be designed so that the terminology used is precise and robust in order to be able to differentiate filling sensation characteristics. Only then, the sensation can be characterised as episodic or continuous and the location of the sensation can be assessed. In this thesis the focus is on (1) describing, developing and refining new methods to evaluate bladder sensation and (2) evaluating the differences in bladder sensations between patients with $O A B$ and healthy volunteers. This chapter presents an overview of the methods used in this and other studies to evaluate bladder sensation. Furthermore it describes a comparison of the terminology used to describe bladder sensations in our studies and in the literature currently available on this issue.

\section{Methods to evaluate bladder sensation}

\section{Sensation related bladder diary}

A bladder diary is a non-invasive tool that can be used to evaluate lower urinary tract function in daily life ${ }^{1}$. Daytime frequency, nocturia, maximum, minimum and mean voided volumes and fluid intake can be determined ${ }^{2}$. In order to be able to study bladder sensations in daily life during a physiological bladder filling, the SR-BD was introduced ${ }^{3}$. Before the introduction of the SR-BD bladder sensations were evaluated by means of cystometry. This technique has the disadvantage of being invasive and filling the bladder in a non-physiological manner. Using the SR-BD it became possible to evaluate bladder sensation while the bladder was filled in the natural way while not only the volume of each void and possible incontinence was noted, but subjects were also asked to grade their degree of urge by a predefined scale ranging from "no sensation" to "need to void now". The combination of the grade of desire and the corresponding voided volume was considered a tool to evaluate bladder sensation. It was found that the mean voided volumes, at every degree of urge, were significantly different and that there was a correlation between the voided volume and the degree of urge $e^{3-5}$. After the introduction of the new scale it has been used and adjusted by several authors ${ }^{6-8}$. After the modification of the degree of urge scale by others, there are now several scales in use, for example the original degree of urge scale ${ }^{3}$, the Patient's Perception of Intensity of Urgency Scale (PPIUS) $^{8}$ and the Urinary Sensation Scale. Although all scales are quite similar, there is no uniformity and therefore studies using these different scales are difficult to compare. A standardised, validated scale used in all studies is preferable. We used a 4-point urge scale with the following options: 0 - no desire to void; 1 - normal desire to void; 2 - 
strong desire to void and 3 - need to void now. An extra option indicating urgency incontinence, as added for example in the PPIUS ${ }^{8}$, is not necessary to our opinion, because the SR-BD already has a column to note any amount incontinence before each void. Furthermore any case of stress incontinence cannot be noted on these types of SR-BDs. To our opinion, a four-point urge scale as used in our studies, is most ideal, because the subjects can relate all possible degrees of urge to one of the four points on the scale. In addition, incontinence before a void, either due to stress incontinence or urgency incontinence, can be filled out in a different column.

Neurophysiological studies in animals have shown that afferent activity is sent up the spinal cord to the brain stem, where bladder filling/volume is measured continuously ${ }^{9}$. From that point of view, the ideal scale to grade bladder volume would be a continuous one. Therefore, apart from the known 4 point urge-scale, we chose a continuous VAS scale to record bladder fullness sensation as a measure for the bladder volume. This measure is further linked to measured voided volumes and the established urge scale is used as a measure of the bladder sensation present before the void. In chapter 3 we showed that the reliability of both the urge measure and fullness VAS measure is good to excellent, except for the voids graded as almost empty (fullness 1) and without sensation (degree of urge 0 ). These values are in agreement with the reliability measures of the urge scale alone previously published for patients with urgency, stress and mixed urinary incontinence ${ }^{10}$.

While measuring voided volumes with a measuring cup can be difficult to combine with daily life, it would be ideal if an urge or fullness scale could replace this. In that case subjects would only have to fill out a scale instead of measuring the voided volume, to get information about voiding behaviour. Analyses show however that, although both scales are reliable and have a positive correlation with bladder volume, both sensory scales cannot replace measuring voided volumes due to a poor agreement between these scales and the actual voided volume. Even a combination of both scales cannot replace the measurement of the voided volume. It would be interesting to see if other researchers can confirm this finding or whether there is a different scale or method that is able to replace measuring voided volumes. It remains to be determined whether either the scales or the measured volumes are influenced by certain therapies for voiding or storage disorders. It is very likely that the use of these scales might give valuable extra information during clinical studies on such therapies.

Although the sensory scales described above cannot replace measuring voided volumes, the combination of both scales can give us information on how a person perceives different bladder volumes. Individual readings of our data show that two extreme types of combinations are possible: the sensation of a relative full bladder with urgent need to void and the sensation of a relative empty bladder with urgent need to void. For example, the combination of a voided volume of $20 \%$ of the maximum bladder capacity, urgent desire to void (urge scale 3 ) and nearly empty bladder (VAS fullness scale 1) may indicate a "psychological" cause such as emotional disturbances or nervousness providing a different perceptual threshold. On the other hand the voided volume of $20 \%$ of the maximum bladder capacity, urgent desire to void (urge scale 3) with a full bladder (VAS fullness scale 9) may point to a "physiological" bladder disorder such as detrusor overactivity. This is an interesting observation because it can identify two types of urgency. Stud- 
ies will have to show, whether these different types of urgency might each benefit from other treatment options.

The finding that the relation between bladder volume and sensation is more complex than a simple linear relation is also supported by brain imaging studies. These studies have shown that different brain regions are involved in the registration of either bladder filling/volume or bladder sensation and that these regions can be disturbed or modulated independently ${ }^{11-13}$. Through a brain imaging study, Griffiths and colleagues also showed that bladder control in subjects without $O A B$ is different from patients with urgency incontinence11. In $O A B$ patients cortical responses become amplified at large bladder volumes and strong sensation, but without actual detrusor overactivity. According to the authors this might be due to both a learning reaction to previous incontinence episodes and the neural correlate of urgency. Recently, a different research group showed that, compared to healthy subjects, patients with $O A B$ have an increased brain activation in the limbic cortex ${ }^{1} 4$. They conclude that this activation is likely to represent an abnormal processing of sensory input in brain regions associated with an emotional response to discomfort.

\section{Water loading protocol}

Cystometry has two major limitations: it is an invasive procedure, the bladder has to contain catheters to do so, and the bladder is filled in a non-physiological manner. Therefore, we introduced a water loading protocol. This gave us the opportunity to evaluate bladder sensations while the bladder filled in a rapid, non-invasive bladder way. Furthermore, due to a constant high diuresis, a controlled bladder filling could be achieved. Whether it is a physiological bladder filling after a load of 2 litres of water in 2 hours can be argued. For sure, the bladder is filled by a natural route. Furthermore this option was chosen in order to have a controlled natural bladder filling with a predictable maximum constant diuresis, to make an objective evaluation of sensations possible.

Although there is a large variability between subjects, we have shown that the water loading protocol induces a constant diuresis per individual per session. Between the sessions the diuresis can differ only slightly. This can be due to the fact that diuresis is not only dependent on fluid intake but also on renal function, dietary proteins and hydration level ${ }^{1} 5$. We also found that $O A B$ patients had a significantly slower diuresis than healthy volunteers. This difference suggests a negative fluid balance in $O A B$ patients due to long standing reduction in fluid intake in order to reduce symptoms. Another hypothesis is that an overactive bladder does lead to changes in the fluid balance of the body for example through a decreased sensation of thirst. Whether or if so, how this difference in diuresis influences the sensation development is not clear. An ad hoc analysis however, showed that being a patient or a volunteer and diuresis are both independent factors in the relationship to bladder volume. The difference in overall introspection curves, as discussed in the next paragraph, can therefore not solely be due to the difference in diuresis. More research on the difference in diuresis between healthy volunteers and patients with $O A B$ and the possible influence of diuresis on bladder sensation is definitely needed. 


\section{Introspection curve}

Whether the pattern of development of normal bladder sensations is episodic or continuous is being debated. In our studies we found that the sensation of an absolute need to void develops in a continuous manner for both patients and volunteers. We studied the entire course of the development of an absolute need to void by asking participants to grade the intensity of their bladder sensations during a water loading protocol at regular time points on an empty graph. The $\mathrm{X}$-axis was an open end time scale and the $\mathrm{Y}$-axis the intensity of the sensation ranging from $0 \%$ indicating no sensation to $100 \%$ indicating the absolute need to void. Healthy volunteers have drawn two general patterns of "focused introspection" curves: a convex and a S-shaped curve (see figure 2 of chapter 4). Within an individual, the curves show a good agreement during the three sessions. In accordance with earlier studies on bladder sensation during cystometry ${ }^{16}$, we found that the "first sensation of filling" appears to be the most variable one possibly due to level of focus or attention towards bladder filling. Similar sensation curves are described during cystometric bladder filling ${ }^{17}$. However, due to the invasive character of the cystometry, we believe the water loading protocol is the best option to evaluate the pattern of development of bladder sensations. Eighteen of the twenty five curves described in that study were linear, whereas only 7 were "non-linear" and resemble the curves from our present study. Data from afferent animal recordings ${ }^{18}$ show that, at physiological filling rate, a variety in afferent response patterns exists. This variety disappears if the filling rate is increased to a supraphysiological rate. Therefore the high non physiological filling rate is likely to cause the "linear" curve. We hypothesize that if the bladder is filled with lower filling rates the curves will become less linear and therefore resemble the curves that we found in this study. This hypothesis, however, has to be confirmed by additional studies. By looking at the introspection curves we see that bladder sensations develop significantly different in $O A B$ patients compared to volunteers: in OAB patients, sensation starts at lower bladder volumes and the intensity of the sensation progresses more rapidly (e.g. steeper line, steeper slope). Although this difference has been suggested before ${ }^{1} 9$, our study, using forced diuresis, is the first to demonstrate this difference in sensation development in a non-invasive way.

\section{Terminology}

\section{Development of terminology to assess sensation}

The current terminology used to assess bladder sensation in not optimal, therefore in this thesis, a serious attempt was made to revisit current terminology. The obvious place to start is right back at the beginning: simply ask subjects with lower urinary tract symptoms to describe what they feel as their bladder fills. This, however, is easier said than done. Particularly early in the filling phase, it is not obvious to a naïve subject what specific sensation he or she is being asked to focus on.

Both the terminology that was used until now to describe normal and pathological bladder sensations and the techniques during which the terminology came about have some disadvantages. The most important disadvantages are the possible influence of the researcher on the results and the fact that the terms do not mean the same to all patients and/or clinicians. Using questionnaires in order to develop such terminology is difficult 
because open ended questions will lead to many different answers and closed ended or suggestive questions will force the subject to relate their own sensations to terminology used by the examiner. In the field of psychology, focus group research is often used to evaluate ideas, opinions especially on the assessment of bodily signals. This is an iterative process of individuals that discuss on a specific subject. By means of discussions between the subjects, participants can be stimulated to describe certain topics and give examples. Ambiguities can be clarified directly as there are often more sessions with the researcher present. Another advantage of this method is that there is an interaction between all participants and a specific participant might come up with ideas or examples as a result of the comment of a different participant. During our pilot focus group sessions with healthy volunteers and patients with $O A B$, the interaction was considered a positive experience by all subjects. They estimated the group discussion valuable as a means to better formulate their own opinion. In our final method design we choose to combine focus group discussions with the filling out of different questionnaires. The discussions were merely used in order to clarify the terminology regarding bladder sensations, while the questionnaires were used to assess the location of sensations and to evaluate the pattern of the development of bladder sensations. Taking into account the above mentioned, we organised focus group sessions with both healthy volunteers and patients with overactive bladder symptoms in order to evaluate the terminology in relation to pathology. All subjects, healthy volunteers and patients, used different words to describe their bladder sensations but all these words could be brought back to two denominators: pressure and tingling. Pressure is the main sensation which builds up gradually in intensity, triggers voiding and is continuous. It is perceived in the lower abdomen, genitalia or at both locations at the same time. The term pressure has been used previously to describe a continuous bladder sensation ${ }^{7}$. Some participants experience a secondary tingling sensation that can or cannot be perceived at the same time as the pressure sensation. This tingling sensation does not have a continuous pattern and is perceived mainly at the beginning or at the end of the bladder filling. The tingling sensation appears similar to what has been described urodynamically as 'first sensation of filling' 20 . The waxing and waning characteristic was then considered to be dependent on cortical fluctuations in the appreciation and interpretation of a weak sensation ${ }^{21}$. In our study however, this tingling sensation can also be experienced at the end of the bladder filling and while sensation is intense at that point in time, the sensation can therefore not be considered to wax and wane due to its weak nature. Digesu and colleagues already addressed that the character of the sensation is not significantly different for the studied patient groups ${ }^{7}$, although the duration and position of bladder sensation depends on the urodynamic diagnosis. Most women in the study of Digesu et al. ${ }^{7}$ describe their bladder sensation as a constant pressure, which is in agreement with our findings. These findings are important because they imply that bladder sensations in healthy subjects and patients are better differentiated by both the time course and the amplitude of the sensation compared to the character of the sensation. 


\section{How does bladder sensation reach our mind?}

\section{Patterns of development of both sensation and urgency}

According to the ICS guidelines ${ }^{2}$, the pattern in which the absolute need to void develops, is described by the terms: first sensation, normal desire and strong desire. During the focus group sessions however, volunteers and patients better relate to the following terms: no sensation, weak awareness, stronger awareness, weak need, stronger need, absolute need. There seem to be two patterns to reach the absolute need to void: slowly progressing while all steps in the pattern of sensation development are experienced as mentioned above or no or only little sensation during the initial period followed by a sudden need while all or several steps of the pattern described above are skipped. These patterns better describe the continuous manner in which the sensation will develop up to the absolute need. Evidence for bladder sensations to develop in a continuous pattern, instead of an episodic pattern, can also be found in studies done in the rat, that show that there is no difference between activity characteristics of afferent fibres in the hypogastric and pelvic nerve or in myelinated and unmyelinated fibers ${ }^{15}$. During the filling phase, there is a continuous afferent firing, which varies in amplitude and represents the 'afferent noise ${ }^{22}$. The amplitude changes of this activity can be used by the central nervous system to 'measure' bladder fullness and can further be translated into a conscious sensation. These data support the 'intensity theory' that states that various visceral sensations are elicited by different activity patterns of one homogenous population of afferent fibres, rather than the 'specificity theory' that proposes that different populations of fibres are responsible for different sensations ${ }^{23,24}$. From a clinical point of view, this would confirm the continuous nature of the bladder sensation. However the exact processing and translation of the afferent activity towards sensation to trigger voiding is still largely unknown ${ }^{22}$.

In five OAB patients the absolute need to void has been shown to develop slowly with a steeper increase at the end of the bladder filling, in 2 patients urgency always develops suddenly and in 3 patients urgency can develop either slowly or suddenly. These descriptions of urgency were also used by healthy volunteers. In 2008 de Wachter et al. described that the absolute need to void can develop via different patterns in patients with urgency and detrusor overactivity ${ }^{25}$. This study showed that a compelling desire to void (CDV) can develop (1) suddenly if normal filling sensation is disturbed $(13 \%$ of the subjects) or (2) gradually if normal filling sensation is preserved (66\% of the subjects). In cases of disturbed filling sensations, they found that the volume at a compelling desire to void and the warning volume were significantly lower. Blaivas et al. also suggested the presence of two types of urgency, although they differentiate the types by the characteristic of the sensation ${ }^{26}$. Patients complaining of urgency described in $68.8 \%$ the urge sensation as being an intensification of the normal sensation and in $31.1 \%$ as being a different sensation. The patients and healthy volunteers in our focus group sessions did not experience this difference.

Participants that usually had a slowly progressive pattern of urge increase, recognized from personal experience, a change in development of urge when they stopped an activity that they were first intensely focused on. This may indicate that healthy volunteers have the ability to suppress the awareness of their bladder sensations and this might in 
the future prove to be a significant difference with patients with $\mathrm{OAB}$. The latter stated that suppressing a stronger desire to void is very difficult and can hardly ever be removed from the mind completely. This can be linked to the recently introduced hypothesis of central modulation of sensory information ${ }^{22}$. This concept entails that sensation information is continuously available and can be assessed at every desired moment. There seems to be a hierarchy in sensations in which one category can be displaced from the mind and another category cannot or can with only strong mental effort. Displacing certain sensations from the mind has shown to be possible for example in relation to pain. Studies showed that ignoring pain can help to reduce the degree to which pain interferes with the ability to interfere with for example everyday work, social life and sports $^{27,28}$. Figure 1 shows the relationship between and hierarchy of the structures involved in experiencing sensations. Healthy volunteers may have the ability to choose which information will reach a conscious level based on the characteristics of the sensation. In other words a healthy subject can control (in a conscious or unconscious manner) which information in part $C$ will reach part $B$. Based on the sensation that is experienced, the subject can choose to act upon the sensation or not (part $A$ ). Patients with $\mathrm{OAB}$ may lack this ability meaning that every sensation that will reach part $C$ will also reach part $B$. Depending on the experienced sensation the subject will act upon it. If the selection of which information will reach a conscious level can be trained (influencing the relation between part $C$ and $B$ in figure 1 ), than this may very well be a non-invasive new treatment option, from which patients with $O A B$ may benefit.

Figure 1 shows the relationship between and hierarchy of the structures involved in experiencing any bodily sensation. For example the bladder (part E) is full and signals this through the nerves in the spinal cord (part D) to the micturition centre in the brain (part $C$ ). If this sensation is strong enough it will reach the consiousness of the subject (part B) and this will lead to searching in toilet to empty the bladder (part A).

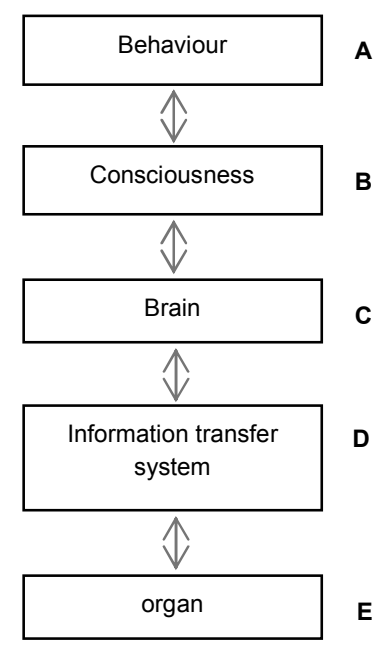




\section{Answers}

In the Aims section of this thesis, a series of questions were raised. Underneath are the answers to these questions summarized that came about in both the thesis chapters and the general discussion.

1. Can bladder sensations be studied during a controlled non-invasive bladder filling by means of a water loading protocol?

Bladder sensations can be studied during a controlled non-invasive bladder filling. A water loading protocol, as used in our studies, has been shown to induce a constant high diuresis. Therefore the bladder filling occurs in a controlled manner. When a subject fills out an introspection curve (as described in chapter 4) during the water loading protocol, the bladder sensations of this subject are being assessed in a non-invasive manner.

2. Which words do healthy volunteers or patients with $O A B$ use to describe bladder sensations?

Healthy volunteers and patients with $O A B$ use different words to describe their bladder sensations. All these terms can be brought back to two denominators that describe the sensations experienced during a bladder filling. The main sensation, experienced by everyone, is the sensation of pressure. The other sensation, is a tingling sensation.

3. How does the absolute need to void develop in healthy volunteers and patients with the overactive bladder syndrome (continuous or episodic)?

The introspection curves, as described in chapter 4 and 6, show that, if a subject is constantly focused on his or her bladder, the absolute need to void develops in a continuous way.

4. Can grading of the perception of bladder fullness or the degree of urge replace measuring voided volumes on a sensation-related bladder diary?

Although the perception of bladder fullness and the degree of urge scale are reliable to use and both scales have a significant relation with bladder volume, these scales cannot replace measuring voided volumes.

5. Are bladder sensations experienced at home different from bladder sensation experienced at the hospital?

Bladder sensations experienced at home differ from sensations experienced at the hospital. Both volunteers and patients experience the intensity of perception of bladder fullness and degree of urge, for the same bladder volume, to be higher at home than during a filling session in the hospital.

\section{Conclusions}

In order to refine the terminology to describe bladder sensations we used the technique of focus group research with a constant focus on the bladder sensations. Healthy volunteers and patients with $O A B$ describe the characteristics of their bladder sensations as a pressure or a tingling sensation (in Dutch: "tintelend gevoel"). We showed that the pattern can be described more in detail by using the terms: no sensation, weak awareness, stronger awareness, weak need, stronger need and absolute need to void. There seem to be two patterns to reach the absolute need to void: slowly progressing while all steps 
in the pattern of sensation development are experienced as mentioned above or no or only little sensation during the initial period followed by a sudden need while all or several steps of the pattern described above are skipped.

To evaluate the development of the absolute need to void in a non-invasive manner, a water loading protocol can be used. During this method a constant high diuresis is induced and combining this protocol with constant introspection offers the opportunity to evaluate bladder sensations during a non invasive, controlled constant bladder filling rate. The absolute need to void can develop according to two different patterns that have been described above, but per individual the pattern of development is constant. The overall pattern of development is significantly different between volunteers and $O A B$ patients. Patients with $O A B$ have a significantly lower diuresis than healthy volunteers, this is suggestive of a negative fluid balance in patients with $O A B$.

Besides the use of focus group research and the introspection curve, bladder sensations can be studied by means of the sensation-related bladder diary. When combining the degree of urge and the perception of bladder fullness on a bladder diary, it was shown that both scales are reliable to use and have a significant relationship with bladder volume in a controlled (hospital) and an uncontrolled (home) setting. The combination of the perception of fullness scale and the degree of urge scale for a given void provides more information than either scale on its own. Usage of these two scales cannot replace the measurement of voided volumes. Bladder sensation in an uncontrolled (home) setting is different from bladder sensation in a controlled (hospital) setting and might be more dependent on less standardized influences from within or outside of the subject, for example the stress level of the subject, the presence of other people or distraction of the subjects attention by daily activities.

\section{Future directions}

In this thesis a new technique to evaluate bladder sensations in a non-invasive manner was introduced. We have shown that this technique is reliable and shows similar results in small test groups. However, in order to validate this method and use it on a larger scale in clinical practice, future studies should focus on three things. We must be sure that the introspection curve is independent of diuresis. We describe a significant difference in diuresis between healthy volunteers and patients with OAB. After correcting for this difference, analyses show that the significant difference between the curves of volunteers and OAB patients still exists. Therefore it is proposed to study the exact effect of diuresis on bladder sensation / introspection curve in a larger group.

In order to validate this new method, it has to be clarified what the possible effect of position on bladder sensation is. Due to gravity the position (lying down, standing, sitting) may induce different sensations. One of the hypotheses in the aetiology of OAB locates the primary disorder in the bladder wall. The bladder wall appears to be composed of different autonomous modules that may be activated during bladder filling independently. These modules may "sample" the amount of urine and eventually give rise to afferent activity that subsequently leads to sensation. Disturbed function of these modules has been linked to the origin of pathological urgency ${ }^{29}$. How these modules are arranged inside the bladder wall is unknown, but dysfunctions in the bladder neck and/or posterior urethra might be the primum novens in the urgency sensation. Histological studies in 
animals clearly show that the sensory axons are not equally distributed in the bladder wall, a dense, multi-branching network of sensory fibres has been seen around the bladder neck ${ }^{30}$ that becomes less dense towards the bladder dome ${ }^{31}$. In humans the different degrees of urge are typically perceived in a referred manner in different body sites: normal desire to void is perceived in the suprapubic region, whereas urgent desire to void and urgency is perceived more prominently in the perineal and genital region ${ }^{20}$. Changes in the perception threshold of the bladder neck have been shown in patients with urgen$\mathrm{cy}^{32}$, while the threshold in the bladder wall is not linked to urge sensations ${ }^{33}$. These data show the importance of the bladder neck in the perception of urge and urgency. In healthy volunteers, it has already been shown that the voided volume after lying down and sleeping during the night is significantly larger than the volume of the largest void produced during the day associated with a similar perception of bladder fullness ${ }^{3}$. The difference in volumes might be due to a difference in body position, although a changed inhibition of bladder perception during sleep cannot be ruled out. The findings above indicate that the possible effect of position on bladder sensations has to be clarified. A pilot study conducted by professor Gillespie et al. (personal communication with professor Gillespie) suggested that patient's postural position indeed has an impact on how patients perceive their bladder fullness. Finally, anecdotal data from patients suffering from incontinence show that a change in body position from lying to sitting or standing, is linked to an increase in urge with increased difficulty to postpone micturition.

Furthermore, a structured evaluation of the exact effect of location on bladder sensation is advisable in a larger group. We evaluated the relationship between bladder sensation experienced in a controlled and an uncontrolled setting by using both scales at home and in the hospital. Analyses show that bladder sensation in an uncontrolled setting is different from bladder sensation in a controlled setting and might be more dependent on influences from within or outside of the subject. However, this study has the limitation that, while comparing bladder sensation at home and in the hospital two different techniques have been used i.e. only grading sensation before a void and regular grading sensation during a rapid physiological bladder filling, respectively. A study design that evaluates bladder sensation during the rapid physiological bladder filling in both settings would be preferable.

\section{References}

1. Abrams, P., Klevmark, B.: Frequency volume charts: an indispensable part of lower urinary tract assessment. Scand J Urol Nephrol Suppl, 179: 47, 1996

2. Abrams, P., Cardozo, L., Fall, M. et al.: The standardisation of terminology of lower urinary tract function: report from the Standardisation Sub-committee of the International Continence Society. Am J Obstet Gynecol, 187: 116, 2002

3. De Wachter, S., Wyndaele, J. J.: Frequency-volume charts: a tool to evaluate bladder sensation. Neurourol Urodyn, 22: 638, 2003

4. Naoemova, I., De Wachter, S., Wyndaele, J. J.: Comparison of sensation-related voiding patterns between continent and incontinent women: a study with a 3-day sensation-related bladder diary (SR-BD). Neurourol Urodyn, 27: 511, 2008

5. Naoemova, I., Van Meel, T., De Wachter, S. et al.: Does sensory bladder function during cystometry differ from that in daily life? A study in incontinent women. Neurourol Urodyn, 28: 309, 2009

6. Honjo, H., Kawauchi, A., Ukimura, O. et al.: Analysis of bladder diary with urinary perception to assess overactive bladder symptoms in community-dwelling women. Neurourol Urodyn, 2009

7. Digesu, G. A., Basra, R., Khullar, V. et al.: Bladder sensations during filling cystometry are different according to urodynamic diagnosis. Neurourol Urodyn, 28: 191, 2009 


\section{Chapter 7}

8. Cartwright, R., Panayi, D., Cardozo, L. et al.: Reliability and normal ranges for the Patient's Perception of Intensity of Urgency Scale in asymptomatic women. BJU Int, 105: 832, 2009

9. Shea, V. K., Cai, R., Crepps, B. et al.: Sensory fibers of the pelvic nerve innervating the Rat's urinary bladder. J Neurophysiol, 84: 1924, 2000

10. Naoemova, I., De Wachter, S., Wuyts, F. L. et al.: Reliability of the 24-h sensation-related bladder diary in women with urinary incontinence. Int Urogynecol J Pelvic Floor Dysfunct, 19: 955, 2008

11. Griffiths, D., Tadic, S. D.: Bladder control, urgency, and urge incontinence: evidence from functional brain imaging. Neurourol Urodyn, 27: 466, 2008

12. Blok, B. F.: Central pathways controlling micturition and urinary continence. Urology, 59: 13, 2002

13. Blok, B. F., Willemsen, A. T., Holstege, G.: A PET study on brain control of micturition in humans. Brain, 120 ( Pt 1): 111, 1997

14. Komesu, Y. M., Ketai, L. H., Mayer, A. R. et al.: Functional MRI of the Brain in Women with Overactive Bladder: Brain Activation During UrinaryUrgency. Female Pelvic Med Reconstr Surg, 17: 50, 2011

15. de Rouffignac, C.: Effects of water balance, diet and antidiuretic-hormone administration on the renal excretion of water. Scand J Urol Nephrol Suppl, 202: 31, 1999

16. Wyndaele, J. J., De Wachter, S.: Cystometrical sensory data from a normal population: comparison of two groups of young healthy volunteers examined with 5 years interval. Eur Urol, 42: 34, 2002

17. Dompeyre, P., Fritel, X., Bader, G. et al.: Bladder sensitivity testing using a visual analogue scale: comparative cystometric study on women. Neurourol Urodyn, 26: 350, 2007

18. De Wachter, S., De Laet, K., Wyndaele, J. J.: Does the cystometric filling rate affect the afferent bladder response pattern? A study on single fibre pelvic nerve afferents in the rat urinary bladder. Neurourol Urodyn, 25: 162, 2006

19. Lee, S. R., Kim, H. J., Kim, A. et al.: Overactive bladder is not only overactive but also hypersensitive. Urology, 75: 1053, 2010

20. Wyndaele, J. J.: The normal pattern of perception of bladder filling during cystometry studied in 38 young healthy volunteers. J Urol, 160: 479, 1998

21. Denny-Brown, D., Robertson, E. G.: On the physiology of micturition. Brain, 56: 149, 1933

22. Gillespie, J. I., van Koeveringe, G. A., de Wachter, S. G. et al.: On the origins of the sensory output from the bladder: the concept of afferent noise. BJU Int, 103: 1324, 2009

23. Bahns, E., Ernsberger, U., Janig, W. et al.: Functional characteristics of lumbar visceral afferent fibres from the urinary bladder and the urethra in the cat. Pflugers Arch, 407: 510, 1986

24. De Wachter, S.: Afferent Signaling From the Bladder: Species Differences Evident From Extracellular Recordings of Pelvic and Hypogastric Nerves. Neurourol Urodyn, In press

25. De Wachter, S., Wyndaele, J. J.: How sudden is a compelling desire to void? An observational cystometric study on the suddenness of this sensation. BJU Int, 101: 1000, 2008

26. Blaivas, J. G., Panagopoulos, G., Weiss, J. P. et al.: Two types of urgency. Neurourol Urodyn, 28: 188, 2009

27. Cano, A., Mayo, A., Ventimiglia, M.: Coping, pain severity, interference and disability: The potential mediating and mederating roles of race and education. The Journal of Pain, 7: 459, 2006

28. Deroche, T., Woodman, T., Stephan, Y., et al.: Athletes' indclination to play through pain: a coping perspective. Anxiety Stress Coping, 24: 579, 2011

29. Drake, M. J., Harvey, I. J., Gillespie, J. I. et al.: Localized contractions in the normal human bladder and in urinary urgency. BJU Int, 95: 1002, 2005

30. Grol, S., van Koeveringe, G. A., de Vente, J. et al.: Regional differences in sensory innervation and suburothelial interstitial cells in the bladder neck and urethra. BJU Int, 102: 870, 2008

31. Gabella, G., Davis, C.: Distribution of afferent axons in the bladder of rats. J Neurocytol, 27: 141, 1998

32. Creighton, S. M., Plevnik, S., Stanton, S. L.: Urethral sensitivity in the aetiology of sensory urgency. Br J Urol, 73: 190,1994

33. De Wachter, S., Wyndaele, J. J.: Can the sensory threshold toward electrical stimulation be used to quantify the subjective perception of bladder filling? A study in young healthy volunteers. Urology, 57: 655, 2001 
Summary \& Samenvatting 


\section{Summary}

The first aim of this thesis is to describe, develop and refine new techniques for the evaluation of bladder sensations. Unfortunately at the moment the diagnostic tools are either invasive (urodynamic research) or do not describe the entire pattern in which an absolute need to void develops (sensation related bladder diary). It is however essential that bladder sensations can be studied by means of a technique that is non-invasive and evaluates the entire pattern of bladder sensations ranging from no sensation to an absolute need to void. Only then, and with the right terminology, we can further unravel the differences in (the development of) bladder sensations between patients and healthy volunteers in daily life.

The second aim of this thesis is to study the differences in bladder sensations between patients with the overactive bladder syndrome $(O A B)$ and healthy volunteers. Individuals without lower urinary tract symptoms are not constantly focused on their bladder sensations and only act upon these sensations if they have an absolute need to void or if they choose to. However, there are individuals for whom the normal pattern of bladder sensations, introspection and behaviour, is altered. One of the pathological conditions in which patients experience altered bladder sensations is the overactive bladder syndrome. Frequency and urgency are the cornerstones of this symptoms complex. While history taking is an important tool to diagnose patients with $O A B$, we do not have a clear and unambiguous terminology to describe normal and pathological bladder sensations. This makes it difficult for patients to describe their symptoms and difficult for the clinicians to understand and correctly diagnose the problem. Having a clear terminology is therefore important in order to further unravel the aetiology of pathological conditions and to prevent misdiagnosis.

Chapter 1 is the introduction of this thesis and describes what is known on normal and pathological bladder sensations and the techniques to evaluate these sensations.

Chapter 2 is a critical evaluation of all the work on bladder sensation and argues the necessity to return to first principles and a re-examination of the problem. We explore the use of focus groups in order to identify relevant bladder sensations and the triggers of "bladder" behaviour. We argue that there are differences in what can be described as "introspective bladder sensations" and the sensations reported immediately before a void, "void sensations." Peripheral information, information from the bladder and urethra, generates "introspective sensations" and "void sensations". We propose an alternative model to describe how these sensations might be different but interrelated. By exploring such ideas and identifying such complexity it is our intention to stimulate debate and generate further research in this field in order to understand better the physiology of bladder sensation and the pathophysiology of increased urge, frequency, and incontinence.

Chapter 3 evaluates the reliability of two sensory scales (the degree of urge scale and perception of bladder fullness scale), the relationship between both scales and bladder volume, and possible differences between bladder sensation experienced in a controlled 
or uncontrolled setting. Results show that both scales are reliable and that each scale is significantly related to bladder volume. The agreement between both scales and voided volume is poor, indicating that the use of these scales cannot replace measuring a voided volume. Patients and volunteers grade their bladder fullness and degree of urge to be higher in an uncontrolled setting than in a controlled setting. Bladder sensations in the hospital might therefore underestimate the bother of the symptoms a patient experiences in daily life.

Chapter 4 introduces a new non-invasive method to study the origin and development of bladder filling sensation and evaluates the repeatability of the method. By means of a constant high diuresis, bladder sensations were evaluated on an empty graph with time on the $\mathrm{X}$-axis and intensity of sensation on the $\mathrm{Y}$-axis. Results show that the diuresis induced with the water loading protocol was not different between the sessions. Two patterns of sensation development were found. In both types, phase 1 represents the silent phase. Phase 2 shows a slowly increasing intensity of sensation and phase 3 a steep rise in intensity. For type 1 this steep rise leads to an absolute need to void. For type 2 a fourth phase is characterized by a slowly increasing sensation intensity, leading to an absolute need to void. For an individual the pattern did not change over the three sessions.

Chapter $\mathbf{5}$ describes the bladder sensations experienced during non-invasive rapid bladder filling in a controlled setting in healthy volunteers by means of focus group research. In order to induce a constant maximum diuresis, a water loading protocol was given to all participants. Healthy subjects describe two types of bladder sensations: (1) a "pressure", which is the main sensation and develops continuously and (2) a "tingling" sensation, which is not experienced by everybody and does not necessarily develop in a continuous manner. The pattern in which the absolute need develops is described by the terms: no sensation, weak awareness, stronger awareness, weak need, stronger need and absolute need to void.

Chapter 6 portrays the terminology and pattern of bladder sensations experienced during non-invasive rapid bladder filling in a controlled setting in patients with $O A B$ and compares these results with the results described in chapter 5. In comparison with healthy volunteers, patients also describe their bladder sensations as a pressure or a tingling sensation while the pattern can be described by terms ranging from no sensation to an absolute need to void. There seem to be two types of urgency: a sudden absolute need to void and a slowly developing absolute need to void. The mean development of bladder sensation is significantly different between patients and healthy volunteers and patients have a significant lower diuresis suggesting a negative fluid balance.

Chapter 7 is a general discussion in which the results and discussions of all articles (chapter 2-6) is combined with information from recent studies by other authors. 


\section{Overall conclusion}

By means of focus group research and constant focus on the bladder, it is possible to describe bladder sensations and to refine the terminology used by healthy participants and patients with $\mathrm{OAB}$. A water loading protocol induces a constant high diuresis and combining this protocol with constant introspection offers the opportunity to evaluate the development of the absolute need to void non-invasively. Besides the use of focus group research and introspection curves, bladder sensations can be studied by means of the sensation-related bladder diary with a degree of urge scale and a bladder fullness scale. In order to validate the techniques described in this thesis and use them in clinical practice, future studies must focus mainly on two aspects. First we have to be sure that the introspection curve is independent of diuresis. Furthermore we must clarify what the exact effect of position and location on bladder sensations is. 


\section{Samenvatting}

Het eerste doel van deze thesis is het beschrijven, ontwikkelen en perfectioneren van nieuwe technieken die het mogelijk moeten maken blaassensaties te onderzoeken. Dit is wenselijk omdat de huidige technieken voor het evalueren van blaassensaties vaak belastend zijn voor de patiënt. Dit komt doordat de meeste onderzoeken een invasief karakter hebben, waarbij er inwendig (urodynamisch) onderzoek plaatsvindt. Bijkomend probleem is dat deze technieken niet het gehele ontstaanspatroon bestuderen waarbinnen deze blaassensaties zich ontwikkelen (sensatie gerelateerd plasdagboek). Het is belangrijk dat het onderzoek naar blaassensaties zo min mogelijk belastend is zodat er minder risico's zijn aan het ondergaan van het onderzoek en er een kleinere drempel is voor patiënten en gezonde vrijwilligers om aan het onderzoek deel te nemen. Een vaste terminologie waarbij het gehele ontstaanspatroon van "geen gevoel" tot een "gevoel van moeten plassen" juist wordt beschreven, kan er dan voor zorgen dat verschillen in het ontstaan van blaassensaties tussen patiënten en gezonde vrijwilligers kunnen worden opgehelderd.

Het tweede doel van deze thesis is het bestuderen van de verschillen in blaassensaties bij patiënten met een overactieve blaas en gezonde vrijwilligers. Individuen zonder klachten van de lagere urinewegen ervaren niet continu sensaties afkomstig van de blaas. Ze ondernemen actie op deze sensaties wanneer ze het gevoel hebben dat ze moeten plassen of wanneer ze dat willen. Er zijn echter ook mensen voor wie dit normale patroon van blaassensaties en gedrag als gevolg van blaassensaties verstoord is. Eén van de afwijkingen waarbij mensen last hebben van veranderde blaassensaties, is het overactieve blaassyndroom (OAB). De belangrijkste symptomen hierbij zijn het vaak moeten plassen (frequentie) en een onhoudbare aandrang (urgentie). Hoewel de anamnese een belangrijk middel is tot het stellen van de diagnose $O A B$, ontbreekt het momenteel aan een duidelijke terminologie voor het beschrijven van normale en pathologische blaassensaties. Dit leidt ertoe dat het voor patiënten moeilijk is om hun symptomen te beschrijven. Voor de artsen is het moeilijk om het beschreven probleem te begrijpen en de juiste diagnose te stellen. Een duidelijke terminologie is dan ook belangrijk indien we de ontstaanswijze van pathologische afwijkingen willen ontrafelen en foute diagnosen willen voorkomen.

Hoofdstuk 1 is de introductie van deze thesis. Het beschrijft wat er, voorafgaand aan het verrichten van de in deze thesis beschreven onderzoeken, bekend was over normale en pathologische blaassensaties. Ook de technieken waarmee deze werden geëvalueerd worden in dit hoofdstuk beschreven.

Hoofdstuk 2 is een kritische evaluatie van wat momenteel bekend en nog onbekend is over blaassensaties. Het hoofdstuk geeft aan dat het nodig is om terug te keren naar de basis principes omtrent blaassensaties en diagnostische middelen. We onderzoeken de mogelijkheid om met behulp van focus groepen belangrijke blaassensaties te identificeren en te evalueren welke gevoelens leiden tot de beslissing om te gaan plassen. We beargumenteren dat er verschillen zijn in wat kan worden omschreven als "introspectieve blaassensaties", zijnde sensaties die worden ervaren tijdens de blaasvulling, en "mictie 
sensaties", zijnde sensaties die direct voorafgaand aan een plas worden ervaren. Perifere informatie (informatie vanuit de blaas en de plasbuis) leidt tot deze "introspectieve sensaties" en "mictie sensaties". We beschrijven een alternatief model dat aangeeft hoe deze sensaties verschillend maar wel gerelateerd kunnen zijn. Bij het bestuderen van dergelijke ideeën en het aanstippen van de complexiteit van het onderwerp, proberen we het debat en aanvullend onderzoek op dit gebied te stimuleren. Hiermee beogen we uiteindelijk beter inzicht te krijgen in de fysiologie van blaassensaties en de pathologie van verhoogde aandrang (urgentie), vaker moeten plassen (frequentie) en ongewild urineverlies (incontinentie).

Hoofdstuk 3 evalueert de betrouwbaarheid van twee sensorische schalen (de mate van aandrang en de perceptie van blaasvulling). Ook de relatie tussen de beide schalen en het blaasvolume en de mogelijke verschillen in blaassensatie in een gecontroleerde omgeving (het ziekenhuis) versus een ongecontroleerde omgeving (thuis) worden beoordeeld. De resultaten tonen aan dat beide schalen betrouwbaar zijn en dat iedere schaal een significante relatie heeft met het blaasvolume. De overeenkomst tussen beide schalen en het geplaste volume is echter slecht. Dit betekent dat het gebruik van deze schalen, het daadwerkelijk meten van het geplaste volume, niet kan vervangen. Patiënten en vrijwilligers geven beide een hogere score aan hun blaasvulling en de mate van aandrang in de thuis omgeving ten opzichte van de ziekenhuis omgeving. Blaassensaties die patiënten ervaren in het ziekenhuis zijn daarom mogelijk een onderschatting van de symptomen die patiënten in hun dagelijks leven ervaren.

Hoofdstuk 4 introduceert een nieuwe niet invasieve techniek die kan worden gebruikt om het ontstaan en de ontwikkeling van blaassensaties te evalueren. Eveneens wordt de reproduceerbaarheid van deze methode getest. Door middel van een constante hoge diurese (urineproductie) worden blaassensaties geëvalueerd op een lege grafiek met de tijd op de $x$-as en de intensiteit van het gevoel op de y-as. Deze techniek werkt met een zogenaamd "water load protocol" waarbij in korte tijd een vaste grote hoeveelheid water moet worden gedronken. De resultaten van deze techniek tonen aan dat de urineproductie niet verschillend was op de verschillende meetmomenten. Er werden twee patronen van ontwikkeling van blaassensatie gevonden (introspectiecurves). In beide type patronen komt fase 1 overeen met de "stille fase". Fase 2 toont een langzame stijging in de intensiteit van het gevoel en in fase 3 treedt een snelle stijging in intensiteit op. Bij het eerste type leidt deze snelle stijging tot het gevoel van "moeten plassen". Bij het tweede type is er een vierde fase aantoonbaar waarbij de intensiteit van het gevoel wederom langzamer toeneemt en vervolgens leidt tot het gevoel van "moeten plassen". Per indivi$\mathrm{du}$ is het ontwikkelingspatroon gedurende de drie onderzoeksessies constant.

Hoofdstuk 5 beschrijft blaassensaties die worden ervaren door gezonde vrijwilligers tijdens een niet invasieve snelle blaasvulling in een gecontroleerde setting door middel van focus groep onderzoek. Om een constante maximale urineproductie te bewerkstelligen werd een "water load protocol" aan alle deelnemers gegeven. Gezonde vrijwilligers beschrijven twee soorten blaassensaties: (1) een druk, dit is het belangrijkste gevoel en is continu aanwezig en (2) een tintelend gevoel, dat niet door iedereen wordt ervaren en 
niet perse continu aanwezig is. Het gevoel van "moeten plassen" ontwikkelt zich in het volgende patroon, dat wordt beschreven door de termen: geen sensatie, zwakke gewaarwording, sterkere gewaarwording, zwakke aandrang, sterkere aandrang en "moeten plassen".

Hoofdstuk 6 beschrijft de terminologie en het patroon van blaassensaties dat wordt ervaren door patiënten met $\mathrm{OAB}$ tijdens een niet invasieve blaasvulling in een gecontroleerde setting. Deze resultaten worden vergeleken met de resultaten beschreven in hoofdstuk 5. In vergelijking met gezonde vrijwilligers, beschrijven patiënten hun blaasgevoelens ook als een druk of een tintelend gevoel. Het patroon van sensaties wordt beschreven met termen variërend van "geen gevoel" tot "een gevoel van te moeten plassen“. Er lijkt sprake te zijn van twee soorten aandrang: een plotselinge onhoudbare aandrang en een langzaam progressieve onhoudbare aandrang. De gemiddelde ontwikkeling van blaasgevoelens is significant verschillend tussen vrijwilligers en patiënten. Bovendien hebben patiënten een significant minder snelle urine productie. Mogelijk is dit het gevolg van een vochttekort door langdurig aanpassen van de vochtintake om minder vaak naar het toilet te hoeven gaan.

Hoofdstuk 7 is een algemene discussie die alle resultaten en discussies van de artikelen (hoofdstukken 2 tot en met 6) combineert met de gegevens van recente studies van andere auteurs en onderzoeksgroepen.

\section{Algemene conclusie}

Door middel van focus groep onderzoek en constante focus op de blaas, is het mogelijk om blaassensaties te beschrijven en te komen tot een terminologie die begrijpelijk en toepasbaar is voor gezonde vrijwilligers en patiënten met een overactieve blaas. Een "water load protocol" induceert een constante hoge urineproductie (diurese). Wanneer dit protocol wordt gecombineerd met constante focus op de blaas, ontstaat er een manier om het ontstaan van het gevoel "moeten plassen" op een niet invasieve wijze te evalueren. Naast het gebruik van focus groepen en de introspectiecurve, kunnen blaasgevoelens ook worden geëvalueerd door middel van een sensatie gerelateerd plasdagboek met daarop een schaal voor de mate van aandrang en de mate van blaasvolheid.

Om bovenstaande technieken te valideren en te kunnen gebruiken in de dagelijkse praktijk, moeten toekomstige studies bevestigen dat de introspectiecurve onafhankelijk is van de urineproductie. Daarnaast moeten we weten wat de precieze invloed van houding en locatie is op de ervaren blaasgevoelens. 

Curriculum Vitae and list of publications 


\section{Curriculum Vitae}

Rhea Heeringa werd geboren op 11 juli 1984 te Maastricht. In juni 2002 behaalde zij haar VWO diploma aan het Euro College te Maastricht. Vanaf september 2002 studeerde zij Geneeskunde aan de Universiteit van Maastricht. Haar artsexamen behaalde ze 6 jaar later in juni 2008. Overtuigd van het feit dat ze ooit wilde promoveren, begon ze, na vier maanden te hebben gewerkt als ANIOS urologie te Sittard, in januari 2009 met haar promotieonderzoek naar blaassensaties. Na 2 jaar fulltime onderzoek te hebben verricht, combineerde ze vanaf januari 2011 het afronden van haar thesis met een baan als ANIOS urologie in het Maastricht Universitair Medisch Centrum. Het onderzoek heeft geresulteerd in het proefschrift dat voor $u$ ligt. Na zich eerst te hebben gericht op urologie, heeft Rhea besloten een andere kant op te gaan. Nadat ze een ruim half jaar heeft gewerkt als ANIOS ouderen geneeskunde, is ze in september 2012 gestart met de huisartsopleiding aan de Universiteit van Maastricht.

\section{List of publications}

\section{Articles}

- Carotisendarteriëctomie: lange termijn resultaten in een nietopleidingsziekenhuis; Mevr. drs. R. Heeringa, dhr. Drs. F.TH.P.M. v.d. Linden, dhr. Dr. B. Winkens; Nederlands Tijdschrift voor Heelkunde jaargang 18, nummer 5, juli 2009

- On the nature of bladder sensation: the concept of sensory modulation; de Wachter, Heeringa, van Koeveringe, Gillespie; Neurourology and Urodynamics - 30 (7): 1220-6, 2011;Epub: 2011 June 9

- Normal bladder sensations in healthy volunteers: a focus group investigation; Heeringa, de Wachter, van Kerrebroeck, van Koeveringe; Neurourology and Urodynamics - 30 (7): 1350-5, 2012; Epub 2011 May 23

- Degree of urge, perception of bladder fullness and bladder volume: how are they related?; Heeringa, van Koeveringe, Winkens, van Kerrebroeck, de Wachter; Journal of Urology - 186 (4) : 1352-7, 2011;Epub: 2011 August 18

- Blaassensatie; R. Heeringa en G. van Koeveringe; Nederlands Tijdschrift voor Urologie nummer 2, april 2011

- Do patients with $O A B$ experience bladder sensations in the same way as healthy volunteers? A focus group investigation; Heeringa, van Koeveringe, Winkens, van Kerrebroeck, de Wachter; Neurourology and Urodynamics - 31 (4) : 521-5, 2012; Epub: 2012 March 6

- 'Focused introspection' during naturally increased diuresis: description and repeatability of a new method to study bladder sensation non-invasively; Heeringa, de Wachter, van Koeveringe, Winkens, van Kerrebroeck, Gillespie; Neurourology and Urodynamics, Submitted 


\section{Abstracts}

- How do naïve healthy subjects describe bladder sensations during a physiological bladder filling?; Heeringa, de Wachter, van Kerrebroeck, Gillespie, van Koeveringe; non-discussion poster ICS Toronto 2010

- $\quad$ Forced diuresis as a tool for the non-invasive evaluation of bladder sensation?; Heeringa, van Koeveringe, van Kerrebroeck, Gillespie, de Wachter; discussion poster ICS Toronto 2010

- Waterload protocol als middel om blaassensaties niet invasief te evalueren.; Heeringa, van Koeveringe, van Kerrebroeck, de Wachter; Presentatie NVU najaarsvergadering 2010

- Differences in bladder sensation between healthy volunteers and patients with overactive bladder symptoms.; Heeringa R, van Koeveringe $G$, van Kerrebroeck $\mathrm{P}$, de Wachter S; read by title ICS Glasgow 2011

- Degree of urge, perception of bladder fullness and bladder volume: how are they related?; Heeringa $R$, van Koeveringe $G$, van Kerrebroeck $P$, de Wachter S; read by title ICS Glasgow 2011 

Dankwoord 


\section{Dankwoord}

Veel mensen vroegen mij tijdens mijn promotietraject regelmatig wat onderzoek doen inhield. Ik hoop dat deze thesis daarin wat duidelijkheid schept en aantoont dat ik de afgelopen jaren niet stil heb gezeten. Brainstormen, artikelen zoeken en lezen, protocollen schrijven, data verzamelen en analyseren en natuurlijk artikelen schrijven, niets van dat alles is mij nog onbekend. Ondanks dat ik aan zelfmotivatie en discipline geen gebrek heb, heeft het verrichten van mijn onderzoek zijn pieken en dalen gekend. Pieken die ik heb kunnen vieren met collega's met name in de vorm van een extra koffie break. Dalen waarbij ik werd opgepept door collega's met name in de vorm van, hoe kan het ook anders, een extra koffie break. Dit geeft direct aan dat mijn thesis niet alleen van mijn hand komt. Ik ben veel mensen, die direct of indirect hebben geholpen, dank verschuldigd.

Dr. van Koeveringe, beste Gommert,

Allereerst wil ik je bedanken voor je vertrouwen in mijn vaardigheden en je steun tijdens afgelopen jaren. Je reactie op het nieuws dat ik wilde stoppen met urologie was onverwacht positief. Ik heb niet alleen vakinhoudelijk veel van je geleerd, maar ook op persoonlijk gebied. Zo heb ik onder andere door jouw drukke agenda tegenwoordig (iets) meer geduld. Ook wil ik je bedanken voor de mogelijkheden die je me hebt geboden, met name om, tot op bepaalde hoogte, zelf de richting binnen mijn onderzoeken te bepalen.

Professor dr. de Wachter, beste Stefan,

Wat vond ik ze vervelend al die vragen waar ik niet direct een antwoord op had, maar wat heb ik er veel van geleerd. Op momenten dat ik het even niet meer zag zitten, wist je me weer te herinneren aan de waarde van alle informatie die we afgelopen jaren hebben verzameld. Bedankt voor alle stimulerende en motiverende discussies. Hoewel het me soms niet snel genoeg ging, denk aan het repeatability artikel, zou zonder jouw hulp deze thesis nu niet in deze vorm voor ons liggen. Bedankt voor al jouw tijd.

\section{Professor dr. van Kerrebroeck,}

Bedankt voor de mogelijkheid om promotie onderzoek te mogen verrichten. $U$ stond achter de schermen altijd klaar om advies te geven. Hoewel we het niet altijd met elkaar eens waren, waren we het er wel over eens dat deze onderzoeken de moeite waard zijn en de thesis af zou moeten worden gemaakt. Dat is ons gelukt.

Mijn collega-onderzoekers wil ik bedanken voor alle adviezen en hulp bij mijn onderzoeken. Maar met name wil ik jullie bedanken voor onze koffie breaks, quizavonden, feestjes en etentjes. Kortom bedankt voor alle gezelligheid. Tom, Martijn, Randall en Vera, ondanks dat we niet meer allemaal binnen de urologie werken, hoop ik dat onze vriendschap blijft en we nog veel samen zullen ondernemen. 
Professor dr. Gillespie, dear James,

You always believed that my research was worth doing. Thank you for your advice and all your splendid ideas. I especially appreciate the time you took for me in Toronto before my presentation in order to make me feel at ease.

Ook ben ik grote dank verschuldigd aan alle patiënten en gezonde vrijwilligers, die op vrijwillige basis liters water hebben gedronken en dagen in een maatbeker hebben geplast. Bedankt voor jullie deelname en inzet.

Alle medewerkers van het secretariaat, de poli en de functie afdeling urologie wil ik bedanken voor hun medewerking en de "bijklets-momenten" tussendoor.

Familie en vrienden. Hoewel niet iedereen van jullie altijd begreep waar ik mee bezig was, waren jullie altijd geïnteresseerd in mijn vorderingen. Dank hiervoor.

Nienke en Anne, bedankt dat jullie mijn paranymfen willen zijn. We hebben het helaas allemaal erg druk, maar op momenten dat ik jullie zie is het als vanouds. Nienke, misschien de volgende bevalling dan maar? Anne, helaas verhuis je naar de andere kant van het land (zo klinkt het wel erg dramatisch hè), maar ik kom je daar graag opzoeken.

Lyanne en Kirsten, wat fijn dat jullie niet alleen mijn zusjes maar ook mijn vriendinnen zijn.

Pap en mam. Wat heb ik toch een geluk om uit zo'n warm nest te komen. Jullie hebben altijd in mij geloofd en me gesteund in al mijn keuzes. De laatste jaren ben ik onze gezamenlijke uitjes steeds meer gaan waarderen. Bedankt voor jullie onvoorwaardelijke liefde.

Lieve Jelmer, jou ben ik veel dank verschuldigd. Je hebt afgelopen jaren niet alleen alle vreugde maar ook alle stress van dichtbij meegemaakt. Gelukkig heb jij zeeën van geduld en ken je me door en door. Met jou kan ik de wereld aan!

Lieve Doutzen, jij hebt mijn leven verrijkt. Ik hoop dat je net zo gelukkig wordt met mij als dat ik met jou ben.

$\mathrm{Nu}$ is het dan zover, we zetten er een punt achter 